\title{
Supporting Information (JA0365330)
}

\section{The First Total Synthesis of Discorhabdin A}

By Hirofumi Tohma, Yu Harayama, Miki Hashizume, Minako Iwata, Yorito Kiyono, Masahiro Egi, and Yasuyuki Kita*

Complete experimental procedures and characterization data for all previously unreported compounds described herein, including chiral HPLC data for 26 


\section{Experimental Section}

General. Melting points were determined on a Yanagimoto Micro Melting Point Apparatus and are uncorrected. Infrared (IR) absorption spectra were recorded as $\mathrm{KBr}$ pellets on a SHIMADZU FTIR-8400 spectrophotometer. NMR spectra were measured on a JEOL JMN-EX 270 or JEOL JNM-AL 300 or JEOL JNM-GSX 500. NMR spectra were obtained in $\mathrm{CDCl}_{3}$ except where noted with TMS as an internal standard. Mass spectra (MS) were measured on a JEOL JMS-AMII50 or JEOL JMS-D300 instrument. E. Merck silica gel 60 for column chromatography and E. Merck precoated TLC plates, silica gel $\mathrm{F}_{254}$ for preparative thin layer chromatography were used. Organic layers were dried with anhydrous $\mathrm{MgSO}_{4}$ or $\mathrm{Na}_{2} \mathrm{SO}_{4}$. PIFA and iodosobenzene are commercially available. All reagents were of reagent grade, unless otherwise stated. In general, reactions were carried out in anhydrous solvents, unless otherwise mentioned.

\section{2-(6-Hydroxy-1-oxo-2,3-dihydro-1H-1 $\lambda$-benzo[b]thiophen-2-ylamino)-[1,4]- naphthoquinone $(5 b)$.}

To a stirred solution of 2-[6-tert-butyldimethylsilanyloxy]-2,3-dihydrobenzo[b]thiophen-2-ylamino] [1,4]naphthoquinone 5a $(4.7 \mathrm{mg}, 0.0145 \mathrm{mmol})$ in anhydrous $\mathrm{CH}_{2} \mathrm{Cl}_{2}(1.0 \mathrm{~mL})$ was added $m$-chloroperbenzoic acid (mCPBA) $(3.7 \mathrm{mg}, 0.0145 \mathrm{mmol})$ at $0^{\circ} \mathrm{C}$ under a nitrogen atmosphere. The mixture was stirred for $45 \mathrm{~min}$ at $0^{\circ} \mathrm{C}$ and then quenched with aqueous saturated $\mathrm{Na}_{2} \mathrm{~S}_{2} \mathrm{O}_{3}$ and extracted with AcOEt. The combined organics were dried and concentrated in vacuo. The residue was purified by flash chromatography $\left(\mathrm{CH}_{2} \mathrm{Cl}_{2} / \mathrm{MeOH}=20 / 1\right)$ to give $\mathbf{5 b}(2.2 \mathrm{mg}, 45 \%)$ as a yellow solid.

5a: a brown solid. mp 225-227 ${ }^{\circ} \mathrm{C}$. IR (KBr): v 3000-3500, 2922, 2851, 1676, 1603 , 1572, $1502 \mathrm{~cm}^{-1} .{ }^{1} \mathrm{H}$ NMR (300 MHz): $\delta 3.02$ (br s, $\left.1 \mathrm{H}\right), 3.31$ (dd, $\left.1 \mathrm{H}, J=16.0,2.0 \mathrm{~Hz}\right)$, $3.65(\mathrm{dd}, 1 \mathrm{H}, J=15.5,1.0 \mathrm{~Hz}), 5.37$ (t, 1H, $J=8.5 \mathrm{~Hz}), 5.83(\mathrm{~s}, 1 \mathrm{H}), 6.30(\mathrm{~d}, 1 \mathrm{H}, J=$ $9.0 \mathrm{~Hz}), 6.57$ (d, 1H, $J=7.5 \mathrm{~Hz}), 6.71$ (br s, 1H), 7.12 (d, 1H, $J=8.5 \mathrm{~Hz}), 7.61$ (t, 1H, $J$ $=8.5,6.5 \mathrm{~Hz}), 7.72(\mathrm{t}, 1 \mathrm{H}, J=7.5,6.5 \mathrm{~Hz}), 8.01(\mathrm{~d}, 1 \mathrm{H}, J=8.0 \mathrm{~Hz}), 8.09(\mathrm{~d}, 1 \mathrm{H}, J=$ 8.0 Hz). ${ }^{13} \mathrm{C}$ NMR (75 MHz): $\delta 41.8,62.5,104.1,110.0,112.3,125.9,126.3,126.4$, 127.9, 130.4, 132.4, 133.1, 134.9, 140.3, 145.8, 156.1, 181.3, 183.2. HRMS calcd for 
$\mathrm{C}_{20} \mathrm{H}_{17} \mathrm{NO}_{3}\left(\mathrm{M}^{+}\right)$323.0616, found 323.0612.

5b: IR (KBr): v 3500-2700, 1678, 1607, 1574, $1493 \mathrm{~cm}^{-1} .{ }^{1} \mathrm{H}$ NMR (300 MHz, $\left.\mathrm{CD}_{3} \mathrm{OD}\right): \delta 3.67(\mathrm{dd}, 1 \mathrm{H}, J=16.2,6.9 \mathrm{~Hz}), 3.86(\mathrm{dd}, 1 \mathrm{H}, J=15.9,9.0 \mathrm{~Hz}), 5.34(\mathrm{t}, 1 \mathrm{H}$, $J=7.2 \mathrm{~Hz}), 6.25(\mathrm{~s}, 1 \mathrm{H}), 7.08(\mathrm{dd}, 1 \mathrm{H}, J=8.4,2.4 \mathrm{~Hz}), 7.31(\mathrm{~d}, 1 \mathrm{H}, J=2.4 \mathrm{~Hz}), 7.43$ $(\mathrm{d}, 1 \mathrm{H}, J=8.4 \mathrm{~Hz}), 7.76(\mathrm{td}, 1 \mathrm{H}, J=7.5,1.5 \mathrm{~Hz}), 7.83(\mathrm{td}, 1 \mathrm{H}, J=7.5,1.5 \mathrm{~Hz}), 8.07$ $(\mathrm{dd}, 1 \mathrm{H}, J=7.5,1.2 \mathrm{~Hz}), 8.12(\mathrm{dd}, 1 \mathrm{H}, J=7.5,1.2 \mathrm{~Hz})$. HR-FABMS calcd for $\mathrm{C}_{18} \mathrm{H}_{13} \mathrm{NO}_{4} \mathrm{SNa}\left(\mathrm{M}+\mathrm{Na}^{+}\right)$; 362.0463, found 362.0449. This compound gradually decomposed during ${ }^{13} \mathrm{C}$ NMR measurement due to its unstability.

\section{2-(1,4-Dioxo-1,4-dihydronaphthalen-2-ylamino)-3-(4-hydroxyphenyl)propionic} acid methyl ester (7).

To a stirred solution of $6(1.05 \mathrm{~g}, 5.40 \mathrm{mmol})$ in $\mathrm{MeOH}(8.0 \mathrm{~mL})$ was added 1,4naphthoquinone ( $854 \mathrm{mg}, 5.40 \mathrm{mmol}$ ) at room temperature, and the mixture was stirred overnight (for $12 \mathrm{~h}$ ) under the same conditions. The resulting mixture was concentrated in vacuo, then the residue was purified by column chromatography ( $n$ hexane/AcOEt=3:1) to give $7(1.08 \mathrm{~g}, 57 \%)$ as a brown solid.

7: mp 70-72 ${ }^{\circ} \mathrm{C}$ (from EtOH). IR (KBr): v 3354, 1746, 1678, 1607, 1568, $1514 \mathrm{~cm}^{-1}$. ${ }^{1} \mathrm{H}$ NMR (300 MHz): $\delta 3.07(\mathrm{dd}, 1 \mathrm{H}, J=14.0,6.5 \mathrm{~Hz}), 3.17(\mathrm{dd}, 1 \mathrm{H}, J=14.0,5.5 \mathrm{~Hz})$, $3.74(\mathrm{~s}, 3 \mathrm{H}), 4.21-4.30(\mathrm{~m}, 1 \mathrm{H}), 5.66(\mathrm{~s}, 1 \mathrm{H}), 6.14(\mathrm{~s}, 1 \mathrm{H}), 6.33(\mathrm{~d}, 1 \mathrm{H}, J=8.0 \mathrm{~Hz}), 6.77$ $(\mathrm{d}, 2 \mathrm{H}, J=8.5 \mathrm{~Hz}), 6.98(\mathrm{~d}, 2 \mathrm{H}, J=8.5 \mathrm{~Hz}), 7.61(\mathrm{t}, 1 \mathrm{H}, J=7.5 \mathrm{~Hz}), 7.71(\mathrm{t}, 1 \mathrm{H}, J=$ $7.5 \mathrm{~Hz}), 8.03(\mathrm{~d}, 1 \mathrm{H}, J=7.6 \mathrm{~Hz}), 8.07(\mathrm{~d}, 1 \mathrm{H}, J=7.6 \mathrm{~Hz}) .{ }^{13} \mathrm{C} \mathrm{NMR}(75 \mathrm{MHz}): \delta 36.7$, 52.7, 56.2, 101.9, 115.9, 126.2, 126.5, 126.6, 130.3, 130.4, 132.3, 133.2, 134.8, 146.8, 155.4, 170.8, 181.1, 183.5. HRMS calcd for $\mathrm{C}_{20} \mathrm{H}_{17} \mathrm{NO}_{3}\left(\mathrm{M}^{+}\right)$351.1106, found 351.1116.

\section{1,2,3,4,5,10-Hexahydrobenzo[6,7-g]quinoline-5,10-dione-2-carboxyl-4-spiro4'-} cyclohexa-2',5'-dien-1'-one (8).

To a stirred solution of $6(53.8 \mathrm{mg}, 0.153 \mathrm{mmol})$ in $\mathrm{CF}_{3} \mathrm{CH}_{2} \mathrm{OH}(5.0 \mathrm{~mL})$ was added MK10 (33.6 mg) and PIFA $(98.8 \mathrm{mg}, 0.230 \mathrm{mmol})$ sequentially at room temperature under a nitrogen atmosphere. The mixture was stirred for $30 \mathrm{~min}$ and then concentrated 
in vacuo. The residue was purified by column chromatography ( $n$-hexane/AcOEt=1:1) to give 1,2,3,4,5,10-hexahydrobenzo[6,7-g]quinoline-5,10-dione-2-methoxycarbonyl-4spiro4'-cyclohexa-2', 5'-dien-1'-one (22.5 mg, $42 \%)$ as a red solid.

mp 93-96 ${ }^{\circ} \mathrm{C}$ (from AcOEt). IR (KBr): v 1745, 1661, 1601, 1570, 1504cm ${ }^{-1} .{ }^{1} \mathrm{H}$ NMR (300 MHz): $\delta$ 2.03-2.21 (m, 2H), 3.85 (s, 3H), 4.28 (dd, 1H, $J=10.0,4.0 \mathrm{~Hz}), 6.39$ (d, $1 \mathrm{H}, J=10.0 \mathrm{~Hz}), 6.43(\mathrm{~d}, 1 \mathrm{H}, J=10.0 \mathrm{~Hz}), 6.73(\mathrm{~s}, 1 \mathrm{H}), 6.85(\mathrm{dd}, 1 \mathrm{H}, J=10.0,3.0 \mathrm{~Hz})$, $6.92(\mathrm{dd}, 1 \mathrm{H}, J=10.0,3.0 \mathrm{~Hz}), 7.61(\mathrm{t}, 1 \mathrm{H}, J=7.5 \mathrm{~Hz}), 7.71(\mathrm{t}, 1 \mathrm{H}, J=7.5 \mathrm{~Hz}), 7.99$ (d, $1 \mathrm{H}, J=7.5 \mathrm{~Hz}), 8.04(\mathrm{~d}, 1 \mathrm{H}, J=7.0 \mathrm{~Hz}) \cdot{ }^{13} \mathrm{C}$ NMR $(75 \mathrm{MHz}): \delta 36.5,40.0,50.2,53.2$, 110.2, 126.1, 126.5, 128.1, 129.0, 129.8, 132.3, 133.2, 135.2, 144.0, 149.8, 153.6, 170.2, 179.7, 180.1, 185.2. HRMS calcd for $\mathrm{C}_{20} \mathrm{H}_{13} \mathrm{NO}_{3}\left(\mathrm{M}^{+}\right)$349.0950, found 349.0965 .

To a stirred solution of 1,2,3,4,5,10-hexahydrobenzo[6,7-g]quinoline-5,10-dione-2methoxycarbonyl-4-spiro4'-cyclohexa-2',5'-dien-1'-one (18.1 mg, $0.0518 \mathrm{mmol})$ in 1,4-dioxane $(1.5 \mathrm{~mL})$ was added $6 \mathrm{~N} \mathrm{HCl}(1.5 \mathrm{~mL})$ at room temperature. The mixture was stirred at $60^{\circ} \mathrm{C}$ for $2.5 \mathrm{~h}$, then cooled to room temperature, and extracted with AcOEt. The combined organic layer was washed with brine, dried, and evaporated in vacuo. The residue was purified by column chromatography $(\mathrm{AcOEt} / \mathrm{AcOH}=100: 1)$ to give $8(13.2 \mathrm{mg}, 76 \%)$ as an orange solid.

8: $\mathrm{mp} 205-207^{\circ} \mathrm{C}$. IR (KBr): v 2873, 1736, 1657, 1599, 1568, $1502 \mathrm{~cm}^{-1} .{ }^{1} \mathrm{H}$ NMR $\left(500 \mathrm{MHz}, \mathrm{CD}_{3} \mathrm{OD}\right): \delta 2.16(\mathrm{~d}, 2 \mathrm{H}, J=6.5 \mathrm{~Hz}), 4.38(\mathrm{t}, 1 \mathrm{H}, J=6.0 \mathrm{~Hz}), 6.32(\mathrm{~d}, 2 \mathrm{H}, J$ $=10.0 \mathrm{~Hz}), 7.04(\mathrm{dd}, 1 \mathrm{H}, J=10.0,2.5 \mathrm{~Hz}), 7.17(\mathrm{dd}, 1 \mathrm{H}, J=10.0,2.5 \mathrm{~Hz}), 7.66(\mathrm{td}, 1 \mathrm{H}$, $J=7.5,1.0 \mathrm{~Hz}), 7.73(\mathrm{td}, 1 \mathrm{H}, J=7.5,1.0 \mathrm{~Hz}), 7.90$ (d, 1H, $J=7.0 \mathrm{~Hz}), 8.04$ (d, 1H, $J=$ $6.5 \mathrm{~Hz}) .{ }^{13} \mathrm{C}$ NMR (75 MHz, $\left.\mathrm{CD}_{3} \mathrm{OD}\right): \delta 37.3,42.0,51.5,109.7,127.1,128.4,128.6$, 131.6, 133.4, 134.9, 136.1, 146.7, 154.9, 157.0, 181.0, 181.4, 188.1. HRMS calcd for $\mathrm{C}_{19} \mathrm{H}_{14} \mathrm{NO}_{3}\left(\mathrm{M}^{+}\right)$336.0872, found 332.0875 .

\section{1,2,3,4,5,10-Hexahydrobenzo[6,7-g]quinoline-5,10-dione-2-methoxy-4-spiro4'-} cyclohexa-2',5'-dien-1'-one (10). 
A solution of $8(7.7 \mathrm{mg}, 0.023 \mathrm{mmol})$ in $\mathrm{MeOH}(5.0 \mathrm{~mL})$ containing $\mathrm{NaOMe}(0.062$ $\mathrm{mg}, 1.15 \times 10^{-3} \mathrm{mmol}$ ) was electrolyzed at room temperature using a $6.4 \mathrm{~cm}^{2}$ of graphite anode-graphite cathode system in a non-divided cell under a nitrogen atmosphere. The electrolysis current was maintained at $1.0 \mathrm{~mA}$ during the electrolysis. After the theoretical amount of electricity had passed (for $74 \mathrm{~min}$ ), the electrolyzed solution was evaporated to dryness in vacuo. The residue was purified by column chromatography ( $n$-hexane/AcOEt $=1: 1)$ to give $\mathbf{1 0}(4.4 \mathrm{mg}, 60 \%)$ as a yellow solid

10: $\mathrm{mp} 192-195{ }^{\circ} \mathrm{C}$ (from AcOEt). IR (KBr): $v$ 3360, 1660, 1605, 1575, $1505 \mathrm{~cm}^{-1}$. ${ }^{1} \mathrm{H}$ NMR (300 MHz): $\delta 2.00(\mathrm{~d}, 1 \mathrm{H}, J=14.0 \mathrm{~Hz}), 2.09(\mathrm{dd}, 1 \mathrm{H}, J=14.0,3.5 \mathrm{~Hz}), 3.42$ (s, 3H), 4.84-4.88 (m, 1H), $6.31(\mathrm{dd}, 1 \mathrm{H}, J=10.0,1.8 \mathrm{~Hz}), 6.43(\mathrm{dd}, 1 \mathrm{H}, J=10.0,1.8$ $\mathrm{Hz}), 6.84(\mathrm{dd}, 1 \mathrm{H}, J=10.0,3.0 \mathrm{~Hz}), 6.90(\mathrm{br} \mathrm{s}, 1 \mathrm{H}), 7.25(\mathrm{dd}, 1 \mathrm{H}, J=10.0,3.0 \mathrm{~Hz})$, $7.63(\mathrm{td}, 1 \mathrm{H}, J=7.6,1.2 \mathrm{~Hz}), 7.72(\mathrm{td}, 1 \mathrm{H}, J=7.6,1.2 \mathrm{~Hz}), 8.02(\mathrm{~d}, 1 \mathrm{H}, J=7.5 \mathrm{~Hz})$, $8.05(\mathrm{~d}, 1 \mathrm{H}, J=7.5 \mathrm{~Hz}) .{ }^{13} \mathrm{C} \mathrm{NMR}(67 \mathrm{MHz}): \delta 38.1,39.3,55.2,79.3,112.3,126.0$, $126.5,127.6,127.8,129.8,132.3,132.9,135.1,142.5,151.8,154.1,180.2,185.6$. HRMS calcd for $\mathrm{C}_{19} \mathrm{H}_{13} \mathrm{NO}_{4}\left(\mathrm{M}^{+}\right)$321.1001, found 321.1018.

\section{2-\{1-(tert-Butyldimethylsilanyloxymethyl)-2-[4-(tert-butyldimethyl-}

\section{silanyloxy)phenyl)ethylamino\}[1,4]naphthoquinone $(11)$.}

A solution of $7(203 \mathrm{mg}, 0.578 \mathrm{mmol})$ in anhydrous THF $(10 \mathrm{~mL})$ was added to a stirred suspension of $\mathrm{LiBH}_{4}(57.0 \mathrm{mg}, 2.62 \mathrm{mmol})$ in anhydrous THF $(5.0 \mathrm{~mL})$ at $0^{\circ} \mathrm{C}$ under a nitrogen atmosphere. The mixture was stirred for $2 \mathrm{~h}$ at room temperature and then quenched with THF saturated with water at $0^{\circ} \mathrm{C}$ and extracted with AcOEt. The combined organics were washed with brine, dried and concentrated in vacuo. The residue was purified by flash chromatography ( $n$-hexane/AcOEt=1/1) to give 2-[1hydroxymethyl-2-(4-hydroxyphenyl)ethylamino][1,4]naphthoquinone (187 mg, quant) as a red crystal.

mp 167-169 ${ }^{\circ} \mathrm{C}$. IR (KBr): v 3345, 2500, 1680, 1600, 1570, $1515 \mathrm{~cm}^{-1} .{ }^{1} \mathrm{H}$ NMR $(270$ $\left.\mathrm{MHz}, \mathrm{CD}_{3} \mathrm{OD}\right): \delta 2.78(\mathrm{dd}, 1 \mathrm{H}, J=14.5,8.0 \mathrm{~Hz}), 2.89(\mathrm{dd}, 1 \mathrm{H}, J=14.0,6.5 \mathrm{~Hz}), 3.62-$ $3.70(\mathrm{~m}, 3 \mathrm{H}), 5.66(\mathrm{~s}, 1 \mathrm{H}), 5.67(\mathrm{~s}, 1 \mathrm{H}), 6.68(\mathrm{~d}, 2 \mathrm{H}, J=8.5 \mathrm{~Hz}), 7.07(\mathrm{~d}, 2 \mathrm{H}, J=8.5$ 
$\mathrm{Hz}), 7.65(\mathrm{td}, 1 \mathrm{H}, J=7.5,1.5 \mathrm{~Hz}), 7.75(\mathrm{td}, 1 \mathrm{H}, J=7.5,1.5 \mathrm{~Hz}), 7.97$ (d, 1H, $J=7.5$ $\mathrm{Hz}), 8.02$ (d, $1 \mathrm{H}, J=7.5 \mathrm{~Hz}) .{ }^{13} \mathrm{C} \mathrm{NMR}\left(67 \mathrm{MHz}, \mathrm{CD}_{3} \mathrm{OD}\right): \delta 36.8,57.5,63.3,100.4$, 116.2, 126.6, 127.2, 129.6, 131.2, 131.8, 133.2, 134.6, 135.7, 150.1, 157.0, 182.2, 184.6. HR-FABMS calcd for $\mathrm{C}_{19} \mathrm{H}_{13} \mathrm{NO}_{4}\left(\mathrm{M}+\mathrm{H}^{+}\right) ; 324.1236$, found 324.1232.

To a stirred solution of 2-[1-hydroxymethyl-2-(4-hydroxyphenyl)ethylamino]$[1,4]$ naphthoquinone $(3.37 \mathrm{~g}, 10.4 \mathrm{mmol})$ in anhydrous $\mathrm{CH}_{2} \mathrm{Cl}_{2}(150 \mathrm{~mL})$ was added tert-butyldimethylsilyl chloride (TBSCl) $\quad(4.71 \quad \mathrm{~g}, \quad 31.3 \mathrm{mmol})$ and 1,8diazabicyclo[5.4.0]undec-7-ene (DBU) $(7.8 \mathrm{ml}, 52.0 \mathrm{mmol})$ sequentially at $0^{\circ} \mathrm{C}$ under a nitrogen atmosphere. The mixture was stirred for $1 \mathrm{~h}$ at room temperature and then concentrated in vacuo. The residue was purified by flash chromatography ( $n$ hexane/AcOEt=30/1) to give $\mathbf{1 1}$ (5.75 g, quant) as an orange oil.

11: IR (KBr): v 2953, 2856, 1678, 1607, 1574, $1504 \mathrm{~cm}^{-1} .{ }^{1} \mathrm{H}$ NMR (500 MHz): $\delta$ $0.05(\mathrm{~s}, 6 \mathrm{H}), 0.14(\mathrm{~s}, 3 \mathrm{H}), 0.15(\mathrm{~s}, 3 \mathrm{H}), 0.93(\mathrm{~s}, 9 \mathrm{H}), 0.94(\mathrm{~s}, 9 \mathrm{H}), 2.85-2.87(\mathrm{~m}, 2 \mathrm{H})$, $3.56(\mathrm{~m}, 1 \mathrm{H}), 3.61(\mathrm{~d}, 2 \mathrm{H}, J=3.5 \mathrm{~Hz}), 5.75(\mathrm{~s}, 1 \mathrm{H}), 6.25(\mathrm{~d}, 1 \mathrm{H}, J=9.0 \mathrm{~Hz}), 6.75(\mathrm{~d}$, $2 \mathrm{H}, J=8.5 \mathrm{~Hz}), 7.05(\mathrm{~d}, 2 \mathrm{H}, J=8.0 \mathrm{~Hz}), 7.59(\mathrm{t}, 1 \mathrm{H}, J=7.5 \mathrm{~Hz}), 7.70(\mathrm{t}, 1 \mathrm{H}, J=7.5$ $\mathrm{Hz}), 8.02(\mathrm{~d}, 1 \mathrm{H}, J=7.5 \mathrm{~Hz}), 8.08(\mathrm{~d}, 1 \mathrm{H}, J=7.0 \mathrm{~Hz}) .{ }^{13} \mathrm{C} \mathrm{NMR}(125 \mathrm{MHz}): \delta-5.50$, $-5.42,-4.47,18.2,25.7,25.9,35.4,54.9,61.9,100.9,120.3,126.2,126.3,129.9,130.3$, 130.6, 131.9, 133.7, 134.7, 147.1, 154.5, 181.8, 183.0. HRMS calcd for $\mathrm{C}_{31} \mathrm{H}_{43} \mathrm{NO}_{4} \mathrm{Si}_{2}$ $\left(\mathrm{M}^{+}\right)$551.2887, found 551.2888.

\section{1,2,3,4,5,10-Hexahydrobenzo[6,7-g]quinoline-5,10-dione-2-hydroxymethyl-4-} spiro-4'-cyclohexa-2',5'-dien-1'-one (12).

To a stirred solution of 11 (52.9 $\mathrm{mg}, 0.0959 \mathrm{mmol})$ in $\mathrm{CF}_{3} \mathrm{CH}_{2} \mathrm{OH}(1.0 \mathrm{~mL})$ was added PIFA (49.5 mg, $0.115 \mathrm{mmol}$ ) at room temperature under a nitrogen atmosphere. The mixture was stirred for $0.5 \mathrm{~h}$ and then concentrated in vacuo. The residue was purified by flash chromatography ( $n$-hexane/AcOEt $=2 / 1)$ to give $1,2,3,4,5,10$ hexahydrobenzo[6,7-g]quinoline-5,10-dione-2-tert-butyl-dimethylsilyloxymethyl-4spiro-4'-cyclohexa-2',5'-dien-1'-one (29.7 mg, 71\%) as a red solid. 
mp 63-67 ${ }^{\circ} \mathrm{C}$. IR (KBr): v 2952, 2852, 1661, 1599, 1568, $1497 \mathrm{~cm}^{-1} .{ }^{1} \mathrm{H}$ NMR (500 MHz): $\delta 0.10$ (s, 3H), $0.12(\mathrm{~s}, 3 \mathrm{H}), 0.94(\mathrm{~s}, 9 \mathrm{H}), 1.61(\mathrm{dd}, 1 \mathrm{H}, J=12.5,2.0 \mathrm{~Hz}), 1.76$ $(\mathrm{dd}, 1 \mathrm{H}, J=13.0,11.5 \mathrm{~Hz}), 3.53(\mathrm{dd}, 1 \mathrm{H}, J=10.0,9.0 \mathrm{~Hz}), 3.64-3.69$ (m, 1H), 3.85 (dd, $1 \mathrm{H}, J=10.0,4.0 \mathrm{~Hz}), 6.31(\mathrm{~d}, 1 \mathrm{H}, J=9.5 \mathrm{~Hz}), 6.40$ (d, 1H, $J=9.0 \mathrm{~Hz}), 6.68$ (brs, 1H), 6.89-6.94 (m, 2H), $7.57(\mathrm{~d}, 1 \mathrm{H}, J=7.5 \mathrm{~Hz}), 7.67(\mathrm{~d}, 1 \mathrm{H}, J=7.5 \mathrm{~Hz}), 7.98(\mathrm{~d}, 1 \mathrm{H}, J=$ $8.5 \mathrm{~Hz}), 8.00(\mathrm{~d}, 1 \mathrm{H}, J=8.0 \mathrm{~Hz}) .{ }^{13} \mathrm{C} \mathrm{NMR}(125 \mathrm{MHz}): \delta-5.49,-5.37,18.2,25.8,36.6$, 40.2, 49.6, 65.7, 109.8, 125.9, 126.5, 127.3, 128.7, 130.0, 132.0, 133.6, 135.1, 144.8, 150.4, 155.4, 179.5, 180.6, 185.5. HRMS calcd for $\mathrm{C}_{23} \mathrm{H}_{29} \mathrm{NO}_{4} \mathrm{Si}\left(\mathrm{M}^{+}\right) 435.1866$, found 435.1872 .

To a stirred solution of 1,2,3,4,5,10-hexahydrobenzo[6,7-g]quinoline-5,10-dione-2tert-butyl-dimethylsilyloxymethyl-4-spiro-4'-cyclohexa-2',5'-dien-1'-one (2.0 g, 4.94 mmol) in THF $(100 \mathrm{~mL})-\mathrm{H}_{2} \mathrm{O}(1.0 \mathrm{~mL})$ was added tetra $n$-buthylammonium fluoride (TBAF) (1M solution in THF) $(5.0 \mathrm{~mL}, 5.0 \mathrm{mmol})$ at $0^{\circ} \mathrm{C}$ under a nitrogen atmosphere. The mixture was stirred for $1 \mathrm{~h}$ at room temperature and then quenched with aqueous saturated $\mathrm{NH}_{4} \mathrm{Cl}$ and extracted with AcOEt. The combined organics were washed with brine, dried and concentrated in vacuo. The residue was purified by flash chromatography ( $n$-hexane/AcOEt=1/2) to give $12(1.12 \mathrm{~g}, 71 \%)$ as an orange crystal.

12: $\mathrm{mp} 228-230{ }^{\circ} \mathrm{C}$. IR (KBr): $v$ 3380, 1675, 1600, 1570, 1505, $\mathrm{cm}^{-1} .{ }^{1} \mathrm{H}$ NMR $(300$ MHz): $\delta 1.65(\mathrm{~d}, 1 \mathrm{H}, J=11.5 \mathrm{~Hz}), 1.85(\mathrm{dd}, 1 \mathrm{H}, J=11.5,10.0 \mathrm{~Hz}), 3.61-3.77(\mathrm{~m}, 2 \mathrm{H})$, $3.95(\mathrm{dd}, 1 \mathrm{H}, J=10.0,3.0 \mathrm{~Hz}), 6.33(\mathrm{~d}, 1 \mathrm{H}, J=10.0 \mathrm{~Hz}), 6.42(\mathrm{~d}, 1 \mathrm{H}, J=10.0 \mathrm{~Hz})$, 6.69 (brs, 1H), 6.91-6.97 (m, 2H), 7.58 (t, 1H, $J=6.5 \mathrm{~Hz}), 7.69$ (t, $1 \mathrm{H}, J=6.5 \mathrm{~Hz}), 7.99$ $(\mathrm{d}, 1 \mathrm{H}, J=8.5 \mathrm{~Hz}), 8.02(\mathrm{~d}, 1 \mathrm{H}, J=8.0 \mathrm{~Hz}) .{ }^{13} \mathrm{C} \mathrm{NMR}(68 \mathrm{MHz}): \delta 36.5,40.3,49.6$, 65.0, 109.8, 126.0, 126.3, 127.2, 128.6, 129.8, 132.0, 133.3, 135.1, 144.9, 150.6, 155.6, 179.5, 180.3, 185.5. HR-FABMS calcd for $\mathrm{C}_{19} \mathrm{H}_{16} \mathrm{NO}_{4}\left(\mathrm{M}+\mathrm{H}^{+}\right) ; 322.1079$, found 322.1079 .

\section{1,2,3,4,5,10-Hexahydrobenzo[6,7-g]quinoline-5,10-dione-2-methoxy-4-spiro-4'- cyclohexa-2',5'-dien-1'-one (10).}


To a stirred solution of $\mathbf{1 2}(81.3 \mathrm{mg}, 0.253 \mathrm{mmol})$ in anhydrous $\mathrm{CH}_{2} \mathrm{Cl}_{2}(8.0 \mathrm{~mL})-$ $\mathrm{MeOH}(4.0 \mathrm{~mL})$ was added $\mathrm{Pb}(\mathrm{OAc})_{4}(448.7 \mathrm{mg}, 1.01 \mathrm{mmol})$ at room temperature under a nitrogen atmosphere. The mixture was stirred for $5 \mathrm{~h}$ and then concentrated in vacuo. The residue was purified by flash chromatography ( $n$-hexane/AcOEt $=1 / 1)$ to give $10(49.5 \mathrm{mg}, 61 \%)$ as a yellow solid.

\section{1,2,3,4,5,10-Hexahydrobenzo[6,7-g]quinoline-5,10-dione-2-acetylthio-4-spiro4'- cyclohexa-2',5'-dien-1'-one (9a).}

To a stirred solution of $\mathbf{1 0}(50.0 \mathrm{mg}, 0.156 \mathrm{mmol})$ in THF $(3.0 \mathrm{~mL})$ was added potassium thioacetate $(53.3 \mathrm{mg}, 0.468 \mathrm{mmol})$ and $\mathrm{BF}_{3} \square \mathrm{Et}_{2} \mathrm{O}(0.06 \mathrm{~mL}, 0.468 \mathrm{mmol})$ sequentially at $-78^{\circ} \mathrm{C}$ under a nitrogen atmosphere. The reaction mixture was warmed from $-78^{\circ} \mathrm{C}$ to $4^{\circ} \mathrm{C}$ by stirring for $24 \mathrm{~h}$ in a cooled environment $\left(4^{\circ} \mathrm{C}\right)$. The reaction was quenched by the addition of solid $\mathrm{NaHCO}_{3}$, and then the mixture was filtered. The filtrate was concentrated in vacuo and the residue was purified by column chromatography ( $n$-hexane/AcOEt=2:1) to give $9 \mathbf{a}(44.3 \mathrm{mg}, 78 \%)$ as an orange solid.

9a: mp 149-152 ${ }^{\circ} \mathrm{C}$ (from AcOEt). IR (KBr): v 1660, 1605, 1575, $1505 \mathrm{~cm}^{-1} \cdot{ }^{1} \mathrm{H}$ NMR (300 MHz): $\delta 2.03(\mathrm{dd}, 1 \mathrm{H}, J=14.0,5.0 \mathrm{~Hz}), 2.42$ (s, 3H), $2.43(\mathrm{dd}, 1 \mathrm{H}, J=14.0$, $5.0 \mathrm{~Hz}), 5.59-5.63(\mathrm{~m}, 1 \mathrm{H}), 6.38(\mathrm{~d}, 1 \mathrm{H}, J=9.9 \mathrm{~Hz}), 6.40(\mathrm{~d}, 1 \mathrm{H}, J=9.9 \mathrm{~Hz}), 6.86(\mathrm{br} \mathrm{s}$, $1 \mathrm{H}), 6.89(\mathrm{dd}, 1 \mathrm{H}, J=10.8,3.0 \mathrm{~Hz}), 7.09(\mathrm{dd}, 1 \mathrm{H}, J=10.8,3.0 \mathrm{~Hz}), 7.63(\mathrm{t}, 1 \mathrm{H}, J=7.0$ $\mathrm{Hz}), 7.70(\mathrm{t}, 1 \mathrm{H}, J=7.0 \mathrm{~Hz}), 7.99(\mathrm{~d}, 1 \mathrm{H}, J=9.0 \mathrm{~Hz}), 8.02(\mathrm{~d}, 1 \mathrm{H}, J=8.0 \mathrm{~Hz}) .{ }^{13} \mathrm{C}$ NMR (75 MHz): $\delta 30.9,38.4,39.7,52.6,110.8,126.2,126.5,128.2,128.5,129.8$, 132.4, 133.0, 135.2, 142.8, 151.6, 152.8, 179.9, 180.0, 185.2, 195.2. HRMS calcd for $\mathrm{C}_{20} \mathrm{H}_{13} \mathrm{NO}_{4} \mathrm{~S}\left(\mathrm{M}^{+}\right)$365.0722, found 365.0711.

\section{1,2,3,4,5,10-Hexahydrobenzo[6,7-g]quinoline-5,10-dione-2-sulfanyl-3'-4-spiro-}

\section{4'-cyclohexa-5'-en-1'-one (4).}

To a stirred solution of $9 \mathbf{a}(44.3 \mathrm{mg}, 0.121 \mathrm{mmol})$ in EtOH $(10 \mathrm{~mL})$ was added $2 \mathrm{M}$ $\mathrm{NH}_{3} / \mathrm{EtOH}$ solution $(0.61 \mathrm{~mL}, 1.21 \mathrm{mmol})$ at room temperature. The reaction mixture was stirred for $12 \mathrm{~h}$, then concentrated in vacuo. The residue was purified by column chromatography ( $n$-hexane/AcOEt=2:1) to give $4(20.0 \mathrm{mg}, 51 \%)$ as an orange solid. 
4: $\mathrm{mp} 255-257^{\circ} \mathrm{C}$ (from AcOEt). IR (KBr): y 1680, 1675, 1595, 1565, $1495 \mathrm{~cm}^{-1} .{ }^{1} \mathrm{H}$ NMR (300 MHz): $\delta 2.61-2.83(\mathrm{~m}, 4 \mathrm{H}), 4.60(\mathrm{dd}, J=12.0,7.5 \mathrm{~Hz}, 1 \mathrm{H}), 5.29-5.33(\mathrm{~m}$, 1H), 6.19 (d, $J=10.5 \mathrm{~Hz}, 1 \mathrm{H}), 6.49$ (br s, 1H), 7.05 (d, $J=10.5 \mathrm{~Hz}, 1 \mathrm{H}), 7.64$ (t, $J=8.0$ $\mathrm{Hz}, 1 \mathrm{H}), 7.75(\mathrm{t}, J=8.0 \mathrm{~Hz}, 1 \mathrm{H}), 8.02(\mathrm{~d}, J=8.0 \mathrm{~Hz}, 1 \mathrm{H}), 8.07(\mathrm{~d}, J=8.0 \mathrm{~Hz}, 1 \mathrm{H}) .{ }^{13} \mathrm{C}$ NMR (75 MHz, [D 6 ]DMSO): $\oint$ 39.4, 45.3, 46.8, 55.7, 59.5, 118.8, 124.9, 125.4, 125.7, $129.8,132.4,133.0,135.0,144.9,154.9,178.4,180.6,195.7$. UV/Vis $(\mathrm{MeOH})$ : $\lambda \max (\varepsilon)=473$ (1300), 270 (10900), $245 \mathrm{~nm}$ (10000). HRMS calcd for $\mathrm{C}_{18} \mathrm{H}_{13} \mathrm{NO}_{3} \mathrm{~S}$ $\left(\mathrm{M}^{+}\right)$323.0616, found 323.0621.

\section{1,2,3,4,5,10-Hexahydrobenzo[6,7-g]quinoline-5,10-dione-2-(4-methoxybenzyl-}

\section{sulfanyl)-4-spiro-4'-cyclohexa-2',5'-dien-1'-one (9b).}

To a stirred solution of $\mathbf{1 0}(15.7 \mathrm{mg}, 0.0489 \mathrm{mmol})$ in THF $(1.5 \mathrm{~mL})$ was added $p$ methoxybenzylthiol (MeOBnSH) $(6.8 \mu \mathrm{L}, 0.0489 \mathrm{mmol})$ and $\mathrm{BF}_{3} \square \mathrm{Et}_{2} \mathrm{O}(6.2 \mu \mathrm{L}, 0.0489$ mmol) sequentially at $-78^{\circ} \mathrm{C}$ under a nitrogen atmosphere. The reaction mixture was warmed from $-78^{\circ} \mathrm{C}$ to $4^{\circ} \mathrm{C}$ by stirring for $24 \mathrm{~h}$ in a cooled environment $\left(4^{\circ} \mathrm{C}\right)$. The reaction was quenched by the addition of solid $\mathrm{NaHCO}_{3}$, and the mixture was filtered. The filtrate was concentrated in vacuo and the residue was purified by column chromatography ( $n$-hexane/AcOEt=2:1) to give $\mathbf{9 b}(17.2 \mathrm{mg}, 80 \%)$ as a red solid.

9b: mp 180-182 ${ }^{\circ} \mathrm{C}$ (from AcOEt). IR (KBr): v 3348, 1659, 1605, 1574, 1514, 1504 $\mathrm{cm}^{-1} .{ }^{1} \mathrm{H}$ NMR $(300 \mathrm{MHz}): \oint 2.04(\mathrm{~d}, 2 \mathrm{H}, J=6.5 \mathrm{~Hz}), 3.80$ (s, 3H), 3.87 (s, 2H), 4.60 (td, 1H, $J=6.5,2.0), 6.16$ (br s, 1H), 6.32 (dd, 1H, $J=10.0,2.0 \mathrm{~Hz}), 6.37$ (dd, 1H, $J=$ 10.0, $2.0 \mathrm{~Hz}), 6.76(\mathrm{dd}, 1 \mathrm{H}, J=10.0,3.0 \mathrm{~Hz}), 6.88$ (d, 2H, $J=8.5 \mathrm{~Hz}), 7.09$ (dd, 1H, $J$ $=10.0,3.0 \mathrm{~Hz}), 7.25(\mathrm{~d}, 2 \mathrm{H}, J=8.5 \mathrm{~Hz}), 7.58(\mathrm{td}, 1 \mathrm{H}, J=8.0,1.5 \mathrm{~Hz}), 7.68(\mathrm{td}, 1 \mathrm{H}, J=$ 7.5, $1.5 \mathrm{~Hz}), 7.97(\mathrm{dd}, 1 \mathrm{H}, J=7.5,1.5 \mathrm{~Hz}), 7.99(\mathrm{dd}, 1 \mathrm{H}, J=7.5,1.5 \mathrm{~Hz}),{ }^{13} \mathrm{C} \mathrm{NMR}(75$ $\mathrm{MHz}): \oint 30.9,38.4,39.7,52.6,110.8,126.2,126.5,128.2,128.5,129.8,132.4,133.0$, 135.2, 142.8, 151.6, 152.8, 179.9, 180.0, 185.2, 195.2. HRMS calcd for $\mathrm{C}_{26} \mathrm{H}_{21} \mathrm{NO}_{4} \mathrm{~S}$ $\left(\mathrm{M}^{+}\right)$443.1191, found 443.1173. 


\section{1,2,3,4,5,10-Hexahydrobenzo[6,7-g]quinoline-5,10-dione-2-sulfanyl-3'-4-spiro- 4'-cyclohexa-5'-en-1'-one (4).}

To a stirred solution of $9 \mathbf{b}(25.6 \mathrm{mg}, 0.0577 \mathrm{mmol})$ in $\mathrm{CH}_{2} \mathrm{Cl}_{2}(2.0 \mathrm{~mL})$ was added $30 \% \mathrm{HBr}-\mathrm{AcOH}(0.05 \mathrm{~mL})$ at $-78^{\circ} \mathrm{C}$ under a nitrogen atmosphere. The reaction mixture was warmed from $-78^{\circ} \mathrm{C}$ to $4^{\circ} \mathrm{C}$ by stirring for $3.5 \mathrm{~h}$ in a cooled environment $\left(4^{\circ} \mathrm{C}\right)$. The reaction was quenched by the addition of solid $\mathrm{NaHCO}_{3}$, and then the mixture was filtered. The filtrate was concentrated in vacuo and the residue was purified by preparative TLC ( $n$-hexane/AcOEt=1:1) to give $4(7.7 \mathrm{mg}, 41 \%)$ as a red solid.

\section{3-(3-Bromo-4-hydroxyphenyl)-2-(tritylamino)propionic acid methyl ester (15).}

To a solution of $\mathbf{6} \square \mathbf{H C l}(511 \mathrm{mg}, 2.21 \mathrm{mmol})$ in DMF $(20 \mathrm{~mL})$ was added triethylamine $(0.62 \mathrm{~mL}, 4.44 \mathrm{mmol})$ and tritylchloride $(617 \mathrm{mg}, 2.21 \mathrm{mmol})$ sequentially at $0^{\circ} \mathrm{C}$. The reaction mixture was stirred at room temperature for $3 \mathrm{~h}$. The mixture was then quenched with water and diluted with AcOEt. The layers were separated, and the aqueous layer was extracted twice with AcOEt. The combined organics were washed with brine, dried, and concentrated in vacuo. The residue was purified by flash chromatography ( $n$-hexane/AcOEt $=3 / 1)$ to give tritylated tyrosine methyl ester (1.003 g, quant.) as a colorless amorphous.

IR (KBr): v 3345, 1715, 1615, 1595, 1515, $1445 \mathrm{~cm}^{-1} .{ }^{1} \mathrm{H}$ NMR (300 MHz): $\delta 2.59$ (d, $1 \mathrm{H}, J=10.8 \mathrm{~Hz}), 2.88(\mathrm{dd}, 2 \mathrm{H}, J=6.9,4.2 \mathrm{~Hz}), 3.04(\mathrm{~s}, 3 \mathrm{H}), 3.50(\mathrm{dt}, 1 \mathrm{H}, J=10.8,6.9$ $\mathrm{Hz}), 4.78(\mathrm{~s}, 1 \mathrm{H}), 6.76(\mathrm{~d}, 2 \mathrm{H}, J=8.4 \mathrm{~Hz}), 7.06(\mathrm{~d}, 2 \mathrm{H}, J=8.4 \mathrm{~Hz}), 7.11-7.26(\mathrm{~m}, 9 \mathrm{H})$, $7.40(\mathrm{~d}, 6 \mathrm{H}, J=7.5 \mathrm{~Hz}) .{ }^{13} \mathrm{C} \mathrm{NMR}(75 \mathrm{MHz}): \delta 41.4,51.4,58.4,70.9,115.1,126.3$, $127.7,128.7,129.2,130.8,145.8,154.5,175.3$. HR-FABMS calcd for $\mathrm{C}_{29} \mathrm{H}_{28} \mathrm{NO}_{3}$ $\left(\mathrm{M}+\mathrm{H}^{+}\right)$; 438.2069, found 438.2073. Anal. calcd. for $\mathrm{C}_{29} \mathrm{H}_{27} \mathrm{NO}_{3} \bullet 0.4 \mathrm{H}_{2} \mathrm{O}: \mathrm{C} 79.61, \mathrm{H}$ 6.22, N 3.20; found C 78.14, H 6.52, N 3.02.

To a stirred solution of tritylated tyrosine methyl ester (3.28 g, $7.5 \mathrm{mmol})$ in DMF (40 mL) was added dropwise slowly a solution of NBS (1.34 g, $7.5 \mathrm{mmol})$ in DMF at room temperature under a nitrogen atmosphere. The reaction mixture was stirred for 2.5 h. The mixture was then quenched with water and diluted with $\mathrm{Et}_{2} \mathrm{O}$. The layers were separated, and the aqueous layer was extracted twice with $\mathrm{Et}_{2} \mathrm{O}$. The combined organics 
were washed twice with water and once with brine, dried, and concentrated in vacuo. The residue was purified by flash chromatography ( $n$-hexane/AcOEt=4/1) to give $\mathbf{1 5}$ $(2.546 \mathrm{~g}, 65 \%)$ as a colorless amorphous.

15: IR (KBr): v 3500, 3340, 1730, 1605, 1595, 1495, $1445 \mathrm{~cm}^{-1} .{ }^{1} \mathrm{H}$ NMR (300 MHz): $\delta 2.57(\mathrm{~d}, 1 \mathrm{H}, J=10.8 \mathrm{~Hz}), 2.85(\mathrm{~d}, 2 \mathrm{H}, J=6.6 \mathrm{~Hz}), 3.06(\mathrm{~s}, 3 \mathrm{H}), 3.49-3.52(\mathrm{~m}, 1 \mathrm{H})$, $5.43(\mathrm{~s}, 1 \mathrm{H}), 6.95(\mathrm{~d}, 1 \mathrm{H}, J=8.4 \mathrm{~Hz}), 7.04(\mathrm{dd}, 1 \mathrm{H}, J=8.4,1.8 \mathrm{~Hz}), 7.12-7.31(\mathrm{~m}, 9 \mathrm{H})$, $7.32(\mathrm{~d}, 1 \mathrm{H}, J=1.8 \mathrm{~Hz}), 7.39(\mathrm{~d}, 6 \mathrm{H}, J=6.9 \mathrm{~Hz}) .{ }^{13} \mathrm{C} \mathrm{NMR}(75 \mathrm{MHz}): \delta 40.9,51.4$, 58.0, 70.9, 109.7, 115.7, 126.4, 127.8, 128.7, 130.5, 131.0, 133.1, 145.7, 151.1, 174.7. HR-FABMS calcd for $\mathrm{C}_{29} \mathrm{H}_{27} \mathrm{BrNO}_{3}\left(\mathrm{M}+\mathrm{H}^{+}\right) ; 516.1174$, found 516.1166. Anal. calcd. for $\mathrm{C}_{29} \mathrm{H}_{26} \mathrm{BrNO}_{3} \bullet 0.2 \mathrm{H}_{2} \mathrm{O}$ : C 67.15, H 5.09, $\mathrm{N} 2.70, \mathrm{Br} 15.40$; found $\mathrm{C} 66.90, \mathrm{H} 5.21, \mathrm{~N}$ 2.46, $\mathrm{Br} 15.08$.

\section{3-(3-Bromo-4-hydroxy-phenyl)-2-(8-oxo-1,3,4,8-tetrahydropyrrolo[4,3,2-de]} quinolin-7-ylamino) propionic acid methyl ester (17).

$0.1 \mathrm{M} \mathrm{MeOH}$ solution of $\mathrm{HCl}(0.9 \mathrm{~mL})$ was added to $15(18.1 \mathrm{mg}, 0.0350 \mathrm{mmol})$ at room temperature under a nitrogen atmosphere and the mixture was stirred for $30 \mathrm{~min}$. The above mixture was added dropwise to a stirred solution of 7-methoxy-3,4-dihydro1H-pyrrolo[4,3,2-de]quinolin-8-one (16a) $(7.0 \mathrm{mg}, 0.0346 \mathrm{mmol})$ in $\mathrm{MeOH}(0.5 \mathrm{~mL})$ at room temperature under a nitrogen atmosphere. The mixture was stirred for $18 \mathrm{~h}$ under the same conditions and was then concentrated in vacuo. The residue was purified by flash chromatography $\left(\mathrm{CH}_{2} \mathrm{Cl}_{2} / \mathrm{MeOH} / \mathrm{CF}_{3} \mathrm{CO}_{2} \mathrm{H}=100: 5: 0.1\right)$ to give 17•TFA (7.3 $\mathrm{mg}$, $37 \%$ ) as a red amorphous.

17•TFA: ${ }^{1} \mathrm{H}$ NMR $\left(270 \mathrm{MHz}, \mathrm{CD}_{3} \mathrm{OD}\right): \delta 2.95$ (t, $\left.2 \mathrm{H}, J=7.5 \mathrm{~Hz}\right), 3.08(\mathrm{dd}, 1 \mathrm{H}, J=$ 14.0, 8.0 Hz ), $3.26(\mathrm{dd}, 1 \mathrm{H}, J=14.0,5.0 \mathrm{~Hz}), 3.77$ (s, 3H), $3.86(\mathrm{t}, 2 \mathrm{H}, J=7.5 \mathrm{~Hz})$, $4.56(\mathrm{dd}, 1 \mathrm{H}, J=8.0,5.5 \mathrm{~Hz}), 5.35$ (br s, $1 \mathrm{H}), 6.79(\mathrm{~d}, 1 \mathrm{H}, J=8.5 \mathrm{~Hz}), 7.00(\mathrm{dd}, 1 \mathrm{H}, J$ $=8.5,2.0 \mathrm{~Hz}), 7.14(\mathrm{~s}, 1 \mathrm{H}), 7.30(\mathrm{~d}, 1 \mathrm{H}, J=2.0 \mathrm{~Hz})$. HR-FABMS calcd for $\mathrm{C}_{20} \mathrm{H}_{19} \mathrm{~N}_{3} \mathrm{O} 4 \mathrm{Br}\left(\mathrm{M}+\mathrm{H}^{+}\right)$; 444.0559, found 444.0532.

[2-[3-Bromo-4-(tert-butyldimethylsilyloxy)phenyl]-1-(tert-butyldimethylsilyloxymethyl)ethyl]tritylamine (19a). 
To a stirred solution of $\mathbf{1 5}$ (1.3749 g, $2.66 \mathrm{mmol})$ in $\mathrm{CH}_{2} \mathrm{Cl}_{2}(50 \mathrm{~mL})$ was added dropwise slowly a solution of diisobutylaluminum hydride (DIBAH) 1.0 M solution in hexane $(8.6 \mathrm{~mL}, 8.6 \mathrm{mmol})$ at $-78^{\circ} \mathrm{C}$ under a nitrogen atmosphere. The reaction mixture was stirred for $5 \mathrm{~h}$ at $-78^{\circ} \mathrm{C}$ to room temperature. The mixture was then quenched with water at $0^{\circ} \mathrm{C}$ and filtered through celite. The filtrate was washed with aqueous saturated $\mathrm{NaHCO}_{3}$ and then with brine, dried, and concentrated in vacuo. The residue was purified by flash chromatography $(n$-hexane/AcOEt $=2 / 1)$ to give monobrominated $N$ tritylated $(L)$-tyrosinol (1.246 g, 96\%) as a colorless amorphous.

IR (KBr): v 3505, 3330, 1595, 1495, $1445 \mathrm{~cm}^{-1} .{ }^{1} \mathrm{H}$ NMR (300 MHz): $\delta 1.96$ (br s, 1H), $2.15(\mathrm{dd}, 1 \mathrm{H}, J=13.2,4.8 \mathrm{~Hz}), 2.42(\mathrm{dd}, 1 \mathrm{H}, J=13.2,9.6 \mathrm{~Hz}), 2.70-2.74(\mathrm{~m}, 1 \mathrm{H})$, $2.97(\mathrm{dd}, 1 \mathrm{H}, J=10.8,4.2 \mathrm{~Hz}), 3.10(\mathrm{dd}, 1 \mathrm{H}, J=10.8,2.7 \mathrm{~Hz}), 6.76(\mathrm{dd}, 1 \mathrm{H}, J=8.4$, $1.8 \mathrm{~Hz}), 6.81(\mathrm{~d}, 1 \mathrm{H}, J=8.4 \mathrm{~Hz}), 6.98(\mathrm{~d}, 1 \mathrm{H}, J=1.8 \mathrm{~Hz}), 7.17-7.31(\mathrm{~m}, 9 \mathrm{H}), 7.54(\mathrm{~d}$, $6 \mathrm{H}, J=7.5 \mathrm{~Hz}) .{ }^{13} \mathrm{C}$ NMR $(75 \mathrm{MHz}): \delta 37.8,55.2,62.3,71.3,109.9,115.7,126.6$, 127.9, 128.6, 130.1, 132.6, 132.7, 146.4, 150.6. HR-FABMS calcd for $\mathrm{C}_{28} \mathrm{H}_{27} \mathrm{BrNO}_{2}$ $\left(M+\mathrm{H}^{+}\right) ; 488.1225$, found 488.1257 .

To a stirred solution of monobrominated $N$-tritylated $(L)$-tyrosinol (1.246 g, 2.55 $\mathrm{mmol})$ in $\mathrm{CH}_{2} \mathrm{Cl}_{2}(20 \mathrm{~mL})$ was added $\mathrm{DBU}(1.90 \mathrm{~mL}, 12.7 \mathrm{mmol})$ and $\mathrm{TBSCl}(1.166 \mathrm{~g}$, $7.74 \mathrm{mmol}$ ) sequentially at $0^{\circ} \mathrm{C}$ under a nitrogen atmosphere. The reaction mixture was stirred for $2.5 \mathrm{~h}$ at $0^{\circ} \mathrm{C}$. The mixture was then quenched with aqueous saturated $\mathrm{NaHCO}_{3}$ and extracted with $\mathrm{CH}_{2} \mathrm{Cl}_{2}$. The combined organics were washed with brine, dried, and concentrated in vacuo. The residue was purified by flash chromatography ( $n$ hexane/AcOEt=20/1) to give 19a $(1.583 \mathrm{~g}, 87 \%)$ as a colorless oil.

19a: IR (KBr): v 3320, 1600, 1495, 1470, $1460 \mathrm{~cm}^{-1} .{ }^{1} \mathrm{H}$ NMR (300 MHz): $\delta-0.13$ (s, $3 \mathrm{H}),-0.11(\mathrm{~s}, 3 \mathrm{H}), 0.20(\mathrm{~s}, 6 \mathrm{H}), 0.85(\mathrm{~s}, 9 \mathrm{H}), 1.02(\mathrm{~s}, 9 \mathrm{H}), 2.14(\mathrm{~d}, 1 \mathrm{H}, J=6.3 \mathrm{~Hz})$, 2.46-2.61 (m, 3H), 2.68 (br s, 1H), 2.90 (dd, 1H, $J=9.6,3.0 \mathrm{~Hz}), 6.69$ (d, 1H, $J=8.4$ $\mathrm{Hz}), 6.80(\mathrm{dd}, 1 \mathrm{H}, J=8.4,1.8 \mathrm{~Hz}), 7.18(\mathrm{~d}, 1 \mathrm{H}, J=1.8 \mathrm{~Hz}), 7.15-7.29(\mathrm{~m}, 9 \mathrm{H}), 7.56(\mathrm{~d}$, $6 \mathrm{H}, J=7.5 \mathrm{~Hz}) .{ }^{13} \mathrm{C}$ NMR $(75 \mathrm{MHz}): \delta-5.5,-5.4,-4.3,18.1,18.3,25.7,25.9,37.7$, $55.2,62.3,71.1,114.8,119.7,126.3,127.8,128.7,129.3,133.9,134.3,147.2,150.6$. 
HR-FABMS calcd for $\mathrm{C}_{40} \mathrm{H}_{33} \mathrm{BrNO}_{2} \mathrm{Si}_{2}\left(\mathrm{M}+\mathrm{H}^{+}\right)$; 716.2955, found 716.2942. Anal. calcd. for $\mathrm{C}_{40} \mathrm{H}_{34} \mathrm{BrNO}_{2} \mathrm{Si}_{2}$ : C 67.01, H 7.59, N 1.95, Br 11.15; found C 67.02, H 7.58, N 1.77, Br 10.89 .

\section{[2-[3-bromo-4-hydroxyphenyl]-1-(tert-butyldimethylsilyloxymethyl)ethyl] tritylamine (19b).}

To a stirred solution of 19a $(1.577 \mathrm{~g}, 2.20 \mathrm{mmol})$ in THF $(35 \mathrm{~mL})$ was added TBAF $\left(2.2 \mathrm{~mL}(1.0 \mathrm{M}\right.$ solution in THF), $2.20 \mathrm{mmol})$ at $0^{\circ} \mathrm{C}$ under a nitrogen atmosphere. The reaction mixture was stirred for $0.5 \mathrm{~h}$ at $0^{\circ} \mathrm{C}$. The mixture was then quenched with aqueous saturated $\mathrm{NH}_{4} \mathrm{Cl}$ and extracted with AcOEt. The combined organics were washed with brine, dried, and concentrated in vacuo. The residue was purified by flash chromatography ( $n$-hexane/AcOEt=10/1) to give $\mathbf{1 9 b}(1.330 \mathrm{~g}$, quant.) as a colorless oil.

19b: IR (KBr): v 3520, 3325, 1595, 1495, 1470, $1450 \mathrm{~cm}^{-1} .{ }^{1} \mathrm{H}$ NMR (300 MHz): $\delta-$ $0.13(\mathrm{~s}, 3 \mathrm{H}),-0.11$ (s, 3H), 0.85 (s, 9H), 2.10 (br s, 1H), 2.47-2.61 (m, 3H), 2.69 (br s, $1 \mathrm{H}), 2.88(\mathrm{dd}, 1 \mathrm{H}, J=9.6,3.0 \mathrm{~Hz}), 5.40(\mathrm{br} \mathrm{s}, 1 \mathrm{H}), 6.85-6.86(\mathrm{~m}, 2 \mathrm{H}), 7.13$ (d, 1H, $J=$ $1.8 \mathrm{~Hz}), 7.12-7.29(\mathrm{~m}, 9 \mathrm{H}), 7.56(\mathrm{~d}, 6 \mathrm{H}, J=7.5 \mathrm{~Hz}) .{ }^{13} \mathrm{C} \mathrm{NMR}(75 \mathrm{MHz}): \delta-5.5,-5.4$, $18.1,25.9,37.6,55.1,62.3,71.1,109.7,115.5,126.3,127.8,128.7,130.3,132.9,133.3$, 147.1, 150.3. HR-FABMS calcd for $\mathrm{C}_{34} \mathrm{H}_{41} \mathrm{BrNO}_{2} \mathrm{Si}\left(M+\mathrm{H}^{+}\right) ; 602.2090$, found 602.2075. Anal. calcd. for $\mathrm{C}_{34} \mathrm{H}_{41} \mathrm{BrNO}_{2} \mathrm{Si}$ : C 67.76, H 6.69, N 2.32, Br 13.26; found C 68.11, H 6.79, N 2.29, Br 12.64.

7-Methoxy-1-(toluene-4-sulfonyl)-3,4-dihydro-1H-pyrrolo[4,3,2-de]quinolin-8one (16b).

To a stirred solution of 3-(2-azidoethyl)-6,7-dimethoxy-1-[(4-methylphenyl)sulfonyl] indole ${ }^{6 \mathrm{~b}}(215.4 \mathrm{mg}, 0.538 \mathrm{mmol})$ in $\left(\mathrm{CF}_{3}\right)_{2} \mathrm{CHOH}(15 \mathrm{~mL})-\mathrm{H}_{2} \mathrm{O}(0.3 \mathrm{~mL})$ was added drpwise trimethylsilyl trifluoromethane sulfonate $(0.23 \mathrm{~mL}, 1.271 \mathrm{mmol})$ follwed by PIFA $(277.4 \mathrm{mg}, 0.645 \mathrm{mmol})$ at $0^{\circ} \mathrm{C}$ under a nitrogen atmosphere. After stirring for $1.5 \mathrm{~h}$ at $0{ }^{\circ} \mathrm{C}$, the mixture was quenched with saturated aqueous $\mathrm{NaHCO}_{3}$, stirred for $15 \mathrm{~min}$, and then extracted with $\mathrm{CH}_{2} \mathrm{Cl}_{2}$. The combined organics were washed with brine, 
dried, and concentrated in vacuo. The residue was purified by flash chromatography $\left(\mathrm{CH}_{2} \mathrm{Cl}_{2} / \mathrm{MeOH} / \mathrm{Et}_{3} \mathrm{~N}=100: 5: 0.1\right)$ to give $\mathbf{1 6 b}(144.9 \mathrm{mg}, 76 \%)$ as a yellow solid.

IR (KBr): v 1670, 1620, 1595, 1580, $1535 \mathrm{~cm}^{-1} .{ }^{1} \mathrm{H}$ NMR $\left(\mathrm{CDCl}_{3}\right): \delta 2.40(\mathrm{~s}, 3 \mathrm{H})$, $2.78(\mathrm{t}, 2 \mathrm{H}, J=7.6 \mathrm{~Hz}), 3.78(\mathrm{~s}, 3 \mathrm{H}), 4.18(\mathrm{t}, 2 \mathrm{H}, J=7.6 \mathrm{~Hz}), 6.09(\mathrm{~s}, 1 \mathrm{H}), 7.31(\mathrm{~d}, 2 \mathrm{H}$, $J=8.2 \mathrm{~Hz}), 7.51(\mathrm{~s}, 1 \mathrm{H}), 8.08(\mathrm{~d}, 2 \mathrm{H}, J=8.2 \mathrm{~Hz})$. HRMS calcd for $\mathrm{C}_{18} \mathrm{H}_{16} \mathrm{~N}_{2} \mathrm{O}_{4} \mathrm{~S}\left(\mathrm{M}^{+}\right)$ 356.0831, found 356.0825. This compound gradually decomposed during ${ }^{13} \mathrm{C} \mathrm{NMR}$ measurement due to its unstability.

\section{7-[2-(3-Bromo-4-hydroxyphenyl)-1-(tert-butyldimethylsilyloxymethyl)-} ethylamino]-1-(toluene-4-sulfonyl)-3,4-dihydro-1H-pyrrolo[4,3,2-de]quinonlin-8one (20a).

$0.1 \mathrm{~N} \mathrm{MeOH}$ solution of $\mathrm{HCl}(5.2 \mathrm{~mL})$ was added to $19 \mathrm{~b}(312.4 \mathrm{mg}, 0.518 \mathrm{mmol})$ at room temperature under a nitrogen atmosphere and the mixture was stirred for $30 \mathrm{~min}$. The above mixture was added dropwise to a stirred solution of $\mathbf{1 6 b}$ (144.9 $\mathrm{mg}, 0.407$ $\mathrm{mmol})$ in $\mathrm{MeOH}(1.0 \mathrm{~mL})$ at room temperature under a nitrogen atmosphere. The mixture was stirred for $16 \mathrm{~h}$ under the same conditions and was then concentrated in vacuo. The residue was purified by flash chromatography $\left(\mathrm{CH}_{2} \mathrm{Cl}_{2} / \mathrm{MeOH} / \mathrm{Et}_{3} \mathrm{~N}=100: 5: 0.1\right)$ to give $\mathbf{2 0 a}(149.8 \mathrm{mg}, 54 \%)$ as a red amorphous solid.

20a: IR (KBr): v 3370, 1665, 1610, 1580, 1550, 1535, 1495, 1460, 1445, $1380 \mathrm{~cm}^{-1}$. ${ }^{1} \mathrm{H}$ NMR (500 MHz, CD $\left.3 \mathrm{OD}\right): \delta 0.01(\mathrm{~s}, 6 \mathrm{H}), 0.89(\mathrm{~s}, 9 \mathrm{H}), 2.41(\mathrm{~s}, 3 \mathrm{H}), 2.71-2.81(\mathrm{~m}$, 4H), 3.58-3.61 (m, 2H), 3.66 (dd, 1H, $J=10.5,4.5 \mathrm{~Hz}$ ), 3.97 (br s, 2H), 5.49 (br s, 1H), $6.76(\mathrm{~d}, 1 \mathrm{H}, J=8.5 \mathrm{~Hz}), 6.99(\mathrm{dd}, 1 \mathrm{H}, J=8.5,2.0 \mathrm{~Hz}), 7.28(\mathrm{~d}, 1 \mathrm{H}, J=2.0 \mathrm{~Hz}), 7.39(\mathrm{~d}$, $2 \mathrm{H}, J=8.5 \mathrm{~Hz}$ ), 7.66 (br s, $1 \mathrm{H}), 8.00$ (d, 2H, $J=8.5 \mathrm{~Hz}) .{ }^{13} \mathrm{C} \mathrm{NMR}\left(125 \mathrm{MHz}, \mathrm{CD}_{3} \mathrm{OD}\right.$, as its $\mathrm{HCl}$ salt): $\delta-5.74,19.0,21.8,26.3,36.5,43.5,49.9,59.9,63.5,86.8,110.7,117.4$, 119.9, 124.2, 129.0, 130.1, 130.2, 130.7, 131.1, 131.2, 134.7, 134.9, 148.6, 154.2, 157.8, 166.9. HR-FABMS calcd for $\mathrm{C}_{32} \mathrm{H}_{39} \mathrm{BrN}_{3} \mathrm{O}_{3} \mathrm{SSi}\left(M+\mathrm{H}^{+}\right)$; 684.1563, found 684.1586. 


\section{5-(Toluene-4-sulfonyl)-3,5,7,8,9,10-hexahydro-2H-1,5,7-triaza-}

acephenanthrylen-6-one-8-(tert-butyldimethylsilyloxymethyl)-10-spiro-4'-2'bromocyclohexa-2',5'-dien-1'-one (21).

To a stirred solution of 20a $(110.7 \mathrm{mg}, 0.162 \mathrm{mmol})$ in $\mathrm{CF}_{3} \mathrm{CH}_{2} \mathrm{OH}(10 \mathrm{~mL})$ was added MK10 (50 mg) and PIFA (90.4 $\mathrm{mg}, 0.210 \mathrm{mmol})$ sequentially at room temperature. The mixture was stirred for $30 \mathrm{~min}$. under the same conditions and then concentrated in vacuo. The residue was purified by flash chromatography ( $n$ hexane/AcOEt $\left./ \mathrm{Et}_{3} \mathrm{~N}=100: 50: 0.1\right)$ to afford two diastereomers $21 \mathrm{a}(30 \mathrm{mg}, 27 \%)$ and $\mathbf{2 1 ' a}(20 \mathrm{mg}, 18 \%$ ) respectively as a red amorphous solid.

21a (less polar): IR (KBr): v 3390, 1660, 1595, 1575, 1525, 1460, 1435, $1380 \mathrm{~cm}^{-1}$. ${ }^{1} \mathrm{H}$ NMR (500 MHz): $\delta 0.08$ (s, 3H), $0.10(\mathrm{~s}, 3 \mathrm{H}), 0.92(\mathrm{~s}, 9 \mathrm{H}), 1.57$ (d, 1H, J = 13.5 $\mathrm{Hz}), 1.72(\mathrm{t}, 1 \mathrm{H}, J=11.5 \mathrm{~Hz}), 2.40$ (s, 3H), 2.60 (t, 2H, $J=8.0 \mathrm{~Hz}), 3.49-3.52$ (m, 2H), 3.71-3.72 (m, 1H), $3.83(\mathrm{dt}, 1 \mathrm{H}, J=8.5,17.5 \mathrm{~Hz}), 4.09(\mathrm{dt}, 1 \mathrm{H}, J=7.0,19.5 \mathrm{~Hz}), 6.07$ (br s, 1H), 6.27 (d, 1H, $J=9.5 \mathrm{~Hz}), 6.96(\mathrm{dd}, 1 \mathrm{H}, J=9.5,2.5 \mathrm{~Hz}), 7.20$ (d, 1H, $J=3.0$ $\mathrm{Hz}), 7.30(\mathrm{~d}, 2 \mathrm{H}, J=8.5 \mathrm{~Hz}), 7.43(\mathrm{~s}, 1 \mathrm{H}), 8.02(\mathrm{~d}, 2 \mathrm{H}, J=8.5 \mathrm{~Hz}) .{ }^{13} \mathrm{C}$ NMR $(125$ MHz): $\delta$-5.4, 17.5, 18.3, 21.7, 25.9, 36.6, 43.9, 49.6, 66.1, 104.2, 118.5, 121.9, 125.5, $125.9,126.1,128.7,129.7,134.7,142.0,145.7,152.2,152.7,157.1,168.4,179.0$. UV/Vis $\left(\mathrm{CH}_{2} \mathrm{Cl}_{2}\right): \lambda \max (\varepsilon)=487(2000), 330(11300), 234 \mathrm{~nm}$ (27800). HR-FABMS calcd for $\mathrm{C}_{32} \mathrm{H}_{37} \mathrm{BrN}_{3} \mathrm{O}_{3} \mathrm{SSi}\left(M+\mathrm{H}^{+}\right) ; 682.1407$, found 682.1392 . $[\alpha]_{D}^{23.0}+149.8^{\circ}$ (c $\left.0.46, \mathrm{CHCl}_{3}\right)$.

21'a (polar): IR (KBr): v 3390, 1660, 1595, 1575, 1525, 1460, 1435, $1380 \mathrm{~cm}^{-1} .{ }^{1} \mathrm{H}$ NMR (300 MHz): $\delta 0.10(\mathrm{~s}, 3 \mathrm{H}), 0.12(\mathrm{~s}, 3 \mathrm{H}), 0.95(\mathrm{~s}, 9 \mathrm{H}), 1.60(\mathrm{~m}, 1 \mathrm{H}), 1.73(\mathrm{t}, 1 \mathrm{H}, J$ $=12.0 \mathrm{~Hz}), 2.43(\mathrm{~s}, 3 \mathrm{H}), 2.61(\mathrm{t}, 2 \mathrm{H}, J=7.0 \mathrm{~Hz}), 3.48-3.60(\mathrm{~m}, 2 \mathrm{H}), 3.74-3.86(\mathrm{~m}, 2 \mathrm{H})$, 4.07 (dt, 1H, $J=17.7,6.5 \mathrm{~Hz}), 6.12(\mathrm{br} \mathrm{s}, 1 \mathrm{H}), 6.33(\mathrm{~d}, 1 \mathrm{H}, J=9.9 \mathrm{~Hz}), 6.91$ (dd, 1H, $J$ =9.9, $2.7 \mathrm{~Hz}), 7.32(\mathrm{~d}, 2 \mathrm{H}, J=8.1 \mathrm{~Hz}), 7.42(\mathrm{~d}, 1 \mathrm{H}, J=2.4 \mathrm{~Hz}), 7.45(\mathrm{~s}, 1 \mathrm{H}), 8.03(\mathrm{~d}$, $2 \mathrm{H}, J=8.4 \mathrm{~Hz}) .{ }^{13} \mathrm{C}$ NMR $(125 \mathrm{MHz}): \delta-5.4,-5.3,17.6,18.4,21.7,25.9,36.8,44.0$, 49.6, 66.1, 103.9, 118.6, 121.9, 123.9, 125.0, 125.6, 126.1, 128.7, 129.7, 134.7, 142.2, $145.8,152.3,152.8,157.1,168.4,179.0$. UV/Vis $\left(\mathrm{CH}_{2} \mathrm{Cl}_{2}\right): \lambda \max (\varepsilon)=486(2200)$, 
331(12200), $233 \mathrm{~nm}$ (26900). HR-FABMS calcd for $\mathrm{C}_{32} \mathrm{H}_{37} \mathrm{BrN}_{3} \mathrm{O}_{3} \mathrm{SSi}\left(M+\mathrm{H}^{+}\right)$; 682.1407, found 682.1413. $[\alpha]_{\mathrm{D}}^{25.0}+107.7^{\circ}\left(\mathrm{c} 0.34, \mathrm{CHCl}_{3}\right)$.

\section{3,5,7,8,9,10-hexahydro-2H-1,5,7-triaza-acephenanthrylen-6-one-8-methylene[5']} ether-10-spiro-4'-(6'-bromo)-cyclohex-2'-en-1'-one (23a).

To a stirred solution of $21 \mathrm{a}$ (74.3 $\mathrm{mg}, 0.109 \mathrm{mmol})$ in $\mathrm{CH}_{2} \mathrm{Cl}_{2}(7 \mathrm{~mL})$ was added $\mathrm{BF}_{3} \square$ $\mathrm{Et}_{2} \mathrm{O}(0.28 \mathrm{~mL}, 2.18 \mathrm{mmol})$ at $0^{\circ} \mathrm{C}$ under a nitrogen atmosphere. The reaction mixture was stirred for $4 \mathrm{~h}$ at $0^{\circ} \mathrm{C}$ then to room temperature. The mixture was quenched with $\mathrm{NaHCO}_{3}$, filtered and then concentrated in vасио. The residue was purified by flash chromatography $\left(\mathrm{CH}_{2} \mathrm{Cl}_{2} / \mathrm{MeOH}=20 / 1\right)$ to give $22(54.5 \mathrm{mg}, 88 \%)$ as a red amorphous.

22: IR (KBr): v 3390, 1660, 1570, 1525, 1485, 1460, $1375 \mathrm{~cm}^{-1} .{ }^{1} \mathrm{H}$ NMR (300 MHz, $\left.\left(\mathrm{CD}_{3}\right)_{2} \mathrm{CO}\right): \delta 1.65(\mathrm{dd}, 1 \mathrm{H}, J=13.0,3.0 \mathrm{~Hz}), 1.91(\mathrm{t}, 1 \mathrm{H}, J=12.0 \mathrm{~Hz}), 2.43(\mathrm{~s}, 3 \mathrm{H})$, $2.65(\mathrm{dd}, 2 \mathrm{H}, J=8.0,7.0 \mathrm{~Hz}), 3.53-3.66(\mathrm{~m}, 2 \mathrm{H}), 3.74-3.86(\mathrm{~m}, 2 \mathrm{H}), 4.05(\mathrm{dt}, 1 \mathrm{H}, J=$ 18.0, 7.0 Hz), 6.17 (d, 1H, J=10.0 Hz), 6.25 (br s, 1H), 7.22 (dd, 1H, $J=10.0,2.5 \mathrm{~Hz})$, $7.46(\mathrm{~d}, 1 \mathrm{H}, J=3.0 \mathrm{~Hz}), 7.47$ (d, 2H, $J=7.5 \mathrm{~Hz}), 7.64$ (s, 1H), 8.06 (d, 2H, $J=8.0 \mathrm{~Hz})$. ${ }^{13} \mathrm{C}$ NMR $\left(75 \mathrm{MHz},\left(\mathrm{CD}_{3}\right)_{2} \mathrm{CO}\right): \delta 18.0,21.6,37.2,45.0,50.3,50.4,65.3,105.0,119.6$, $122.2,122.6,126.9,127.5,128.2,129.4,130.2,130.3,130.7,135.7,143.2,147.1,154.1$, 158.6, 169.3, 178.8. UV/Vis $\left(\mathrm{CH}_{2} \mathrm{Cl}_{2}\right): \lambda \max (\varepsilon)=486(1600), 330(10000), 230 \mathrm{~nm}$ (28000). HR-FABMS calcd for $\mathrm{C}_{26} \mathrm{H}_{23} \mathrm{BrN}_{3} \mathrm{O}_{3} \mathrm{~S}\left(M+\mathrm{H}^{+}\right)$; 568.0542, found 568.0539. $[\alpha]_{\mathrm{D}}^{24.9}+182^{\circ}\left(\mathrm{c} 0.133, \mathrm{CHCl}_{3}\right)$.

To a stirred solution of $22(24.2 \mathrm{mg}, 0.0426 \mathrm{mmol})$ in $\mathrm{CH}_{2} \mathrm{Cl}_{2}(2.7 \mathrm{~mL})$ was added $30 \% \mathrm{HBr}-\mathrm{AcOH}(0.04 \mathrm{~mL})$ at $-78^{\circ} \mathrm{C}$ under a nitrogen atmosphere. The reaction mixture was warmed from $-78^{\circ} \mathrm{C}$ to $4^{\circ} \mathrm{C}$ by stirring for $36 \mathrm{~h}$ in a cooled environment $\left(4^{\circ} \mathrm{C}\right)$. The mixture was quenched with aqueous saturated $\mathrm{NaHCO}_{3}$ and extracted with $\mathrm{CH}_{2} \mathrm{Cl}_{2}$. The aqueous layer was treated with saturated aqueous $\mathrm{NaHCO}_{3}$ and was extracted with $\mathrm{CH}_{2} \mathrm{Cl}_{2}$ again. The combined organics were dried, and concentrated in vacuo. The residue was purified by flash chromatography ( $n$-hexane/AcOEt $=1: 1)$ to afford 23a $(19.4 \mathrm{mg}, 80 \%)$ as a red solid. 
23a: $\mathrm{mp} 150^{\circ} \mathrm{C}$ decomp. IR (KBr): v 3391, 2926, 2851, 1661, 1572, 1524, 1487, $1460 \mathrm{~cm}^{-1} .{ }^{1} \mathrm{H}$ NMR $(300 \mathrm{MHz}): \delta 1.85(\mathrm{~d}, 1 \mathrm{H}, J=12.5 \mathrm{~Hz}), 1.94(\mathrm{dd}, 1 \mathrm{H}, J=13.0,2.0$ Hz), 2.40 (s, 3H), 2.64 (t, 2H, $J=7.5 \mathrm{~Hz}), 3.67$ (br s, 1H), 3.69-3.87 (m, 2H), 3.83 (s, 2H), $4.12(\mathrm{dt}, 1 \mathrm{H}, J=20.0,6.0 \mathrm{~Hz}), 4.22(\mathrm{~d}, 1 \mathrm{H}, J=12.5 \mathrm{~Hz}), 5.06(\mathrm{~d}, 1 \mathrm{H}, J=12.5 \mathrm{~Hz})$, 5.95 (br s, 1H), $6.07(\mathrm{~d}, 1 \mathrm{H}, J=10.0 \mathrm{~Hz}), 7.12(\mathrm{~d}, 1 \mathrm{H}, J=10.0 \mathrm{~Hz}), 7.31(\mathrm{~d}, 2 \mathrm{H}, J=8.5$ $\mathrm{Hz}), 7.46(\mathrm{~s}, 1 \mathrm{H}), 7.98(\mathrm{~d}, 2 \mathrm{H}, J=8.5 \mathrm{~Hz}) .{ }^{13} \mathrm{C} \mathrm{NMR}(125 \mathrm{MHz}): \delta 17.8,21.7,28.9$, $38.9,44.3,49.5,52.3,68.0,80.2,108.5,118.6,122.1,123.0,125.4,126.0,128.6,129.8$, $134.6,143.7,145.9,152.5,157.4,168.4,190.7 .[\alpha]_{\mathrm{D}}{ }^{26.2}-28.6^{\circ}\left(\mathrm{c} 0.281, \mathrm{CHCl}_{3}\right)$. HRFABMS calcd for $\mathrm{C}_{26} \mathrm{H}_{23} \mathrm{BrN}_{3} \mathrm{O}_{3} \mathrm{~S}\left(M+\mathrm{H}^{+}\right) ; 568.0541$, found 568.0549.

\section{3,5,7,8,9,10-hexahydro-2H-1,5,7-triaza-acephenanthrylen-6-one-8-}

\section{methylene[5']ether-10-spiro-4'-(2'-bromo)cyclohex-2'-en-1'-one (23b).}

To a stirred solution of desilylated 21 'a $(9.1 \mathrm{mg}, 0.0160 \mathrm{mmol})$ in $\mathrm{CH}_{2} \mathrm{Cl}_{2}(1.0 \mathrm{~mL})$ was added $30 \% \mathrm{HBr}-\mathrm{AcOH}(0.015 \mathrm{~mL})$ at $-78^{\circ} \mathrm{C}$ under a nitrogen atmosphere. The reaction mixture was warmed from $-78^{\circ} \mathrm{C}$ to $4^{\circ} \mathrm{C}$ by stirring for $41 \mathrm{~h}$ in a cooled environment $\left(4^{\circ} \mathrm{C}\right)$. The mixture was quenched with aqueous saturated $\mathrm{NaHCO}_{3}$ and extracted with $\mathrm{CH}_{2} \mathrm{Cl}_{2}$. The aqueous layer was treated with saturated aqueous $\mathrm{NaHCO}_{3}$ and was extracted with $\mathrm{CH}_{2} \mathrm{Cl}_{2}$ again. The combined organics were dried, and concentrated in vacuo. The residue was purified by flash chromatography ( $n$ hexane/AcOEt $=1: 1)$ to afford $\mathbf{2 3 b}(5.7 \mathrm{mg}, 63 \%)$ as a red solid.

23b: a red solid, $\mathrm{mp} 180^{\circ} \mathrm{C}$ decomp. IR (KBr): v 3393, 2926, 1682, 1659, 1595, 1572 , 1524, 1493, $1460 \mathrm{~cm}^{-1} .{ }^{1} \mathrm{H}$ NMR (300 MHz): $\delta 1.83$ (d, 1H, $\left.J=12.5 \mathrm{~Hz}\right), 2.06(\mathrm{~d}, 1 \mathrm{H}, J$ $=13.5 \mathrm{~Hz}), 2.42(\mathrm{~s}, 3 \mathrm{H}), 2.68(\mathrm{dd}, 2 \mathrm{H}, J=8.5,1.5 \mathrm{~Hz}), 2.85(\mathrm{dd}, 1 \mathrm{H}, J=16.5,5.5 \mathrm{~Hz})$, $3.03(\mathrm{dd}, 1 \mathrm{H}, J=16.5,13.0 \mathrm{~Hz}), 3.64$ (br s, $1 \mathrm{H}), 3.74$ (d, $1 \mathrm{H}, J=12.0 \mathrm{~Hz}), 3.80-3.89$ $(\mathrm{m}, 2 \mathrm{H}), 4.26(\mathrm{dt}, 1 \mathrm{H}, J=17.5,6.0 \mathrm{~Hz}), 4.34(\mathrm{dd}, 1 \mathrm{H}, J=11.5,5.5 \mathrm{~Hz}), 5.89$ (d, 1H, $J=$ $4.0 \mathrm{~Hz}), 7.33$ (d, 2H, $J=8.0 \mathrm{~Hz}), 7.49$ (s, 1H), 7.51 (s, 1H), 8.02 (d, 2H, $J=8.5 \mathrm{~Hz})$.

${ }^{13} \mathrm{C}$ NMR (75 MHz): $\delta 17.8,21.7,27.2,37.4,39.2,44.3,49.7,68.1,74.4,108.5,118.5$, 119.1, 122.0, 125.3, 125.9, 128.5, 128.6, 129.7, 129.8, 134.5, 143.3, 145.9, 152.3, 157.9, 
168.4, 190.2. $[\alpha]_{D}{ }^{27.2}-49.8^{\circ}$ (c $0.227, \mathrm{CHCl}_{3}$ ). HR-FABMS calcd for $\mathrm{C}_{26} \mathrm{H}_{23} \mathrm{BrN}_{3} \mathrm{O}_{3} \mathrm{~S}$ $\left(M+\mathrm{H}^{+}\right) ; 568.0542$, found 568.0556.

\section{8-Methoxy-3,5,7,9,10-hexahydro-2H-1,5,7-triazaacephenanthrylen-6-one-10-} spiro-4'-cyclohexa-2'-bromo-2',5'-diene-1'-one (24).

To a stirred solution of $22(19.9 \mathrm{mg}, 0.0350 \mathrm{mmol})$ in $\mathrm{CH}_{2} \mathrm{Cl}_{2}(2 \mathrm{~mL})-\mathrm{MeOH}(1.0$ $\mathrm{mL})$ was added $\mathrm{Pb}(\mathrm{OAc})_{4}(31.0 \mathrm{mg}, 0.0700 \mathrm{mmol})$ at $0^{\circ} \mathrm{C}$ under a nitrogen atmosphere. The reaction mixture was stirred for $1.5 \mathrm{~h}$ at $0^{\circ} \mathrm{C}$ then to room temperature. The mixture was then concentrated in vacuo. The residue was purified by flash chromatography ( $n$ hexane/AcOEt $\left./ \mathrm{Et}_{3} \mathrm{~N}=100: 100: 0.1\right)$ to give $\mathbf{2 4}(15.9 \mathrm{mg}, 80 \%)$ as a diastereomeric mixture $(\mathbf{2 4 a}+\mathbf{2 4 b})$.

24a: (less polar): a yellow solid. IR (KBr): $v$ 3385, 1660, 1585, 1530, 1495, 1460, $1375 \mathrm{~cm}^{-1} .{ }^{1} \mathrm{H}$ NMR (500 MHz): $\delta 1.92(\mathrm{~d}, 1 \mathrm{H}, J=14.0 \mathrm{~Hz}), 2.00(\mathrm{dd}, 1 \mathrm{H}, J=3.5,14.0$ $\mathrm{Hz}), 2.41$ (s, 3H), $2.62(\mathrm{t}, 2 \mathrm{H}, J=7.5 \mathrm{~Hz}), 3.30$ (s, 3H), $3.88(\mathrm{dt}, 1 \mathrm{H}, J=8.0,18.5 \mathrm{~Hz})$, 4.09 (dt, 1H, $J=7.5,18.5 \mathrm{~Hz}), 4.72(\mathrm{~m}, 1 \mathrm{H}), 6.23$ (d, 1H, $J=10.0 \mathrm{~Hz}), 6.49$ (br s, 1H), $7.20(\mathrm{~d}, 1 \mathrm{H}, J=3.0 \mathrm{~Hz}), 7.27(\mathrm{dd}, 1 \mathrm{H}, J=2.5,10.0 \mathrm{~Hz}), 7.32(\mathrm{~d}, 2 \mathrm{H}, J=8.5 \mathrm{~Hz}), 7.46$ $(\mathrm{s}, 1 \mathrm{H}), 8.01(\mathrm{~d}, 2 \mathrm{H}, J=8.5 \mathrm{~Hz}) .{ }^{13} \mathrm{C}$ NMR $(125 \mathrm{MHz}): \delta 17.4,21.7,37.7,42.9,49.9$, $54.5,79.3,106.9,118.5,121.9,122.7,125.1,125.4,126.3,128.6,129.8,134.6,139.2$, 145.9, 152.9, 153.4, 155.8, 168.3, 179.3. UV/Vis $\left(\mathrm{CH}_{2} \mathrm{Cl}_{2}\right): \lambda \max (\varepsilon)=327(11000), 229$ $\mathrm{nm}$ (28000). HR-FABMS calcd for $\mathrm{C}_{26} \mathrm{H}_{23} \mathrm{BrN}_{3} \mathrm{O}_{3} \mathrm{~S}\left(M+\mathrm{H}^{+}\right)$; 568.0542, found 568.0551.

24b: (polar): a yellow solid. IR (KBr): $v$ 3385, 1660, 1585, 1525, 1490, 1460, 1375 $\mathrm{cm}^{-1} .{ }^{1} \mathrm{H}$ NMR (500 MHz): $\delta 1.91(\mathrm{~d}, 1 \mathrm{H}, J=13.0 \mathrm{~Hz}), 1.98(\mathrm{dd}, 1 \mathrm{H}, J=3.0,14.0 \mathrm{~Hz})$, $2.41(\mathrm{~s}, 3 \mathrm{H}), 2.56-2.63(\mathrm{~m}, 2 \mathrm{H}), 3.13(\mathrm{~s}, 3 \mathrm{H}), 3.84(\mathrm{dt}, 1 \mathrm{H}, J=8.0,19.0 \mathrm{~Hz}), 4.07(\mathrm{dt}$, $1 \mathrm{H}, J=6.5,19.5 \mathrm{~Hz}), 4.72(\mathrm{~m}, 1 \mathrm{H}), 6.32(\mathrm{~d}, 1 \mathrm{H}, J=9.5 \mathrm{~Hz}), 6.51$ (br s, 1H), 6.81 (dd, $1 \mathrm{H}, J=3.0,10.0 \mathrm{~Hz}), 7.32(\mathrm{~d}, 2 \mathrm{H}, J=8.5 \mathrm{~Hz}), 7.46(\mathrm{~s}, 1 \mathrm{H}), 7.69(\mathrm{~d}, 1 \mathrm{H}, J=3.0 \mathrm{~Hz})$, $8.00(\mathrm{~d}, 2 \mathrm{H}, J=8.5 \mathrm{~Hz}) .{ }^{13} \mathrm{C} \mathrm{NMR}(125 \mathrm{MHz}): \delta 17.5,21.7,37.9,42.8,49.8,54.5,79.4$, $106.8,118.5,121.7,122.9,125.4,125.5,126.3$, 128.6, 129.8, 134.5, 139.4, 145.9, 153.0, 153.8, 155.7, 168.4, 179.2. UV/Vis $\left(\mathrm{CH}_{2} \mathrm{Cl}_{2}\right): \lambda \max (\varepsilon)=329(12000), 232 \mathrm{~nm}(26000)$. HR-FABMS calcd for $\mathrm{C}_{26} \mathrm{H}_{23} \mathrm{BrN}_{3} \mathrm{O}_{3} \mathrm{~S}\left(M+\mathrm{H}^{+}\right)$; 568.0542, found 568.0526. 
25b: unstable diastereomeric mixture (25b-I + 25b-II). HR-FABMS Calcd for $\mathrm{C}_{33} \mathrm{H}_{29} \mathrm{BrN}_{3} \mathrm{O}_{3} \mathrm{~S}_{2}\left(M+\mathrm{H}^{+}\right) ; 690.0732$, found 690.0715.

\section{3-(Toluene-4-sulfonyl)-discorhabdin A (5-(Toluene-4-sulfonyl)-3,5,7,8,9,10-} hexahydro-2H-1,5,7-triaza-acephenanthrylen-6-one-8-sulfanyl[5']-10-spiro-4'-(2'bromo)-cyclohex-2'-en-1'-one) (26).

To a stirred solution of $\mathbf{2 4}$ (diastereomeric mixture: 24a $+\mathbf{2 4 b})(25.0 \mathrm{mg}, 0.044$ $\mathrm{mmol})$ in $\mathrm{CH}_{2} \mathrm{Cl}_{2}(1.5 \mathrm{~mL})$ was added 4-methoxybenzylthiol $(7.4 \mu \mathrm{L}, 0.053 \mathrm{mmol})$ followed by dropwise $30 \% \mathrm{HBr}-\mathrm{AcOH}(0.038 \mathrm{~mL})$ at $-78^{\circ} \mathrm{C}$ under a nitrogen atmosphere. The reaction mixture was warmed from $-78^{\circ} \mathrm{C}$ to $4^{\circ} \mathrm{C}$ by stirring for $38 \mathrm{~h}$ in a cooled environment $\left(4^{\circ} \mathrm{C}\right)$. The mixture was quenched with $40 \%$ aqueous $\mathrm{MeNH}_{2}$, diluted with $\mathrm{CH}_{2} \mathrm{Cl}_{2}$, acidified with $10 \%$ aqueous $\mathrm{HCl}$, and then extracted with $\mathrm{CH}_{2} \mathrm{Cl}_{2}$. The aqueous layer was treated with saturated aqueous $\mathrm{NaHCO}_{3}$ and was extracted with $\mathrm{CH}_{2} \mathrm{Cl}_{2}$ again. The combined organics were dried, and concentrated in vacuo. The residue was purified by preparative TLC $\left(\mathrm{CH}_{2} \mathrm{Cl}_{2} / \mathrm{MeOH}=30 / 1\right)$ to produce $26(5.4 \mathrm{mg}$, $22 \%,>99 \%$ ee $(\mathbf{2 6} \cdot \mathrm{HCl}$ salt by HPLC (DAICEL CHIRALCEL OD, flow rate 1.0 $\mathrm{mL} / \mathrm{min}, n$-hexane/ $i$-PrOH 95/5, $20^{\circ} \mathrm{C}$ )), 5-(toluene-4-sulfonyl)-3,5,7,8,9,10-hexahydro2H-1,5,7-triaza-acephenanthrylen-6-one-8-sulfanyl-[5']-10-spiro-4'-cyclohex-2'-en-1'one (28) (6.9 mg, 32\%), and a trace amount of 5-(toluene-4-sulfonyl)-3,5,7,8,9,10hexahydro-2H-1,5,7-triaza-acephenanthrylen-6-one-8-sulfanyl[3']-10-spiro-4'-(2'bromo)-cyclohex-5'-en-1'-one (27) respectively.

26: a red solid. $\mathrm{mp}>300^{\circ} \mathrm{C}$. IR $(\mathrm{KBr}): \vee 3365,1680,1660,1610,1595,1565,1525$, 1455, $1375 \mathrm{~cm}^{-1} .{ }^{1} \mathrm{H}$ NMR (300 MHz): $\delta 2.42$ (s, 3H), 2.61-2.77 (m, 4H), 2.80-2.85 (m, 2H), $3.88(\mathrm{dt}, 1 \mathrm{H}, J=9.0,18.5 \mathrm{~Hz}), 4.29(\mathrm{dt}, 1 \mathrm{H}, J=18.5,6.0 \mathrm{~Hz}), 4.62(\mathrm{dd}, 1 \mathrm{H}, J=$ 8.0, $11.5 \mathrm{~Hz}), 5.28$ (t, 1H, $J=4.0 \mathrm{~Hz}), 5.93$ (s, 1H), 7.33 (d, 2H, J = 8.5 Hz), 7.43 (s, $1 \mathrm{H}), 7.49(\mathrm{~s}, 1 \mathrm{H}), 8.02(\mathrm{~d}, 2 \mathrm{H}, J=8.5 \mathrm{~Hz}) .{ }^{13} \mathrm{C} \mathrm{NMR}(125 \mathrm{MHz}): \delta 17.7,21.7,39.9$, $45.5,50.0,50.0,55.9,61.3,116.2,118.4,119.6,121.9,125.1,126.1,128.7,129.8$, $134.5,140.6,146.0,152.8,154.8,168.3,188.0 . \mathrm{UV} / \mathrm{Vis}\left(\mathrm{CHCl}_{3}\right): \lambda \max (\varepsilon)=483(1400)$, 
326 (12200), 265 (14400), $242 \mathrm{~nm}(18200) .[\alpha]_{\mathrm{D}}^{24.9}-72.7^{\circ}$ (c $\left.0.0525, \mathrm{CHCl}_{3}\right) . \mathrm{HR}-$ FABMS calcd for $\mathrm{C}_{25} \mathrm{H}_{21} \mathrm{BrN}_{3} \mathrm{O}_{4} \mathrm{~S}_{2}\left(M+\mathrm{H}^{+}\right) ; 570.0157$, found 570.0171 .

27: a red solid. $\mathrm{mp}>300^{\circ} \mathrm{C}$. IR $(\mathrm{KBr}): \vee 3375,1690,1660,1615,1595,1560,1525$, 1480, 1460, $1380 \mathrm{~cm}^{-1} .{ }^{1} \mathrm{H}$ NMR (300 MHz): $\delta 2.42$ (s, 3H), 2.63-2.71 (m, 4H), 3.77$3.87(\mathrm{~m}, 1 \mathrm{H}), 4.23$ (dt, 1H, $J=17.5,6.5 \mathrm{~Hz}), 4.77-4.86(\mathrm{~m}, 2 \mathrm{H}), 5.30$ (br s, 1H), 5.99 (d, $1 \mathrm{H}, J=4.5 \mathrm{~Hz}), 6.10(\mathrm{~d}, 1 \mathrm{H}, J=10.0 \mathrm{~Hz}), 7.07(\mathrm{~d}, 1 \mathrm{H}, J=10.5 \mathrm{~Hz}), 7.33(\mathrm{~d}, 2 \mathrm{H}, J=$ $8.5 \mathrm{~Hz}), 7.49(\mathrm{~s}, 1 \mathrm{H}), 8.01(\mathrm{~d}, 2 \mathrm{H}, J=8.5 \mathrm{~Hz}) .{ }^{13} \mathrm{C} \mathrm{NMR}(75 \mathrm{MHz}): \delta 17.7,21.7,41.5$, $49.5,49.6,60.6,61.4,66.6,115.0,118.4,121.8,123.5,125.2,126.0,128.7,129.8$, $134.4,140.5,146.0,152.9,154.5,168.2,188.6$. UV/Vis $\left(\mathrm{CH}_{2} \mathrm{Cl}_{2}\right): \lambda \max (\varepsilon)=489$ (1500), 327 (13300), $237 \mathrm{~nm}(27300)$. $[\alpha]_{\mathrm{D}}{ }^{24.3}+55.2^{\circ}$ (c $\left.0.0755, \mathrm{CHCl}_{3}\right)$. HR-FABMS calcd for $\mathrm{C}_{25} \mathrm{H}_{21} \mathrm{BrN}_{3} \mathrm{O}_{4} \mathrm{~S}_{2}\left(M+\mathrm{H}^{+}\right) ; 570.0157$, found 570.0142 .

28: a red solid. $\mathrm{mp}>300^{\circ} \mathrm{C} .{ }^{1} \mathrm{H}$ NMR $(300 \mathrm{MHz}): \delta 2.42(\mathrm{~s}, 3 \mathrm{H}), 2.57-2.78(\mathrm{~m}, 6 \mathrm{H})$, 3.75-3.88 (m, 1H), $4.26(\mathrm{dt}, 1 \mathrm{H}, J=17.5,6.5 \mathrm{~Hz}), 4.60(\mathrm{dd}, 1 \mathrm{H}, J=11.5,7.0 \mathrm{~Hz}), 5.26$ $(\mathrm{t}, 1 \mathrm{H}, J=3.0 \mathrm{~Hz}), 5.90(\mathrm{~d}, 1 \mathrm{H}, J=4.5 \mathrm{~Hz}), 5.99(\mathrm{~d}, 1 \mathrm{H}, J=10.0 \mathrm{~Hz}), 7.03(\mathrm{~d}, 1 \mathrm{H}, J=$ $10.0 \mathrm{~Hz}), 7.33(\mathrm{~d}, 2 \mathrm{H}, J=8.0 \mathrm{~Hz}), 7.49(\mathrm{~s}, 1 \mathrm{H}), 8.03(\mathrm{~d}, 2 \mathrm{H}, J=8.5 \mathrm{~Hz}) .{ }^{13} \mathrm{C}$ NMR $(75$ MHz): $\delta$ 17.7, 21.7, 41.0, 45.9, 47.3, 49.7, 56.3, 61.0, 116.9, 118.4, 121.9, 125.4, 125.8, $128.7,129.8,134.5,140.8,145.9,153.1,154.6,168.5,196.2$. UV/Vis $\left(\mathrm{CHCl}_{3}\right): \lambda \max$ $(\varepsilon)=479(1500), 327(13000), 243 \mathrm{~nm}(22500) .[\alpha]_{\mathrm{D}}^{22.4}+134^{\circ}\left(\mathrm{c} 0.146, \mathrm{CHCl}_{3}\right)$. HRFABMS calcd for $\mathrm{C}_{25} \mathrm{H}_{22} \mathrm{~N}_{3} \mathrm{O}_{4} \mathrm{~S}_{2}\left(M+\mathrm{H}^{+}\right) ; 492.1052$, found 492.1049 .

\section{Discorhabdin A (1).}

To a stirred solution of 15 (3.9 mg, $0.00684 \mathrm{mmol})$ in THF $(0.7 \mathrm{~mL})$ was added 0.2 $\mathrm{M} \mathrm{NaOMe} / \mathrm{MeOH}(0.09 \mathrm{~mL})$ at $0^{\circ} \mathrm{C}$ under a nitrogen atmosphere. The reaction mixture was stirred for $5 \mathrm{~h}$ at $0{ }^{\circ} \mathrm{C}$. The mixture was purified by preparative TLC $\left(\mathrm{CH}_{2} \mathrm{Cl}_{2} / \mathrm{MeOH}=10 / 1\right)$ to give $1(1.7 \mathrm{mg}, 61 \%)$ as its free base. $0.1 \mathrm{~N} \mathrm{HCl} / \mathrm{MeOH}(0.05$ $\mathrm{mL})$ was added dropwise to a stirred solution of $\mathbf{1}$ in $\mathrm{MeOH}(0.5 \mathrm{~mL})$ at room temperature, and the mixture was concentrated in vacuo to give $\mathrm{HCl}$ salt of $\mathbf{1}(\mathbf{1} \cdot \mathbf{H C l})$, which is more stable than its free base form, as a green solid. 
1•HCl: IR (KBr): v 3650-2400, 1680, 1610, 1580, 1525, 1435, 1410, $1390 \mathrm{~cm}^{-1} .{ }^{1} \mathrm{H}$ NMR (300 MHz, $\left.\mathrm{CD}_{3} \mathrm{OD}\right): \delta 2.60(\mathrm{dd}, 1 \mathrm{H}, J=12.5,1.0 \mathrm{~Hz}), 2.88(\mathrm{dd}, 1 \mathrm{H}, J=17.0,6.5$ $\mathrm{Hz}), 2.94-3.02(\mathrm{~m}, 3 \mathrm{H}), 3.07$ (dd, $1 \mathrm{H}, J=17.0,12.0 \mathrm{~Hz}), 3.80(\mathrm{dt}, 1 \mathrm{H}, J=13.5,5.0 \mathrm{~Hz})$, $3.93(\mathrm{dt}, 1 \mathrm{H}, J=13.5,6.5 \mathrm{~Hz}), 4.51(\mathrm{dd}, 1 \mathrm{H}, J=12.0,6.5 \mathrm{~Hz}), 5.37$ (dd, $1 \mathrm{H}, J=4.0$, $1.0 \mathrm{~Hz}), 7.19$ (s, 1H), 7.57 (s, 1H). ${ }^{13} \mathrm{C}$ NMR (125 MHz, [D 6 ]DMSO): $\delta$ 18.4, 40.8, 44.2, 44.8, 49.9, 54.2, 59.1, 104.2, 120.3, 123.6, 123.7, 126.1, 127.6, 148.3, 150.7, 154.1, 166.3, 187.2. UV/Vis $(\mathrm{MeOH}): \lambda \max (\varepsilon)=558(800), 349(7000), 249 \mathrm{~nm}(17100)$. $[\alpha]_{\mathrm{D}}{ }^{24.4}+388^{\circ}\left(\mathrm{c} 0.06, \mathrm{CD}_{3} \mathrm{OD}\right) .\left([\alpha]_{\mathrm{D}}+400^{\circ}\left(\mathrm{c} 0.05, \mathrm{CH}_{3} \mathrm{OH}\right)^{1 \mathrm{c}}\right)$; HR-FABMS calcd for $\mathrm{C}_{18} \mathrm{H}_{15} \mathrm{BrN}_{3} \mathrm{O}_{2} \mathrm{~S}\left(M+\mathrm{H}^{+}\right) ; 416.0068$, found 416.0066.

\section{7-[2-(3-Bromo-4-tert-butyldimethylsilyloxyphenyl)-1-(tert-butyldimethyl-} silyloxymethyl)ethylamino]-1-(toluene-4-sulfonyl)-3,4-dihydro-1H-pyrrolo[4,3,2de]quinonlin-8-one (20e).

$0.1 \mathrm{~N} \mathrm{MeOH}$ solution of $\mathrm{HCl}(2.5 \mathrm{~mL})$ was added to $19 \mathrm{a}(118.1 \mathrm{mg}, 0.165 \mathrm{mmol})$ at room temperature under a nitrogen atmosphere and the mixture was stirred for $30 \mathrm{~min}$. The above mixture was added dropwise to a stirred solution of $\mathbf{1 6 b}(58.7 \mathrm{mg}, 0.165$ $\mathrm{mmol})$ in $\mathrm{MeOH}(4.0 \mathrm{~mL})$ at room temperature under a nitrogen atmosphere. The mixture was stirred for $48 \mathrm{~h}$ under the same conditions and was then concentrated in vacuo. The residue was purified by flash chromatography (n-hexane $\left./ \mathrm{AcOEt} / \mathrm{Et}_{3} \mathrm{~N}=100: 100: 0.1\right)$ to give $20 \mathrm{e}(88.7 \mathrm{mg}, 67 \%)$ as a red oil.

20e: IR (KBr): v 2930, 2953, 2856, 1661, 1578, 1531, $1493 \mathrm{~cm}^{-1} .{ }^{1} \mathrm{H}$ NMR (500 MHz, $\left.\mathrm{CD}_{3} \mathrm{OD}\right): \delta 0.02(\mathrm{~s}, 6 \mathrm{H}), 0.14(\mathrm{~s}, 3 \mathrm{H}), 0.16(\mathrm{~s}, 3 \mathrm{H}), 0.90(\mathrm{~s}, 9 \mathrm{H}), 0.99(\mathrm{~s}, 9 \mathrm{H}), 2.41$ (s, $3 \mathrm{H}), 2.73-2.77(\mathrm{~m}, 3 \mathrm{H}), 2.82(\mathrm{dd}, 1 \mathrm{H}, J=13.0,6.5 \mathrm{~Hz}), 3.58-3.61(\mathrm{~m}, 2 \mathrm{H}), 3.66(\mathrm{dd}, 1 \mathrm{H}$, $J=9.5,3.5 \mathrm{~Hz}$ ), 3.97 (br s, 2H), 5.47 (br s, 1H), 6.77 (d, 1H, $J=8.5 \mathrm{~Hz}), 7.05$ (dd, 1H, $J=8.0,2.0 \mathrm{~Hz}), 7.36(\mathrm{~d}, 1 \mathrm{H}, J=2.5 \mathrm{~Hz}), 7.38(\mathrm{~d}, 2 \mathrm{H}, J=8.0 \mathrm{~Hz}), 7.63(\mathrm{br} \mathrm{s}, 1 \mathrm{H}), 8.01$ $(\mathrm{d}, 2 \mathrm{H}, J=8.5 \mathrm{~Hz})$. HR-FABMS calcd for $\mathrm{C}_{38} \mathrm{H}_{33} \mathrm{BrN}_{3} \mathrm{O}_{3} \mathrm{SSi}_{2}\left(M+\mathrm{H}^{+}\right) ; 798.2428$, found 798.2418. This compound gradually decomposed during ${ }^{13} \mathrm{C}$ NMR measurement due to its unstability. 


\section{5-(Toluene-4-sulfonyl)-3,5,7,8,9,10-hexahydro-2H-1,5,7-triaza-}

acephenanthrylen-6-one-8-(tert-butyldimethylsilyloxymethyl)-10-spiro-4'-(2'bromo)-cyclohexa-2',5'-dien-1'-one (21a, 21'a).

To a stirred solution of 20e (43.4 mg, $0.0543 \mathrm{mmol})$ in $\mathrm{CF}_{3} \mathrm{CH}_{2} \mathrm{OH}(1.5 \mathrm{~mL})$ was added MK10 (31.9 mg) and PIFA (93.4 mg, $0.217 \mathrm{mmol}$ ) sequentially at room temperature. The mixture was stirred for $7 \mathrm{~h}$. under the same conditions and then concentrated in vacuo. The residue was purified by flash chromatography ( $n$ hexane/AcOEt $\left./ \mathrm{Et}_{3} \mathrm{~N}=100: 50: 0.1\right)$ to afford two diastereomers 21a (13.7 mg, 37.2\%) and 21'a (2.9 mg, 7.8\%) respectively as a red amorphous. The ratio of 21a and 21'a was determined by HPLC (CHIRALPAK AD-H, flow rate $1.0 \mathrm{~mL} / \mathrm{min}, n$-hexane/ $i$ $\left.\operatorname{PrOH}=95 / 5,25^{\circ} \mathrm{C}\right)$.

\section{7-[2-(3-Bromo-4-hydroxyphenyl)-1-(triisopropylsilyloxymethyl)ethylamino]-1-}

\section{(toluene-4-sulfonyl)-3,4-dihydro-1H-pyrrolo[4,3,2-de]quinonlin-8-one (20b).}

To a stirred solution of monobrominated $N$-tritylated $(L)$-tyrosinol $(1.5 \mathrm{~g}, 3.08 \mathrm{mmol})$ in $\mathrm{CH}_{2} \mathrm{Cl}_{2}(60 \mathrm{~mL})$ was added $\mathrm{DBU}(1.98 \mathrm{~mL}, 9.24 \mathrm{mmol})$ and triisopropylsilyl chloride (TIPSCl) $(2.31 \mathrm{~mL}, 15.4 \mathrm{mmol})$ sequentially at $0^{\circ} \mathrm{C}$ under a nitrogen atmosphere. The reaction mixture was stirred for $0.75 \mathrm{~h}$ at $0^{\circ} \mathrm{C}$. The mixture was then quenched with aqueous saturated $\mathrm{NaHCO}_{3}$ and extracted with $\mathrm{CH}_{2} \mathrm{Cl}_{2}$. The combined organics were washed with brine, dried, and concentrated in vacuo. The residue was purified by flash chromatography ( $n$-hexane/AcOEt=20/1) to give [2-[3-bromo-4-(triisopropylsilyloxy)phenyl]-1-(triisopropylsilyloxymethyl)ethyl]tritylamine (2.47 g, quant) as a colorless oil.

IR (KBr): v 3057, 3030, 2943, 2866, 1599, 1493, $1462 \mathrm{~cm}^{-1} .{ }^{1} \mathrm{H}$ NMR (300 MHz): $\delta$ $0.96(\mathrm{~s}, 18 \mathrm{H}), 1.06$ (s, 6H), 1.09 (s, 12H), 0.91-1.09 (m, 3H), 1.19-1.32 (m, 3H), 2.332.39 (m, 2H), $2.66(\mathrm{~m}, 1 \mathrm{H}), 3.05$ (dd, 1H, $J=9.5,5.5 \mathrm{~Hz}), 3.20(\mathrm{dd}, 1 \mathrm{H}, J=9.5,4.5$ $\mathrm{Hz}), 6.67(\mathrm{~d}, 1 \mathrm{H}, J=8.5 \mathrm{~Hz}), 6.71(\mathrm{dd}, 1 \mathrm{H}, J=8.0,1.5 \mathrm{~Hz}), 7.12-7.26(\mathrm{~m}, 10 \mathrm{H}), 7.54$ $(\mathrm{d}, 6 \mathrm{H}, J=7.5 \mathrm{~Hz}) .{ }^{13} \mathrm{C}$ NMR $(75 \mathrm{MHz}): \delta 11.9,12.9,18.0,18.1,36.7,55.6,64.3,71.0$, 114.6, 119.1, 126.3, 127.8, 128.8, 129.2, 133.2, 134.3, 147.1, 150.9. HR-FABMS calcd for $\mathrm{C}_{46} \mathrm{H}_{67} \mathrm{BrNO}_{2} \mathrm{Si}_{2}\left(\mathrm{M}+\mathrm{H}^{+}\right) ; 800.3894$, found 800.3898.

To a stirred solution of [2-[3-bromo-4-(triisopropylsilyloxy)phenyl]-1-(triisopropyl- 
silyloxymethyl)ethyl]tritylamine $(2.53 \mathrm{~g}, 3.16 \mathrm{mmol})$ in THF $(125 \mathrm{~mL})$ was added TBAF $\left(3.16 \mathrm{~mL}(1.0 \mathrm{M}\right.$ solution in THF), $3.16 \mathrm{mmol})$ at $0^{\circ} \mathrm{C}$ under a nitrogen atmosphere. The reaction mixture was stirred for $0.5 \mathrm{~h}$ at $0^{\circ} \mathrm{C}$. The mixture was then quenched with aqueous saturated $\mathrm{NH}_{4} \mathrm{Cl}$ and extracted with AcOEt. The combined organics were washed with brine, dried, and concentrated in vacuo. The residue was purified by flash chromatography ( $n$-hexane/AcOEt=10/1) to give [2-[3-bromo-4hydroxyphenyl]-1-(triisopropylsilyloxymethyl)ethyl]tritylamine $(1.83 \mathrm{~g}, 90 \%)$ as a colorless oil.

IR (KBr): v 2941, 2864, 1493, 1462, $1448 \mathrm{~cm}^{-1} .{ }^{1} \mathrm{H}$ NMR (300 MHz): $\delta 0.97$ (s, 18H), 0.93-1.01 (m, 3H), 2.32-2.45 (m, 2H), $2.70(\mathrm{~m}, 1 \mathrm{H}), 3.02(\mathrm{dd}, 1 \mathrm{H}, J=9.5,5.5 \mathrm{~Hz}), 3.18$ (dd, $1 \mathrm{H}, J=9.5,4.0 \mathrm{~Hz}), 5.38($ br s, 2H), $6.80(\mathrm{~s}, 2 \mathrm{H}), 7.09$ (s, 1H), 7.13-7.26 (m, 9H), $7.54(\mathrm{~d}, 6 \mathrm{H}, J=7.5 \mathrm{~Hz}) .{ }^{13} \mathrm{C}$ NMR $(75 \mathrm{MHz}): \delta 11.9,17.7,36.6,55.4,64.1,71.0,109.7$, 115.4, 126.3, 127.8, 128.7, 130.3, 132.9, 133.0, 147.1, 150.3. HR-FABMS calcd for $\mathrm{C}_{37} \mathrm{H}_{47} \mathrm{BrNO}_{2} \mathrm{Si}\left(\mathrm{M}+\mathrm{H}^{+}\right) ; 644.2559$, found 644.2583 .

$0.1 \mathrm{~N} \mathrm{MeOH}$ solution of $\mathrm{HCl}(1.0 \mathrm{~mL})$ was added to [2-[3-bromo-4-hydroxyphenyl]1-(triisopropylsilyloxymethyl)ethyl]tritylamine $(37.1 \mathrm{mg}, 0.0575 \mathrm{mmol})$ at room temperature under a nitrogen atmosphere and the mixture was stirred for $30 \mathrm{~min}$. The above mixture was added dropwise to a stirred solution of $\mathbf{1 6 b}(20.5 \mathrm{mg}, 0.0575 \mathrm{mmol})$ in $\mathrm{MeOH}(1.0 \mathrm{~mL})$ at room temperature under a nitrogen atmosphere. The mixture was stirred for $24 \mathrm{~h}$ under the same conditions and was then concentrated in vacuo. The residue was purified by flash chromatography $\left(\mathrm{CH}_{2} \mathrm{Cl}_{2} / \mathrm{MeOH} / \mathrm{Et}_{3} \mathrm{~N}=100: 5: 0.1\right)$ to give 20b (23.0 mg, 55\%) as a red amorphous solid.

IR (KBr): v 2941, 2864, 1666, 1612, 1556, $1537 \mathrm{~cm}^{-1} .{ }^{1} \mathrm{H}$ NMR (500 MHz, $\left.\mathrm{CD}_{3} \mathrm{OD}\right): \delta 1.01-1.11(\mathrm{~m}, 21 \mathrm{H}), 2.42(\mathrm{~s}, 3 \mathrm{H}), 2.79(\mathrm{t}, 2 \mathrm{H}, J=8.0 \mathrm{~Hz}), 2.76-2.89(\mathrm{~m}$, 2H), 3.59-3.63 (m, 1H), 3.78 (dd. 1H, $J=10.0,4.0 \mathrm{~Hz}), 3.97$ (t, 2H, $J=7.5 \mathrm{~Hz}), 5.51$ (br s, 1H), 6.77 (d, 1H, $J=7.5 \mathrm{~Hz}), 7.02(\mathrm{dd} .1 \mathrm{H}, J=8.0,2.0 \mathrm{~Hz}), 7.31$ (d, 1H, $J=2.0$ $\mathrm{Hz}), 7.39$ (d, 1H, $J=8.0 \mathrm{~Hz}), 7.68$ (br s, $1 \mathrm{H}), 8.01$ (d, 2H, $J=8.5 \mathrm{~Hz}) .{ }^{13} \mathrm{C}$ NMR $(75$ $\left.\mathrm{MHz}, \mathrm{CD}_{3} \mathrm{OD}\right): \delta 9.19,9.30,13.0,18.4,21.8,36.2,43.6,47.1,59.3,64.9,86.9,110.7$, 
$117.4,120.0,124.0,129.1,130.1,130.4,130.7,130.9,131.2,134.5,135.0,148.6$, 154.3, 157.8, 166.9. HR-FABMS calcd for $\mathrm{C}_{33} \mathrm{H}_{43} \mathrm{BrN}_{3} \mathrm{O}_{3} \mathrm{SSi}\left(M+\mathrm{H}^{+}\right) ; 726.2033$, found 726.2029 .

\section{5-(Toluene-4-sulfonyl)-3,5,7,8,9,10-hexahydro-2H-1,5,7-triaza-}

\section{acephenanthrylen-6-one-8-(triisopropylsilyloxymethyl)-10-spiro-4'-(2'-bromo)- cyclohexa-2',5'-dien-1'-one (21b, 21'b).}

To a stirred solution of $\mathbf{2 0 b}(9.2 \mathrm{mg}, 0.013 \mathrm{mmol})$ in $\mathrm{CF}_{3} \mathrm{CH}_{2} \mathrm{OH}(0.8 \mathrm{~mL})$ was added MK10 (4.0 mg) and PIFA (6.7 mg, $0.016 \mathrm{mmol})$ sequentially at room temperature. The mixture was stirred for $45 \mathrm{~min}$ under the same conditions and then concentrated in vacuo. The residue was purified by flash chromatography ( $n$-hexane/AcOEt/Et $3 \mathrm{~N}=$ 100:50:0.1) to afford two diastereomers $21 \mathbf{b}(2.0 \mathrm{mg}, 22 \%)$ and 21'b (1.5 $\mathrm{mg}, 15 \%)$ respectively as a red amorphous solid.

21b (less polar): IR (KBr): v 2943, 2864, 1659, 1574, 1528, 1479, $1462 \mathrm{~cm}^{-1} .{ }^{1} \mathrm{H}$ NMR (500 MHz): $\delta 1.04-1.25(\mathrm{~s}, 21 \mathrm{H}), 1.59(\mathrm{~d}, 1 \mathrm{H}, J=17.0 \mathrm{~Hz}), 1.73(\mathrm{t}, 1 \mathrm{H}, J=11.5$ $\mathrm{Hz}), 2.40$ (s, 3H), 2.60 (t, 2H, $J=7.0 \mathrm{~Hz}), 3.55-3.61(\mathrm{~m}, 2 \mathrm{H}), 3.80-3.86(\mathrm{~m}, 2 \mathrm{H}), 4.07$ (dt, 1H, $J=17.5,7.5 \mathrm{~Hz}), 6.15$ (br s, 1H), 6.27 (d, 1H, $J=10.0 \mathrm{~Hz}), 6.97$ (dd, 1H, $J=$ 9.5, 3.5 Hz), 7.27 (d, 1H, $J=2.5 \mathrm{~Hz}), 7.29$ (d, 1H, $J=8.5 \mathrm{~Hz}), 7.43(\mathrm{~s}, 1 \mathrm{H}), 8.02$ (d, 2H, $J=8.0 \mathrm{~Hz}) .{ }^{13} \mathrm{C}$ NMR $(125 \mathrm{MHz}): \delta 11.9,17.5,18.0,21.7,36.7,43.9,49.7,66.6,104.1$, $118.5,122.0,125.4,125.9,126.0,128.8,129.7,134.7,142.0,145.7,152.8,152.7,157.1$, 168.4, 179.0. UV/Vis $\left(\mathrm{CHCl}_{3}\right): \lambda \max (\varepsilon)=487$ (2300), 329 (12400), $235 \mathrm{~nm}$ (30200). HR-FABMS calcd for $\mathrm{C}_{33} \mathrm{H}_{43} \mathrm{BrN}_{3} \mathrm{O}_{3} \mathrm{SSi}\left(M+\mathrm{H}^{+}\right) ; 724.1876$, found 724.1888.

21'b (polar): IR (KBr): v 2943, 2864, 1661, 1574, 1528, 1479, $1462 \mathrm{~cm}^{-1} .{ }^{1} \mathrm{H}$ NMR (500 MHz): $\delta 1.01-1.17(\mathrm{~s}, 21 \mathrm{H}), 1.59(\mathrm{~d}, 1 \mathrm{H}, J=13.0 \mathrm{~Hz}), 1.73(\mathrm{t}, 1 \mathrm{H}, J=11.5 \mathrm{~Hz})$, $2.40(\mathrm{~s}, 3 \mathrm{H}), 2.54-2.64(\mathrm{~m}, 2 \mathrm{H}), 3.56-3.61(\mathrm{~m}, 2 \mathrm{H}), 3.76-3.84(\mathrm{~m}, 2 \mathrm{H}), 4.04(\mathrm{dt}, 1 \mathrm{H}, J=$ 18.0, $7.0 \mathrm{~Hz}), 6.18$ (br s, 1H), 6.31 (d, 1H, $J=9.5 \mathrm{~Hz}), 6.89$ (dd, 1H, $J=10.0,2.5 \mathrm{~Hz}$ ), $7.29(\mathrm{~d}, 1 \mathrm{H}, J=8.5 \mathrm{~Hz}), 7.41(\mathrm{~d}, 1 \mathrm{H}, J=2.5 \mathrm{~Hz}), 7.43(\mathrm{~s}, 1 \mathrm{H}), 8.02(\mathrm{~d}, 2 \mathrm{H}, J=8.5 \mathrm{~Hz})$. ${ }^{13} \mathrm{C}$ NMR (125 MHz): $\delta 11.9,17.6,18.0,21.7,36.9,44.1,49.5,49.7,66.5,104.1,118.5$, $121.9,123.9,125.0,125.5,126.0,128.8,129.7,134.7,142.1,145.7,152.3,152.9,157.1$, 
168.4, 179.0. UV/Vis $\left(\mathrm{CHCl}_{3}\right): \lambda \max (\varepsilon)=484$ (2300), 331 (13000), $232 \mathrm{~nm}(29000)$. HR-FABMS calcd for $\mathrm{C}_{33} \mathrm{H}_{43} \mathrm{BrN}_{3} \mathrm{O}_{3} \mathrm{SSi}\left(M+\mathrm{H}^{+}\right)$; 724.1876, found 724.1887.

\section{7-[2-(3-Bromo-4-hydroxyphenyl)-1-(tert-butyldiphenylsilyloxymethyl)} ethylamino]-1-(toluene-4-sulfonyl)-3,4-dihydro-1H-pyrrolo[4,3,2-de]quinonlin-8one (20c).

To a stirred solution of monobrominated $N$-tritylated $(L)$-tyrosinol $(177.1 \mathrm{mg}, 0.363$ mmol $)$ in $\mathrm{CH}_{2} \mathrm{Cl}_{2}(2.5 \mathrm{~mL})$ was added DBU $(0.27 \mathrm{~mL}, 1.82 \mathrm{mmol})$ and tertbutyldiphenylsilyl chloride (TBDPSCl) $(0.28 \mathrm{~mL}, 1.09 \mathrm{mmol})$ sequentially at $0^{\circ} \mathrm{C}$ under a nitrogen atmosphere. The reaction mixture was stirred for $2 \mathrm{~h}$ at $0^{\circ} \mathrm{C}$. The mixture was then quenched with aqueous saturated $\mathrm{NaHCO}_{3}$ and extracted with $\mathrm{CH}_{2} \mathrm{Cl}_{2}$. The combined organics were washed with brine, dried, and concentrated in vacuo. The residue was purified by flash chromatography ( $n$-hexane/AcOEt=30/1) to give [2-[3bromo-4-(tert-butyldiphenylsilyloxy)phenyl]-1-(tert-

butyldiphenylsilyloxymethyl)ethyl]tritylamine (160.3 mg, 46\%) as a colorless oil.

IR (KBr): v 3069, 2930, 2856, 1493, 1472, 1448, $1427 \mathrm{~cm}^{-1} .{ }^{1} \mathrm{H}$ NMR (300 MHz): $\delta$ 1.10 (s, 9H), 1.19 (s, 9H), 2.21 (dd, 1H, $J=13.0,4.0 \mathrm{~Hz}), 2.39$ (dd, 1H, $J=13.0,9.0$ $\mathrm{Hz}), 2.70(\mathrm{~m}, 1 \mathrm{H}), 3.18(\mathrm{dd}, 1 \mathrm{H}, J=9.0,4.0 \mathrm{~Hz}), 3.29(\mathrm{dd}, 1 \mathrm{H}, J=10.0,3.5 \mathrm{~Hz}), 6.28$ $(\mathrm{d}, 1 \mathrm{H}, J=8.5 \mathrm{~Hz}), 6.34(\mathrm{~d}, 1 \mathrm{H}, J=8.5 \mathrm{~Hz}), 7.18-7.77(\mathrm{~m}, 36 \mathrm{H}) .{ }^{13} \mathrm{C}$ NMR $(75 \mathrm{MHz})$ : $\delta 19.3,19.7,26.5,27.0,36.9,55.4,64.4,71.1,114.1,119.1,126.2,127.6,127.7,127.8$, $128.7,128.8,129.5,129.6,130.0,132.4,133.3,133.6,134.1,135.5,135.6,135.7,146.9$, 150.3. HR-FABMS calcd for $\mathrm{C}_{60} \mathrm{H}_{67} \mathrm{BrNO}_{2} \mathrm{Si}_{2} \mathrm{Na}\left(\mathrm{M}+\mathrm{Na}^{+}\right)$; 986.3400 , found 986.3399 .

To a stirred solution of [2-[3-bromo-4-(tert-butyldiphenylsilyloxy)phenyl]-1-(tertbutyldiphenylsilyloxymethyl)ethyl]tritylamine $(79.8 \mathrm{mg}, 0.083 \mathrm{mmol})$ in THF $(1.0 \mathrm{~mL})$ was added TBAF $(0.08 \mathrm{~mL}(1.0 \mathrm{M}$ solution in $\mathrm{THF}), 0.08 \mathrm{mmol})$ at $0^{\circ} \mathrm{C}$ under a nitrogen atmosphere. The reaction mixture was stirred for $0.5 \mathrm{~h}$ at $0^{\circ} \mathrm{C}$. The mixture was then quenched with aqueous saturated $\mathrm{NH}_{4} \mathrm{Cl}$ and extracted with AcOEt. The combined organics were washed with brine, dried, and concentrated in vacuo. The residue was purified by flash chromatography ( $n$-hexane/AcOEt=10/1) to give [2-[3-bromo-4- 
hydroxyphenyl]-1-(tert-butyldiphenylsilyloxymethyl)ethyl]tritylamine (47.1 $\mathrm{mg}, 78 \%)$ as a colorless oil.

IR (KBr): v 2930, 2862, 1493, 1470, 1448, $1427 \mathrm{~cm}^{-1} .{ }^{1} \mathrm{H}$ NMR (300 MHz): $\delta 1.09$ (s, 9H), $2.28(\mathrm{dd}, 1 \mathrm{H}, J=13.0,4.0 \mathrm{~Hz}), 2.40(\mathrm{dd}, 1 \mathrm{H}, J=13.0,9.0 \mathrm{~Hz}), 2.73(\mathrm{~m}, 1 \mathrm{H}), 3.16$ $(\mathrm{dd}, 1 \mathrm{H}, J=10.0,5.0 \mathrm{~Hz}), 3.30(\mathrm{dd}, 1 \mathrm{H}, J=10.0,3.5 \mathrm{~Hz}), 5.34(\mathrm{br} \mathrm{s}, 1 \mathrm{H}), 6.68(\mathrm{dd}, 1 \mathrm{H}$, $J=8.0,2.0 \mathrm{~Hz}), 6.77(\mathrm{~d}, 1 \mathrm{H}, J=8.5 \mathrm{~Hz}), 7.04(\mathrm{~d}, 1 \mathrm{H}, J=1.5 \mathrm{~Hz}), 7.15-7.45(\mathrm{~m}, 15 \mathrm{H})$, $7.52(\mathrm{~d}, 6 \mathrm{H}, J=7.0 \mathrm{~Hz}), 7.60(\mathrm{~d}, 2 \mathrm{H}, J=6.5 \mathrm{~Hz}), 7.67(\mathrm{~d}, 2 \mathrm{H}, J=6.5 \mathrm{~Hz}) .{ }^{13} \mathrm{C} \mathrm{NMR}$ (75 MHz): $\delta 19.3,27.0,36.8,55.4,64.4,71.1,109.7,115.4,126.3,127.6,127.8,128.7$, 129.6, 129.6, 130.2, 132.7, 133.1, 133.5, 133.6, 135.0, 135.7, 146.9, 150.2. HR-FABMS calcd for $\mathrm{C}_{44} \mathrm{H}_{44} \mathrm{BrNO}_{2} \mathrm{SiNa}\left(\mathrm{M}+\mathrm{Na}^{+}\right) ; 748.2223$, found 748.2206.

$0.1 \mathrm{~N} \mathrm{MeOH}$ solution of $\mathrm{HCl}(3.0 \mathrm{~mL})$ was added to [2-[3-bromo-4-hydroxyphenyl]1-(tert-butyldiphenylsilyloxymethyl)ethyl]tritylamine $(146.0 \mathrm{mg}, 0.201 \mathrm{mmol})$ at room temperature under a nitrogen atmosphere and the mixture was stirred for $30 \mathrm{~min}$. The above mixture was added dropwise to a stirred solution of $\mathbf{1 6 b}(71.6 \mathrm{mg}, 0.201 \mathrm{mmol})$ in $\mathrm{MeOH}(3.0 \mathrm{~mL})$ at room temperature under a nitrogen atmosphere. The mixture was stirred for $24 \mathrm{~h}$ under the same conditions and was then concentrated in vacuo. The residue was purified by flash chromatography $\left(\mathrm{CH}_{2} \mathrm{Cl}_{2} / \mathrm{MeOH} / \mathrm{Et}_{3} \mathrm{~N}=100: 5: 0.1\right)$ to give 20c (97.4 mg, 60\%) as a red amorphous solid.

20c: IR (KBr): v 2930, 2856, 1666, 1580, 1556, $1537 \mathrm{~cm}^{-1} .{ }^{1} \mathrm{H}$ NMR $(500 \mathrm{MHz}$, $\left.\mathrm{CD}_{3} \mathrm{OD}\right): \delta 1.04(\mathrm{~s}, 9 \mathrm{H}), 2.36(\mathrm{~s}, 3 \mathrm{H}), 2.78(\mathrm{t}, 2 \mathrm{H}, J=7.5 \mathrm{~Hz}), 2.83(\mathrm{dd}, 1 \mathrm{H}, J=13.5$, $6.5 \mathrm{~Hz}$ ), $2.90(\mathrm{dd}, 1 \mathrm{H}, J=13.5,7.5 \mathrm{~Hz}), 3.50-3.52(\mathrm{~m}, 2 \mathrm{H}), 3.66-3.68(\mathrm{~m} .1 \mathrm{H}), 3.94(\mathrm{t}$, $2 \mathrm{H}, J=7.5 \mathrm{~Hz}), 5.28$ (br s, 1H), $6.78(\mathrm{~d}, 1 \mathrm{H}, J=8.5 \mathrm{~Hz}), 6.98-7.02(\mathrm{~m}, 3 \mathrm{H}), 7.20$ (t, $1 \mathrm{H}, J=7.5 \mathrm{~Hz}), 7.27(\mathrm{t}, 1 \mathrm{H}, J=7.5 \mathrm{~Hz}), 7.33-7.38(\mathrm{~m}, 6 \mathrm{H}), 7.50(\mathrm{~d}, 2 \mathrm{H}, J=6.5 \mathrm{~Hz})$, 7.70 (br s, $1 \mathrm{H}), 8.08$ (d, 2H, $J=8.0 \mathrm{~Hz}) .{ }^{13} \mathrm{C}$ NMR (125 MHz, $\left.\mathrm{CD}_{3} \mathrm{OD}\right): \delta 18.9,19.9$, 21.8, 27.4, 36.0, 43.6, 59.0, 64.0, 87.1, 110.8, 117.4, 119.9, 124.2, 128.8, 128.9, 130.3, $130.6,130.7,130.9,131.0,131.3,133.8,133.9$, 134.6, 134.9, 136.6, 136.7, 148.7, 153.3, 154.3, 157.8, 166.8. HR-FABMS calcd for $\mathrm{C}_{42} \mathrm{H}_{43} \mathrm{BrN}_{3} \mathrm{O}_{5} \mathrm{SSi}\left(M+\mathrm{H}^{+}\right)$; 808.1876 , found 808.1848 . 


\section{5-(Toluene-4-sulfonyl)-3,5,7,8,9,10-hexahydro-2H-1,5,7-triaza-}

acephenanthrylen-6-one-8-(tert-butyldiphenylsilyloxymethyl)-10-spiro-4'-(2'bromo)-cyclohexa-2',5'-dien-1'-one (21c, 21'c).

To a stirred solution of $20 \mathrm{c}(33.8 \mathrm{mg}, 0.0418 \mathrm{mmol})$ in $\mathrm{CF}_{3} \mathrm{CH}_{2} \mathrm{OH}(1.5 \mathrm{~mL})$ was added MK10 (7.3 mg) and PIFA (21.6 mg, $0.0501 \mathrm{mmol})$ sequentially at room temperature. The mixture was stirred for $45 \mathrm{~min}$. under the same conditions and then concentrated in vacuo. The residue was purified by flash chromatography ( $n$ hexane/AcOEt/ $\left.\mathrm{Et}_{3} \mathrm{~N}=100: 50: 0.1\right)$ to afford two diastereomers 21c $(6.4 \mathrm{mg}, 19 \%)$ and 21 'c (4.3 mg, 13\%) respectively as a red amorphous solid.

21c (less polar): IR (KBr): $v$ 1661, 1528, 1483, 1460, $1379 \mathrm{~cm}^{-1} .{ }^{1} \mathrm{H}$ NMR (500 MHz): $\delta 1.08(\mathrm{~s}, 9 \mathrm{H}), 1.51(\mathrm{~d}, 1 \mathrm{H}, J=13.0 \mathrm{~Hz}), 1.68(\mathrm{t}, 1 \mathrm{H}, J=11.5 \mathrm{~Hz}), 2.41(\mathrm{~s}, 3 \mathrm{H})$, $2.60(\mathrm{t}, 2 \mathrm{H}, J=7.5 \mathrm{~Hz}), 3.55(\mathrm{~m}, 2 \mathrm{H}), 3.71(\mathrm{dd}, 1 \mathrm{H}, J=10.0,3.5 \mathrm{~Hz}), 3.82(\mathrm{dt}, 1 \mathrm{H}, J=$ $17.5,8.5 \mathrm{~Hz}), 4.07$ (dt, 1H, $J=18.0,7.0 \mathrm{~Hz}), 6.12$ (br s, 1H), 6.25 (d, 1H, $J=10.0 \mathrm{~Hz}$ ), $6.89(\mathrm{dd}, 1 \mathrm{H}, J=9.5,2.5 \mathrm{~Hz}), 7.22(\mathrm{~d}, 1 \mathrm{H}, J=2.0 \mathrm{~Hz}), 7.32(\mathrm{~d}, 2 \mathrm{H}, J=8.0 \mathrm{~Hz}), 7.37-$ $7.44(\mathrm{~m}, 7 \mathrm{H}), 7.63$ (d, 4H, $J=7.5 \mathrm{~Hz}), 8.04$ (d, 2H, $J=8.0 \mathrm{~Hz}) .{ }^{13} \mathrm{C}$ NMR $(75 \mathrm{MHz}): \delta$ $17.5,19.2,21.7,26.9,36.5,43.8,49.4,49.7,66.7,104.1,118.5,121.8,121.9,125.4$, $125.8,126.1,127.9,128.7,129.7,130.0,132.6,132.7,134.6,135.5,141.9,145.7,152.2$, 152.7, 157.1, 168.4, 179.0. UV/Vis $\left(\mathrm{CHCl}_{3}\right): \lambda \max (\varepsilon)=485$ (1900), 330 (11100), 230 nm (33100). HR-FABMS calcd for $\mathrm{C}_{42} \mathrm{H}_{43} \mathrm{BrN}_{3} \mathrm{O}_{5} \mathrm{SSi}\left(M+\mathrm{H}^{+}\right) ; 806.1720$, found 806.1780 .

21'c (polar): IR (KBr): v 1661, 1526, 1487, 1462, $1379 \mathrm{~cm}^{-1} .{ }^{1} \mathrm{H}$ NMR (500 MHz): $\delta$ $1.11(\mathrm{~s}, 9 \mathrm{H}), 1.50(\mathrm{~d}, 1 \mathrm{H}, J=14.0 \mathrm{~Hz}), 1.69$ (t, 1H, $J=11.5 \mathrm{~Hz}), 2.43$ (s, 3H), 2.61 (t, $2 \mathrm{H}, J=7.0 \mathrm{~Hz}), 3.53-3.59(\mathrm{~m}, 2 \mathrm{H}), 3.71-3.86(\mathrm{~m}, 2 \mathrm{H}), 4.06(\mathrm{dt}, 1 \mathrm{H}, J=18.0,6.5 \mathrm{~Hz})$, 6.19 (br s, 1H), $6.31(\mathrm{~d}, 1 \mathrm{H}, J=9.5 \mathrm{~Hz}), 6.86(\mathrm{dd}, 1 \mathrm{H}, J=9.5,2.5 \mathrm{~Hz}), 7.33(\mathrm{~d}, 2 \mathrm{H}, J=$ $7.5 \mathrm{~Hz}), 7.39-7.47(\mathrm{~m}, 8 \mathrm{H}), 7.66(\mathrm{~d}, 4 \mathrm{H}, J=7.0 \mathrm{~Hz}), 8.06(\mathrm{~d}, 2 \mathrm{H}, J=8.5 \mathrm{~Hz}) .{ }^{13} \mathrm{C} \mathrm{NMR}$ (75 MHz): $\delta 17.6,19.2,21.8,26.9,36.7,44.0,49.4,49.5,66.7,104.1,118.5,121.9$, $123.8,124.9,125.5,126.0,127.9,128.8,129.7,130.0,130.1,132.6,132.7,134.6,135.5$, $135.6,142.0,145.8,152.2,152.8,157.0,168.3,179.0 . \mathrm{UV} / \mathrm{Vis}\left(\mathrm{CHCl}_{3}\right): \lambda \max (\varepsilon)=483$ 
(2100), 330 (12400), $230 \mathrm{~nm}$ (34600). HR-FABMS calcd for $\mathrm{C}_{42} \mathrm{H}_{43} \mathrm{BrN}_{3} \mathrm{O}_{5} \mathrm{SSi}$ $\left(M+\mathrm{H}^{+}\right) ; 806.1719$, found 806.1711.

\section{5-(Toluene-4-sulfonyl)-3,5,7,8,9,10-hexahydro-2H-1,5,7-triaza-}

acephenanthrylen-6-one-8-(tert-butyldimethylsilyloxymethyl)-10-spiro-4'-(2'bromo)-cyclohexa-2',5'-dien-1'-one (21a, 21'a).

To a stirred solution of 20a $(54.1 \mathrm{mg}, 0.079 \mathrm{mmol})$ in anhydrous $\mathrm{CH}_{2} \mathrm{Cl}_{2}(1.6 \mathrm{~mL})$ was added 1-methoxy-2-methyl-1-(trimethylsiloxy)propene $(0.21 \mathrm{~mL}, 1.19 \mathrm{mmol})$ at room temperature under a nitrogen atmosphere. The mixture was stirred for $7 \mathrm{~h}$ under the same conditions and then concentrated in vacuo to give 20d. To a stirred solution of the residue (crude 20d) in $\mathrm{CF}_{3} \mathrm{CH}_{2} \mathrm{OH}(1.3 \mathrm{~mL})$ was added MK10 $(16.6 \mathrm{mg})$ and PIFA $(67.9 \mathrm{mg}, 0.158 \mathrm{mmol})$ sequentially at room temperature. The mixture was stirred for 1 $\mathrm{h}$ under the same conditions and then concentrated in vacuo. The residue was purified by flash chromatography ( $n$-hexane/AcOEt/Et $3 \mathrm{~N}=100: 50: 0.1)$ to afford two diastereomers $\mathbf{2 1 a}(5.2 \mathrm{mg}, 12.8 \%)$ and $\mathbf{2 1}$ 'a $(2.9 \mathrm{mg}, 7.1 \%)$ respectively as a red amorphous solid respectively.

\section{7-[2-(3-Bromo-4-triisopropylsilyloxyphenyl)-1-(tert-butyldimethylsilyloxy-} methyl)ethylamino]-1-(toluene-4-sulfonyl)-3,4-dihydro-1 $H$-pyrrolo[4,3,2-de]quinolin-8-one (20f).

To a stirred solution of 20a $(41.3 \mathrm{mg}, 0.0603 \mathrm{mmol})$ in DMF $(2.0 \mathrm{~mL})$ was added imidazole (6.2 mL, $0.0905 \mathrm{mmol})$ and TIPSCl (0.064 mL, $0.302 \mathrm{mmol})$ sequentially at $0^{\circ} \mathrm{C}$ under a nitrogen atmosphere. The reaction mixture was stirred for $3 \mathrm{~h}$ at $0^{\circ} \mathrm{C}$. The mixture was then quenched with aqueous saturated $\mathrm{NaHCO}_{3}$ and extracted with AcOEt. The combined organics were washed with brine, dried, and concentrated in vacuo. The residue was purified by flash chromatography ( $n$-hexane/AcOEt/Et ${ }_{3} \mathrm{~N}=100 / 100 / 0.1$ ) to give $20 f(33.0 \mathrm{mg}, 65 \%)$ as a red oil.

20f: IR (KBr): v 2947, 2928, 2866, 1661, 1580, 1531, $1493 \mathrm{~cm}^{-1} .{ }^{1} \mathrm{H}$ NMR (500 MHz, $\left.\mathrm{CD}_{3} \mathrm{OD}\right): \delta 0.02(\mathrm{~s}, 6 \mathrm{H}), 0.89(\mathrm{~s}, 9 \mathrm{H}), 1.00-1.32(\mathrm{~m}, 21 \mathrm{H}), 2.42(\mathrm{~s}, 3 \mathrm{H}), 2.73-2.77(\mathrm{~m}$, $3 \mathrm{H}), 2.83(\mathrm{dd}, 1 \mathrm{H}, J=14.0,6.5 \mathrm{~Hz}), 3.54-3.68(\mathrm{~m}, 3 \mathrm{H}), 3.96(\mathrm{t}, 2 \mathrm{H}, J=6.5 \mathrm{~Hz}), 5.48$ (br s, 1H), $6.78(\mathrm{~d}, 1 \mathrm{H}, J=8.5 \mathrm{~Hz}), 7.04(\mathrm{dd}, 1 \mathrm{H}, J=8.0,2.0 \mathrm{~Hz}), 7.37(\mathrm{~d}, 1 \mathrm{H}, J=2.0$ 
Hz), 7.39 (d, 2H, $J=8.5 \mathrm{~Hz}), 7.65$ (br s, 1H), 8.02 (d, 2H, $J=8.5 \mathrm{~Hz}$ ). HR-FABMS calcd for $\mathrm{C}_{41} \mathrm{H}_{59} \mathrm{BrN}_{3} \mathrm{O}_{5} \mathrm{SSi}_{2}\left(M+\mathrm{H}^{+}\right) ;$840.2897, found 840.2904. This compound gradually decomposed during ${ }^{13} \mathrm{C}$ NMR measurement due to its unstability.

\section{5-(Toluene-4-sulfonyl)-3,5,7,8,9,10-hexahydro-2H-1,5,7-triaza-}

acephenanthrylen-6-one-8-(tert-butyldimethylsilyloxymethyl)-10-spiro-4'-(2'bromo)-cyclohexa-2',5'-dien-1'-one (21a, 21'a).

To a stirred solution of $20 f(18.0 \mathrm{mg}, 0.0213 \mathrm{mmol})$ in $\mathrm{CF}_{3} \mathrm{CH}_{2} \mathrm{OH}(0.7 \mathrm{~mL})$ was added MK10 (12.4 mg) and PIFA (36.6 mg, $0.0852 \mathrm{mmol})$ sequentially at room temperature. The mixture was stirred for $7 \mathrm{~h}$. under the same conditions and then concentrated in vacuo. The residue was purified by flash chromatography ( $n$ hexane/AcOEt $\left./ \mathrm{Et}_{3} \mathrm{~N}=100: 50: 0.1\right)$ to afford two diastereomers 21a (4.2 $\left.\mathrm{mg}, 28.8 \%\right)$ and 21'a (1.1 mg, 7.2\%) respectively as a red amorphous solid.

\section{7-[2-(3-Bromo-4-tert-butyldiphenylsilyloxyphenyl)-1-(tert-butyldimethylsilyl-} oxymethyl)ethylamino]-1-(toluene-4-sulfonyl)-3,4-dihydro-1H-pyrrolo[4,3,2-de]quinonlin-8-one (20g).

To a stirred solution of $\mathbf{2 0 a}(43.3 \mathrm{mg}, 0.0632 \mathrm{mmol})$ in THF $(2.0 \mathrm{~mL})$ was added imidazole (12.9 mg, $0.190 \mathrm{mmol})$ and TBDPSCl (0.049 mL, $0.190 \mathrm{mmol})$ sequentially at $0^{\circ} \mathrm{C}$ under a nitrogen atmosphere. The reaction mixture was stirred for $4 \mathrm{~h}$ at $0^{\circ} \mathrm{C}$. The mixture was then quenched with aqueous saturated $\mathrm{NaHCO}_{3}$ and extracted with AcOEt. The combined organics were washed with brine, dried, and concentrated in vacuo. The residue was purified by flash chromatography ( $n$-hexane/AcOEt/Et $\left.{ }_{3} \mathrm{~N}=100 / 100 / 0.1\right)$ to give $20 \mathrm{~g}(22.5 \mathrm{mg}, 39 \%)$ as a red oil.

20g: IR (KBr): v 2947, 2928, 2866, 1661, 1580, 1531, $1493 \mathrm{~cm}^{-1} .{ }^{1} \mathrm{H}$ NMR (500 MHz, $\left.\mathrm{CD}_{3} \mathrm{OD}\right): \delta-0.02(\mathrm{~s}, 6 \mathrm{H}), 0.87(\mathrm{~s}, 9 \mathrm{H}), 1.08(\mathrm{~s}, 9 \mathrm{H}), 2.37(\mathrm{~s}, 3 \mathrm{H}), 2.70-2.79(\mathrm{~m}, 4 \mathrm{H})$, 3.50-3.52 (m, 2H), $3.61(\mathrm{dd}, 1 \mathrm{H}, J=11.5,5.5 \mathrm{~Hz}), 3.97$ (t, 2H, $J=7.5 \mathrm{~Hz}), 5.46$ (br s, $1 \mathrm{H}), 6.34(\mathrm{~d}, 1 \mathrm{H}, J=8.0 \mathrm{~Hz}), 6.71(\mathrm{dd}, 1 \mathrm{H}, J=8.0,2.0 \mathrm{~Hz}), 7.32-7.36(\mathrm{~m}, 5 \mathrm{H}), 7.39(\mathrm{~d}$, $4 \mathrm{H}, J=7.0 \mathrm{~Hz}), 7.68$ (br s, 1H), 7.68 (d, 4H, $J=7.0 \mathrm{~Hz}), 7.99$ (d, 2H, $J=8.0 \mathrm{~Hz})$. HRFABMS calcd for $\mathrm{C}_{48} \mathrm{H}_{57} \mathrm{BrN}_{3} \mathrm{O}_{5} \mathrm{SSi}_{2}\left(M+\mathrm{H}^{+}\right)$; 922.2741, found 922.2740. This 
compound gradually decomposed during ${ }^{13} \mathrm{C}$ NMR measurement due to its unstability.

\section{5-(Toluene-4-sulfonyl)-3,5,7,8,9,10-hexahydro-2 $\mathrm{H}$-1,5,7-triaza-}

acephenanthrylen-6-one-8-(tert-butyldimethylsilyloxymethyl)-10-spiro-4'-(2'bromo)-cyclohexa-2',5'-dien-1'-one (21a, 21'a).

To a stirred solution of $20 \mathrm{~g}(7.8 \mathrm{mg}, 0.0084 \mathrm{mmol})$ in $\mathrm{CF}_{3} \mathrm{CH}_{2} \mathrm{OH}(0.5 \mathrm{~mL})$ was added MK10 (6.2 mg) and PIFA (18.2 mg, $0.0422 \mathrm{mmol})$ sequentially at room temperature. The mixture was stirred for $24 \mathrm{~h}$. under the same conditions and then concentrated in vacuo. The residue was purified by flash chromatography ( $n$ hexane/AcOEt/ $\left.\mathrm{Et}_{3} \mathrm{~N}=100: 50: 0.1\right)$ to afford two diastereomers $21 \mathrm{a}(2.1 \mathrm{mg}, 37.0 \%)$ and 21 'a $(0.7 \mathrm{mg}, 12.0 \%)$ respectively as a red amorphous solid. 
年

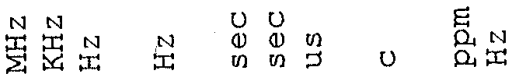

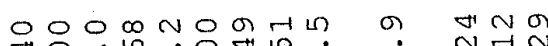

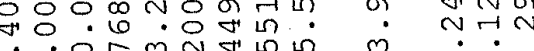

O유 $\mathrm{N} r \dot{0}$

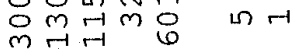

㞼管

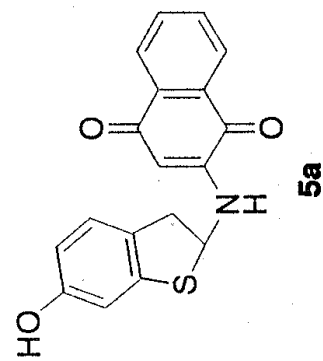

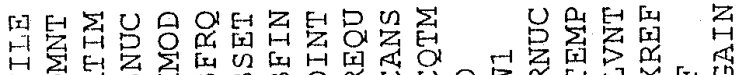

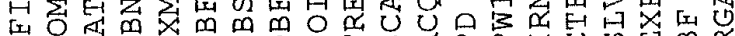

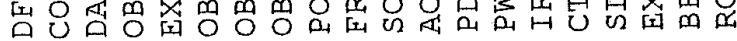

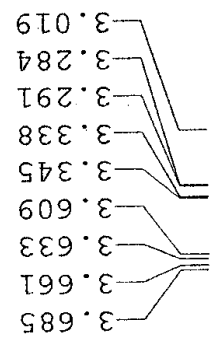

$s \emptyset \varepsilon \cdot s$

$\sigma L \varepsilon \cdot s-$

$86 \varepsilon^{\circ} \cdot \mathrm{s}$

$\varepsilon \varepsilon 8 \cdot 97$

$\varepsilon 8 z \cdot 9]$

$\varepsilon I \varepsilon \cdot 9-$
$09 G \cdot 9$

$985 \cdot 9]$

$80 L \cdot 9$

TOT $L \square$

$6 Z T \cdot L \square$

Oठ $2 \cdot L \neg=$

$\left.98 G^{\circ} L\right]$

$\left.\begin{array}{l}809^{\circ} L \\ L E 9^{\circ} L\end{array}\right]$

$L 9^{\circ} L$
$Z Z L$

$\sigma B L \cdot L=$

$L 66^{\circ} L \longrightarrow$

$\varepsilon 乙 0^{\circ}$

ZLO

$660 \cdot 8$

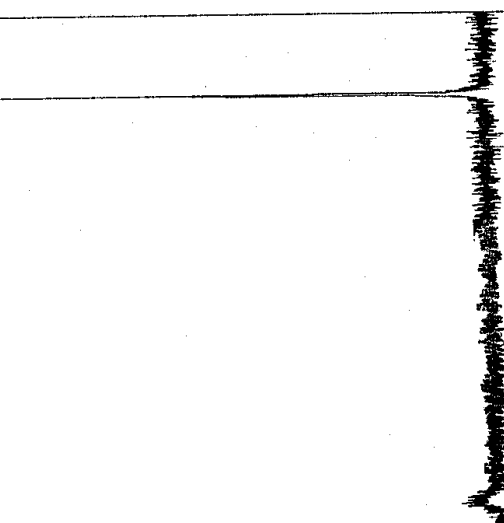

部 


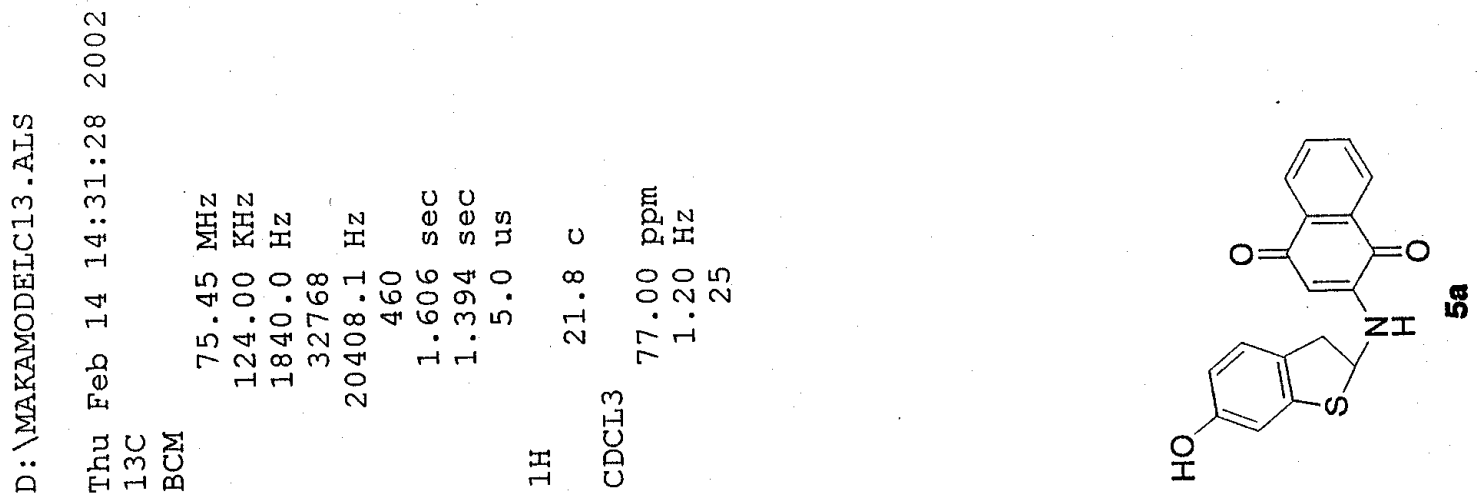

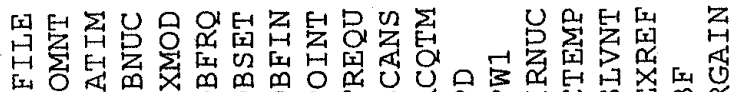

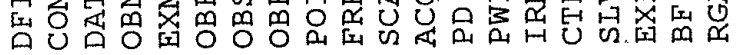

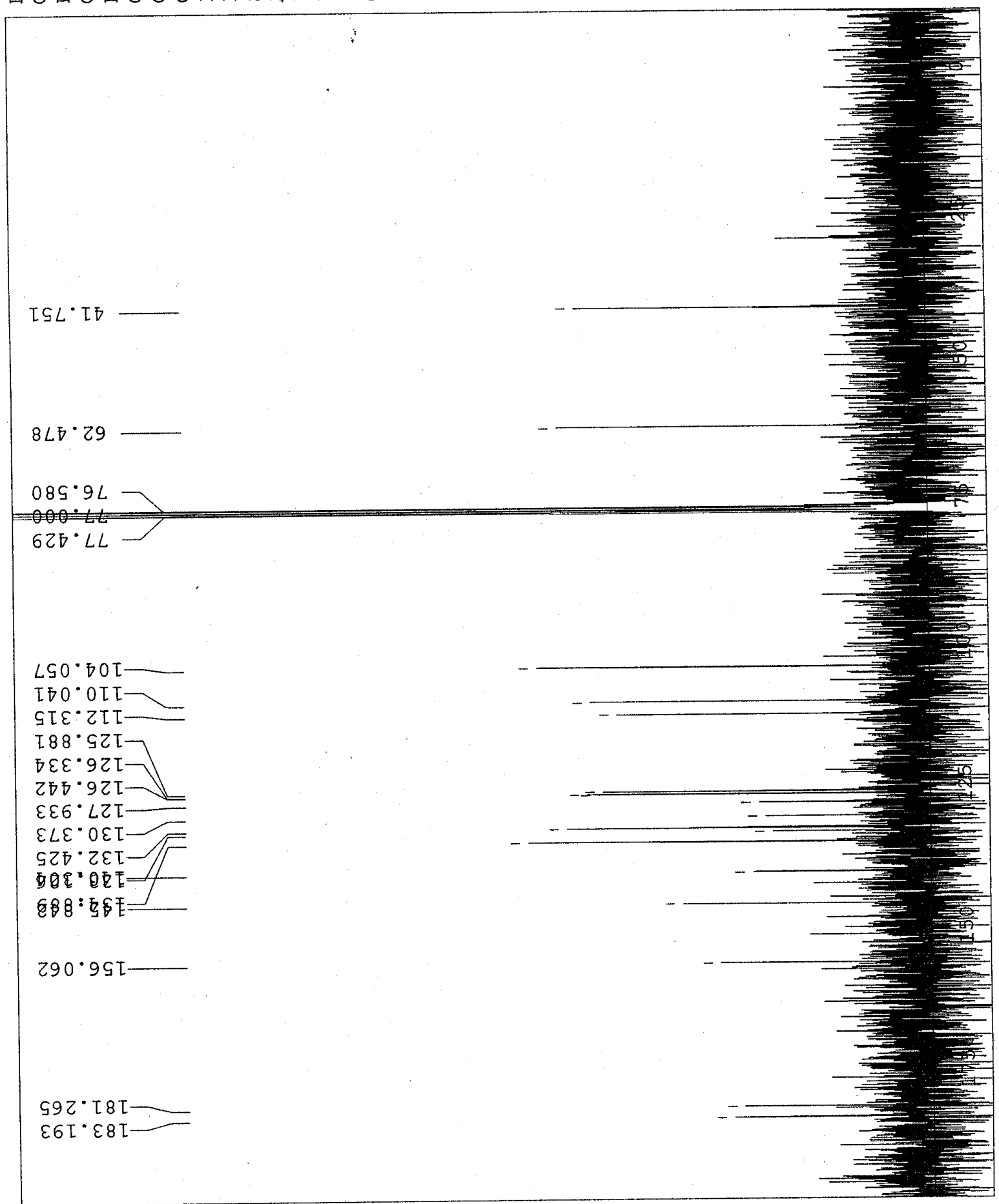




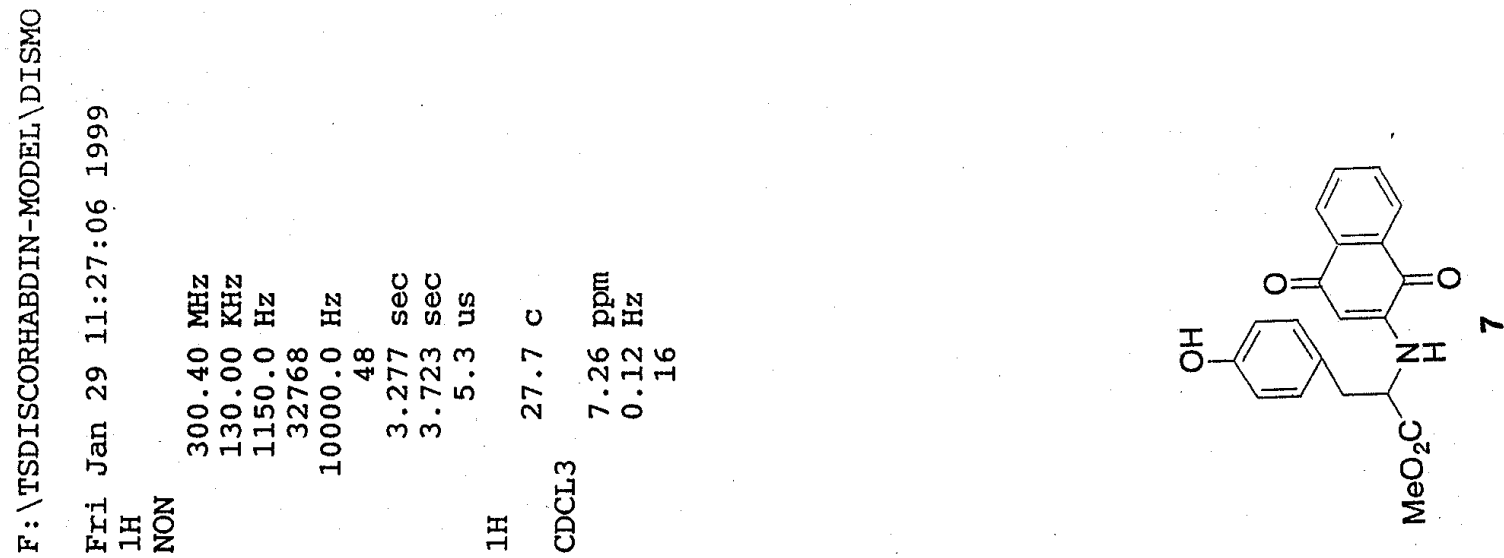

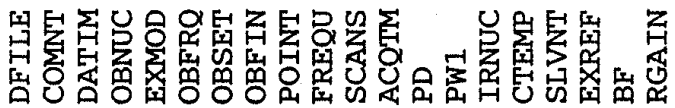

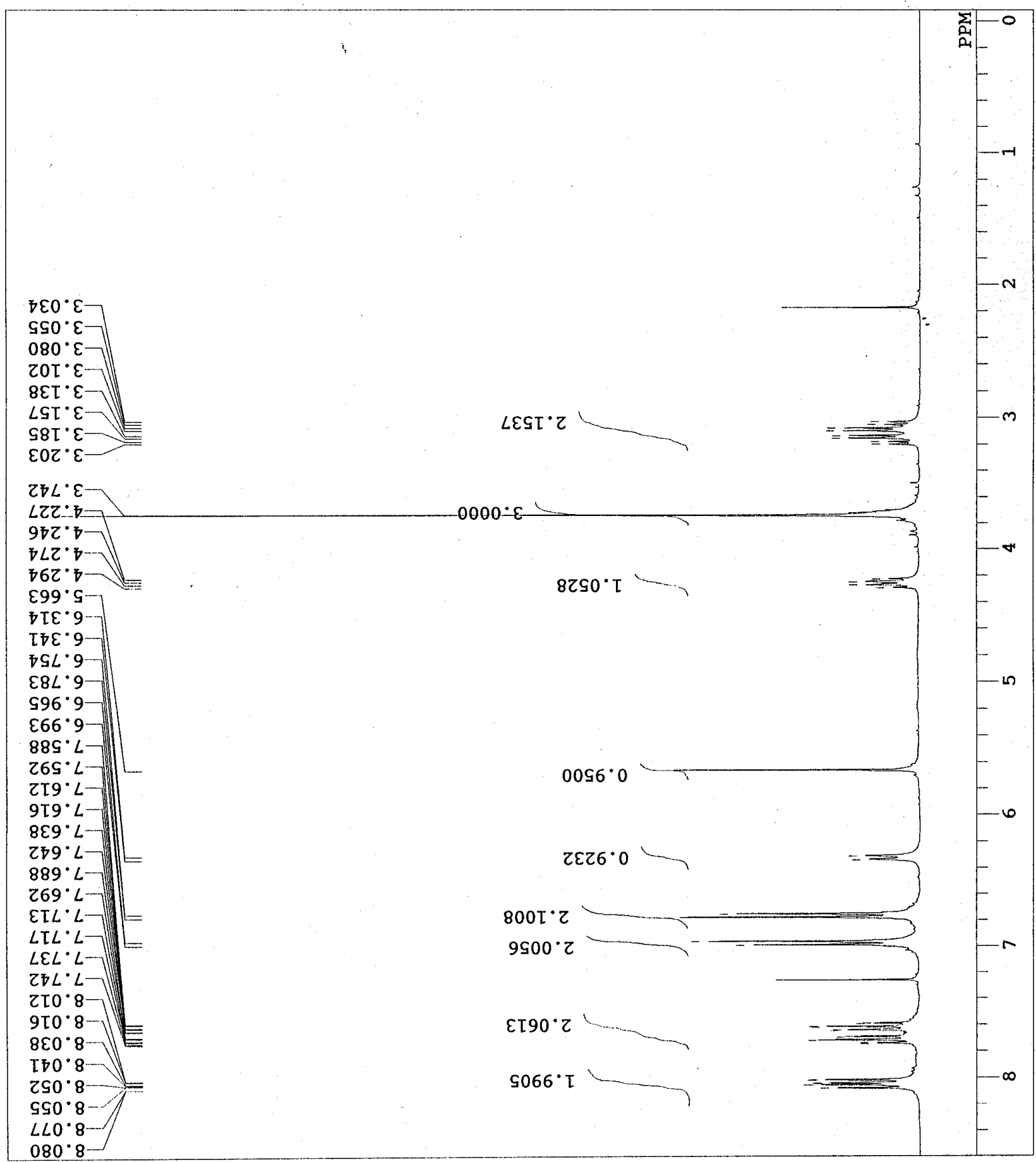




$$
1
$$




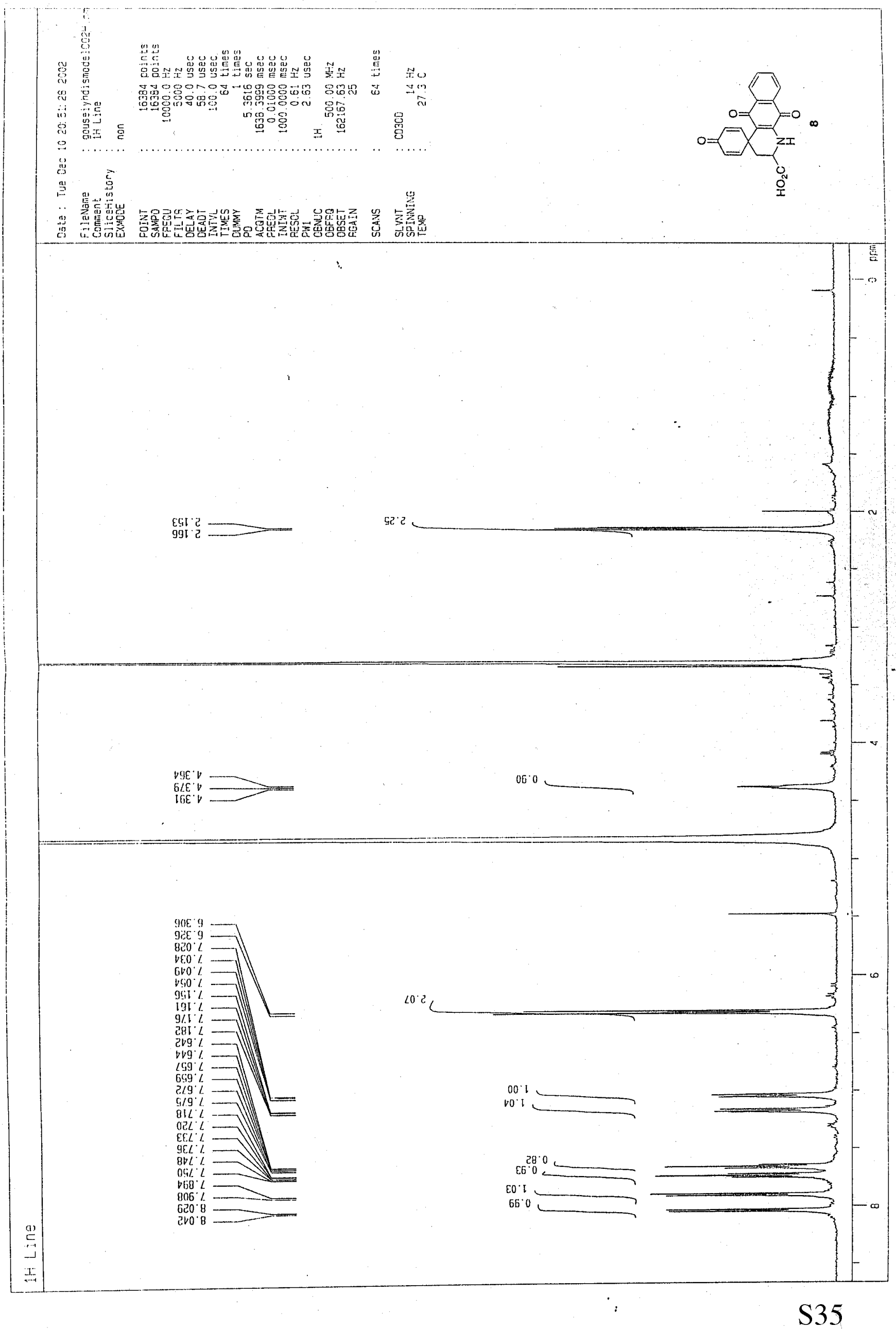




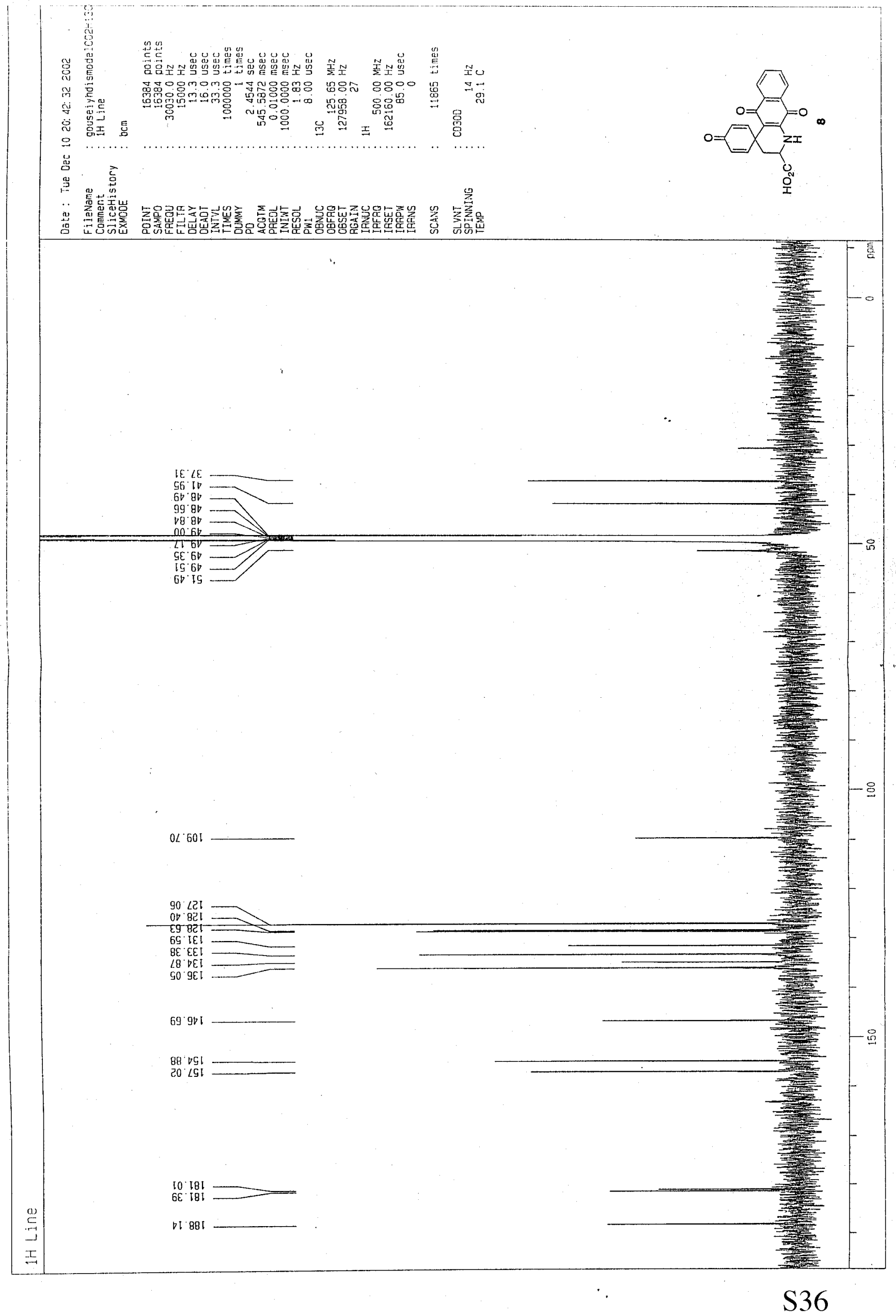




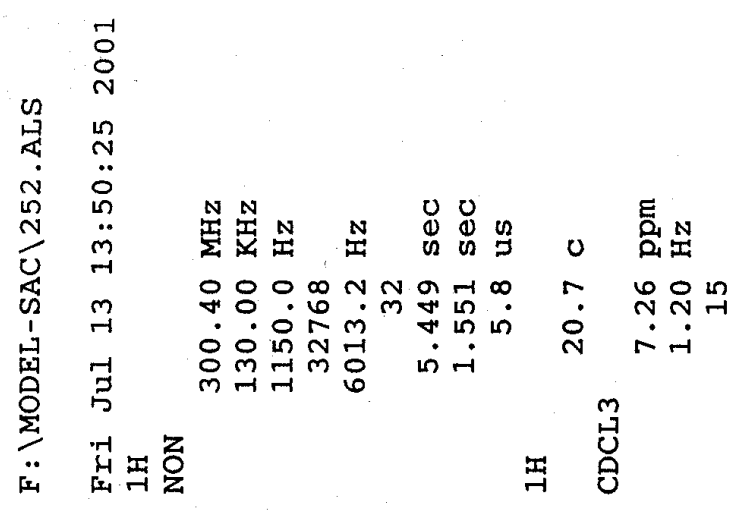

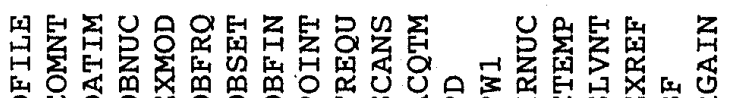

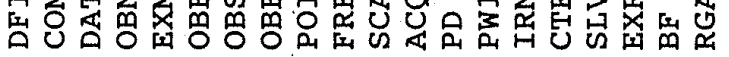

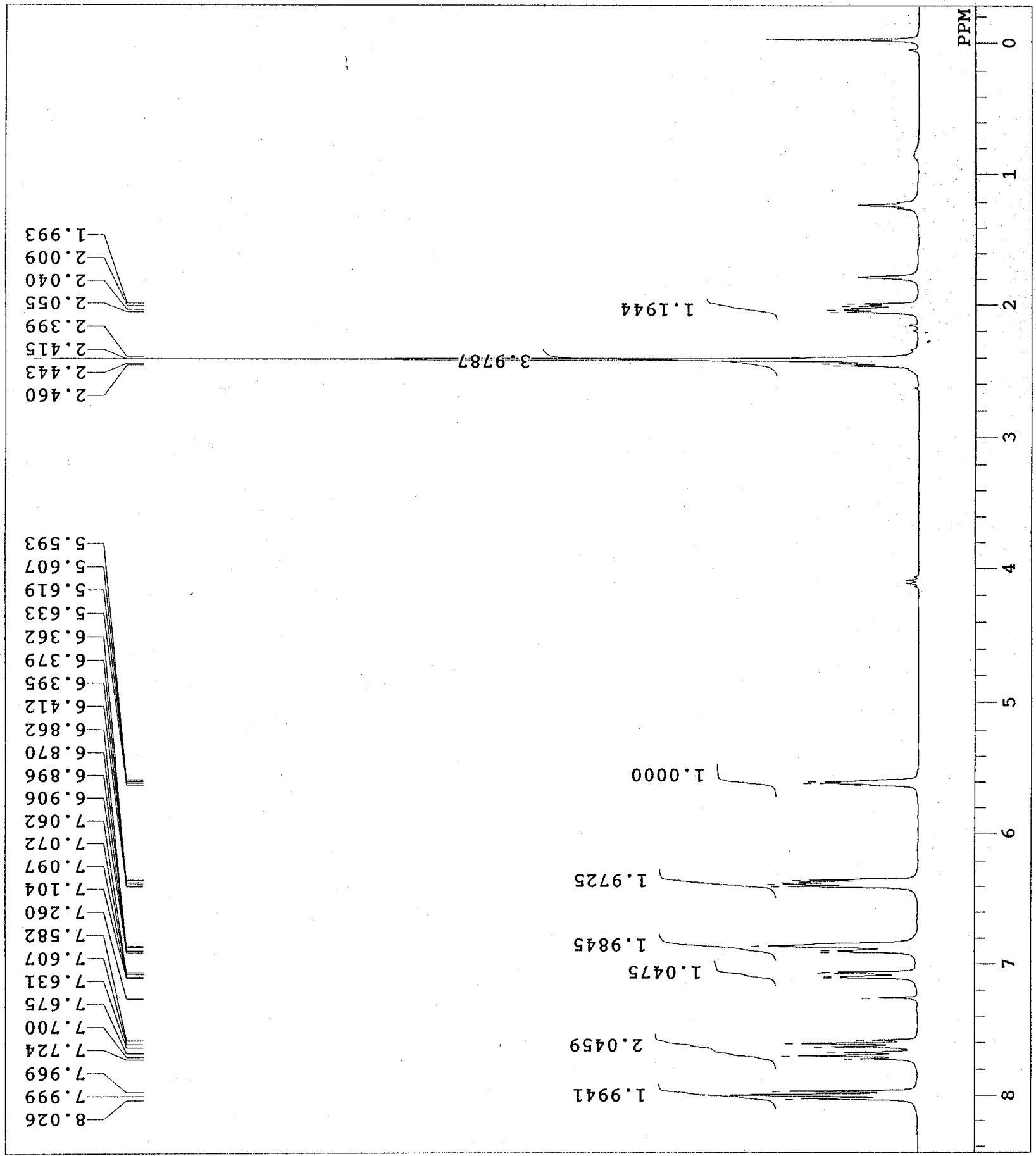




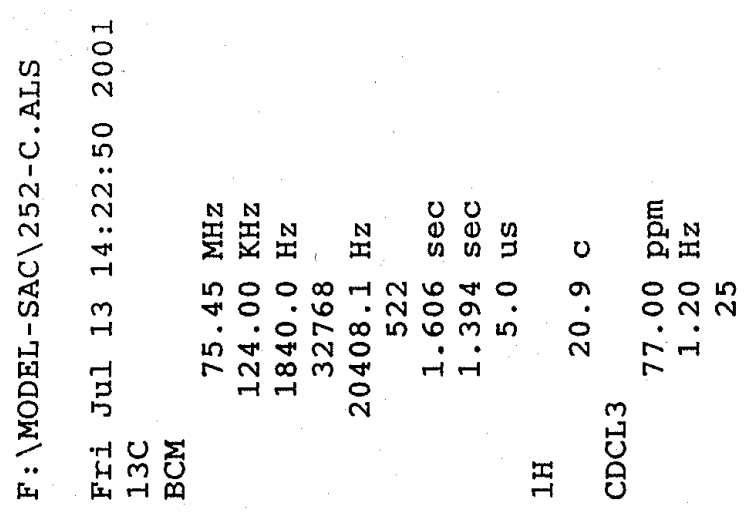

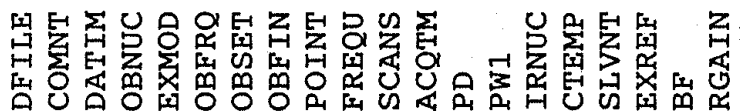
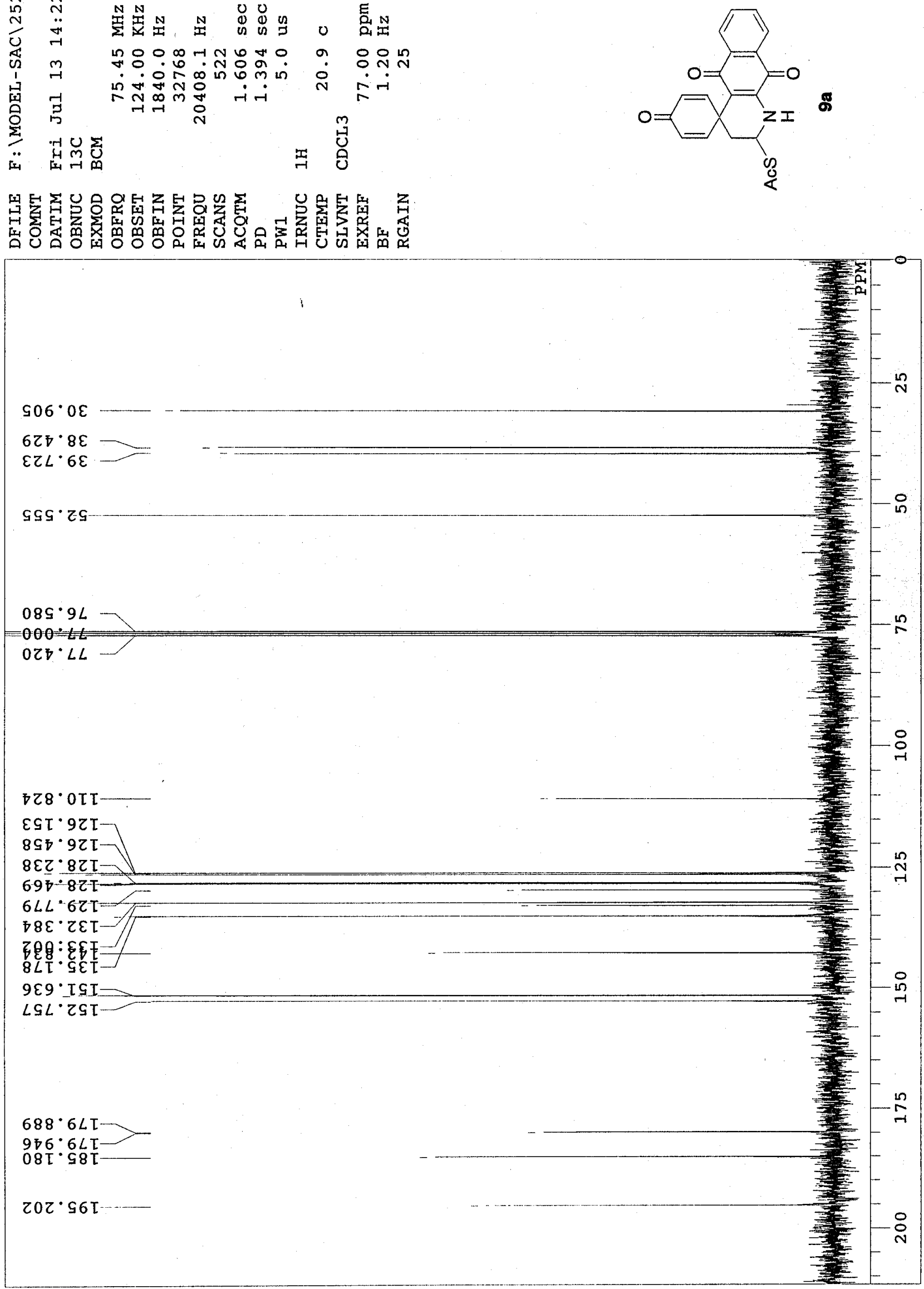


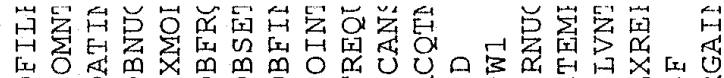

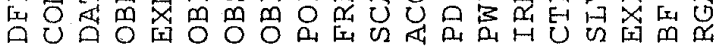

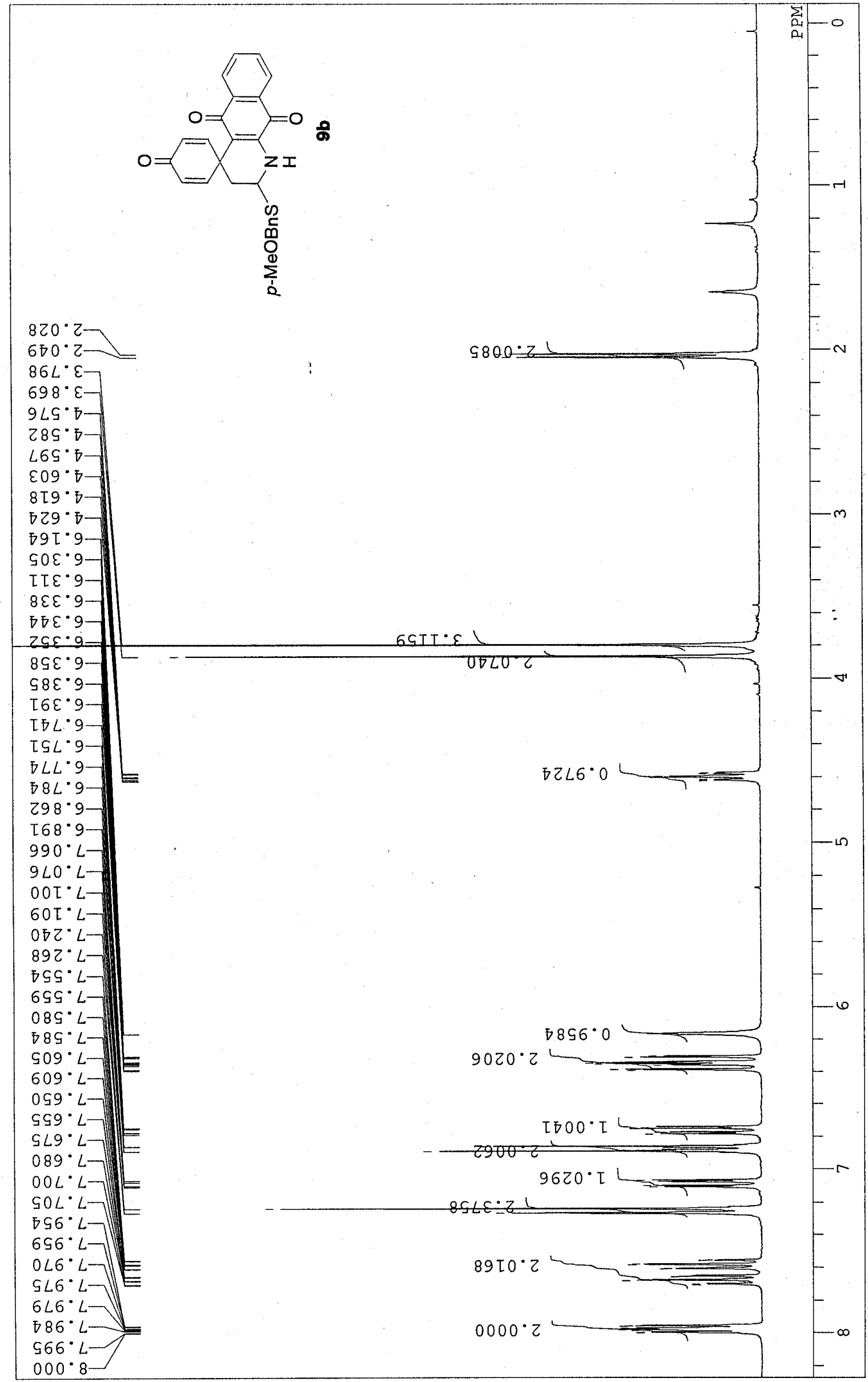




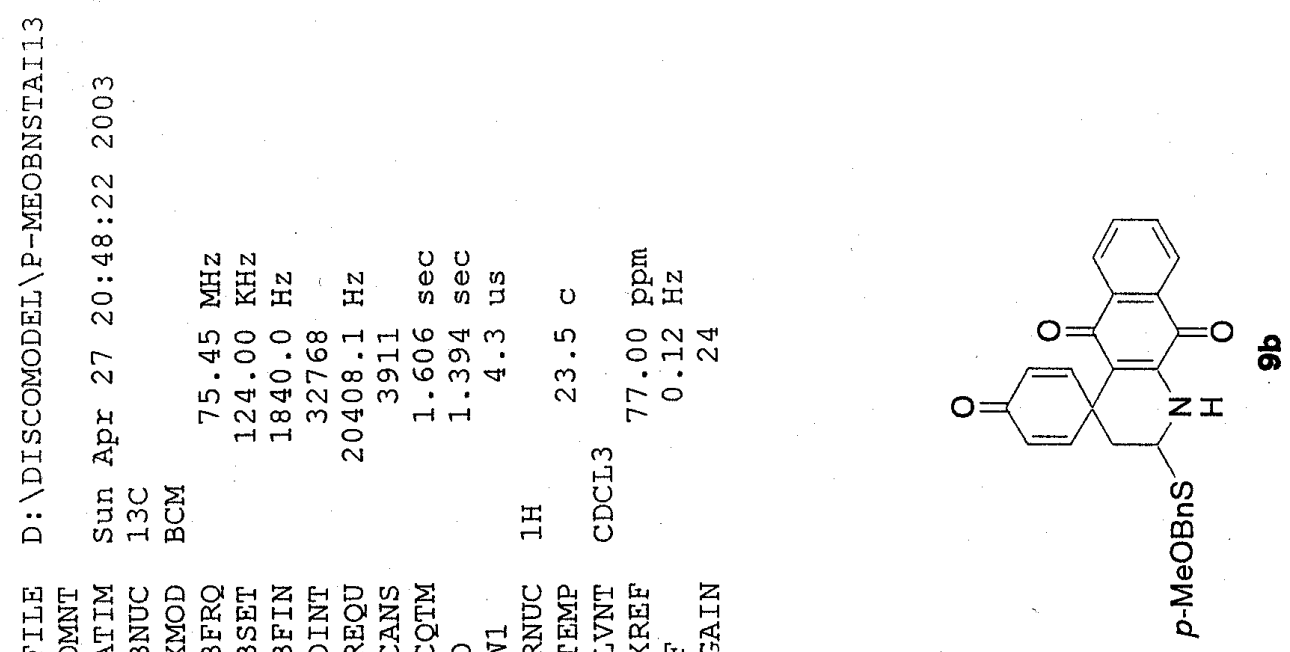

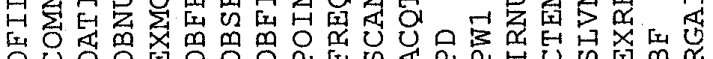

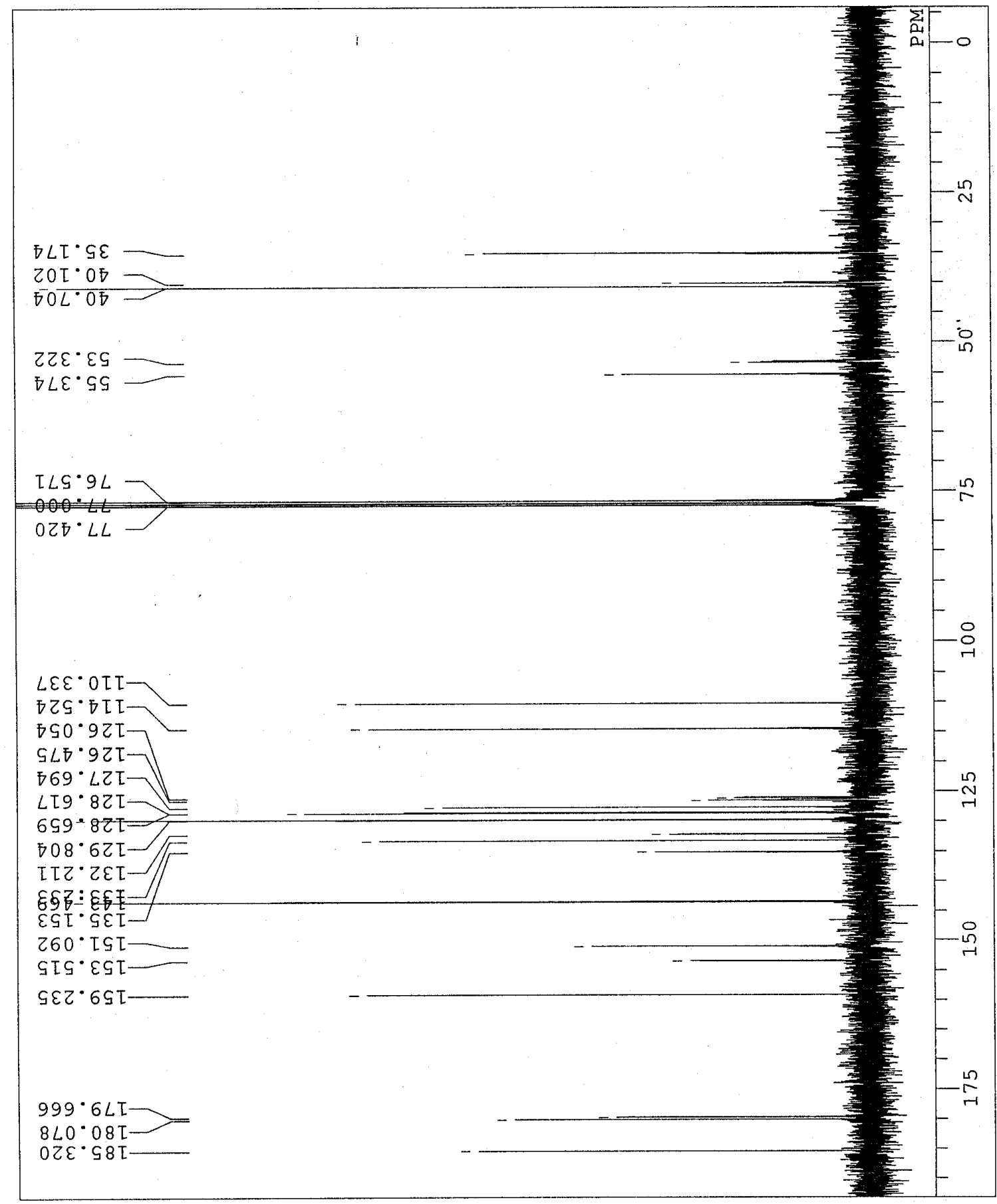




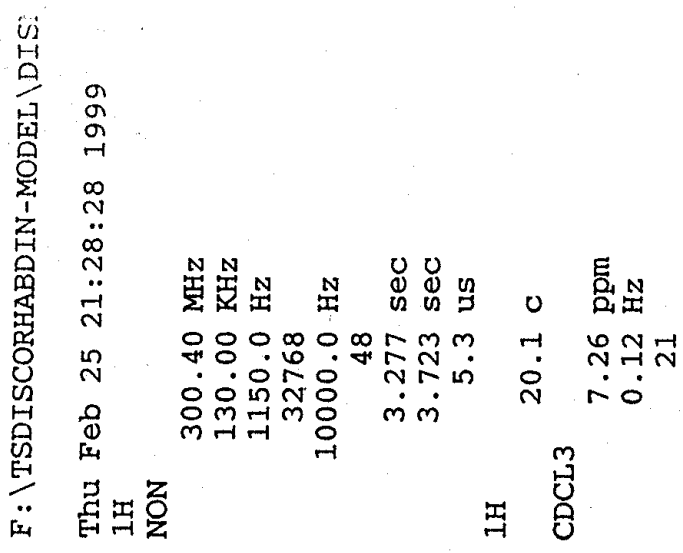

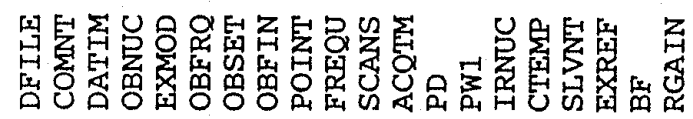
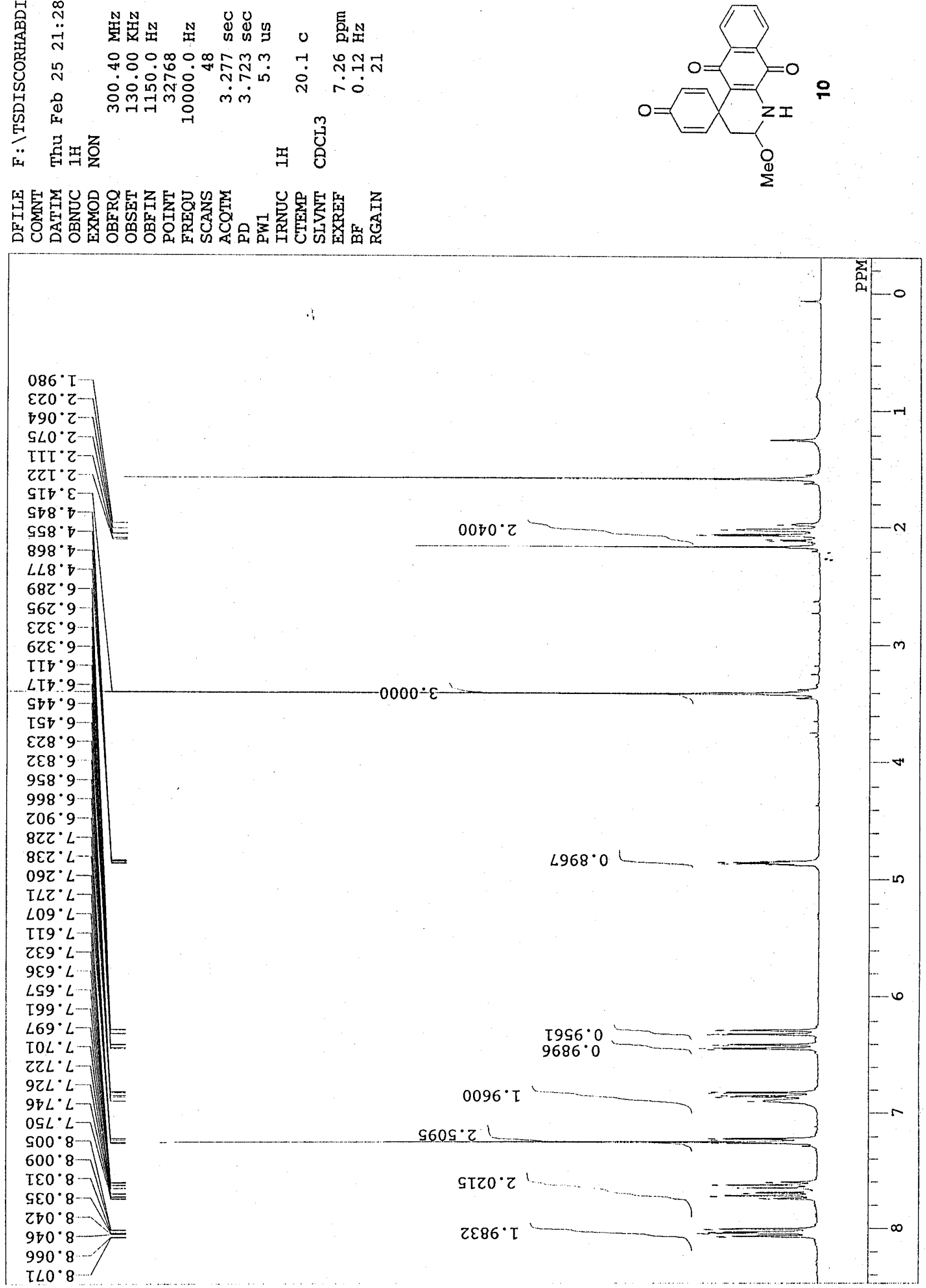


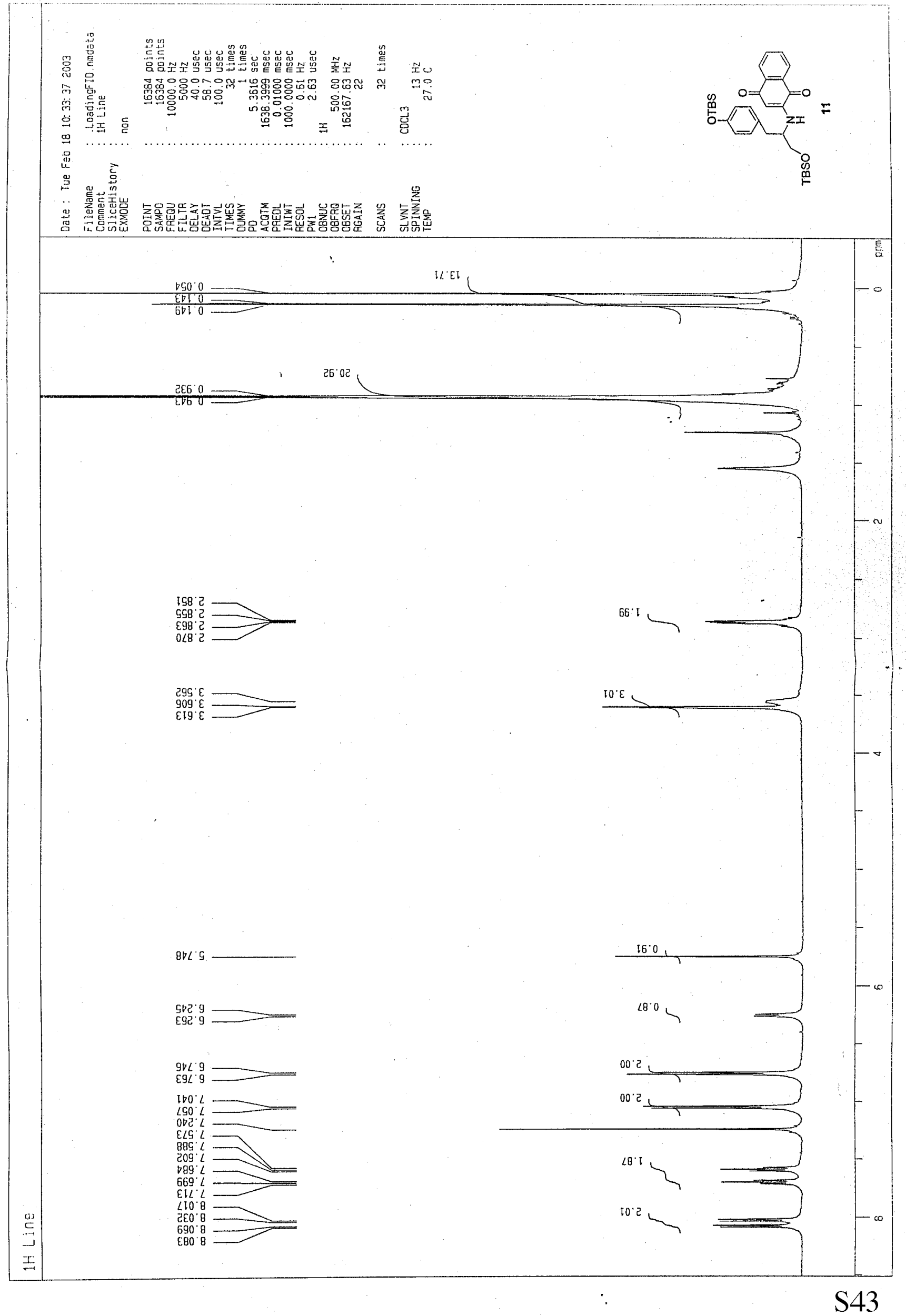




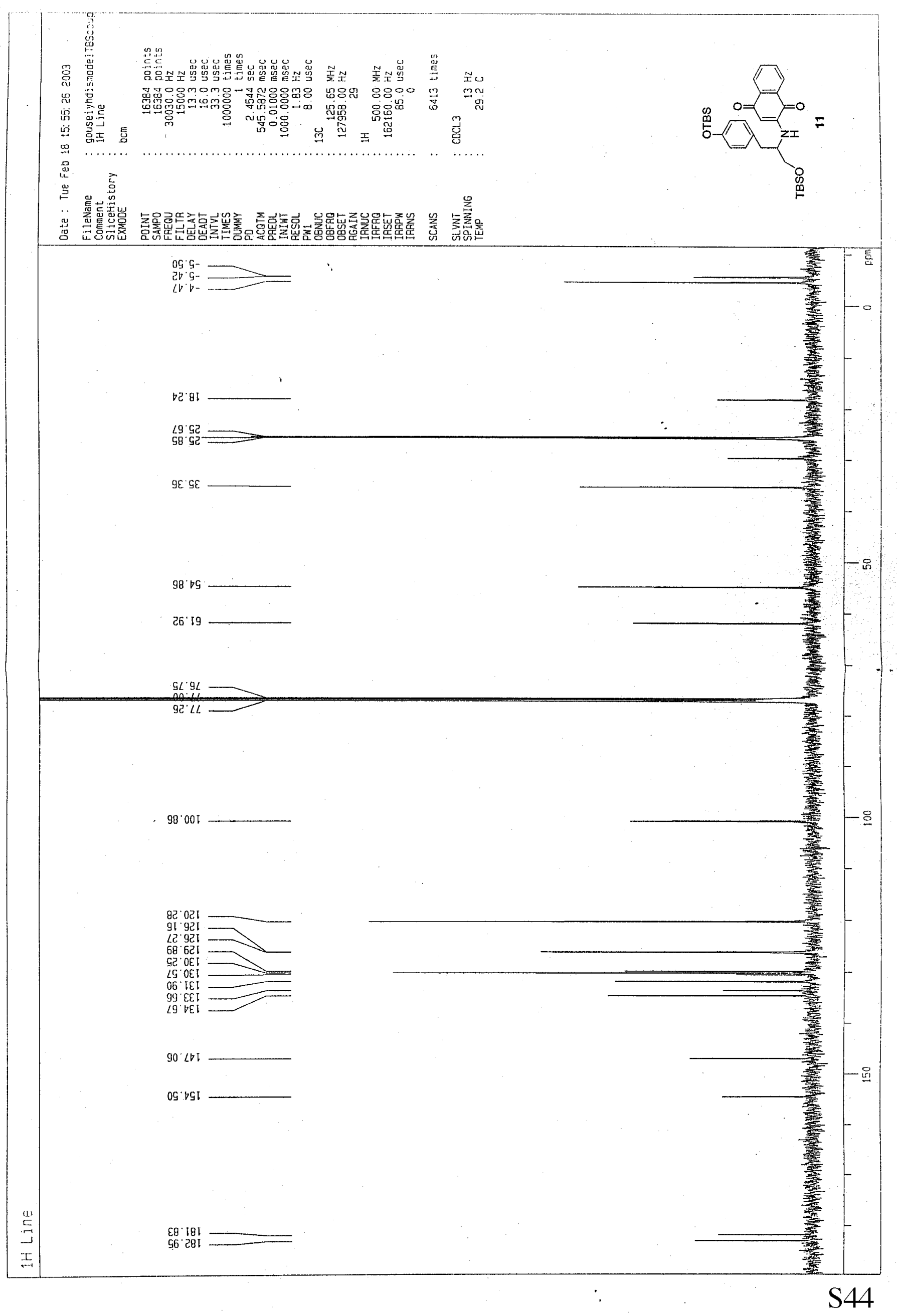




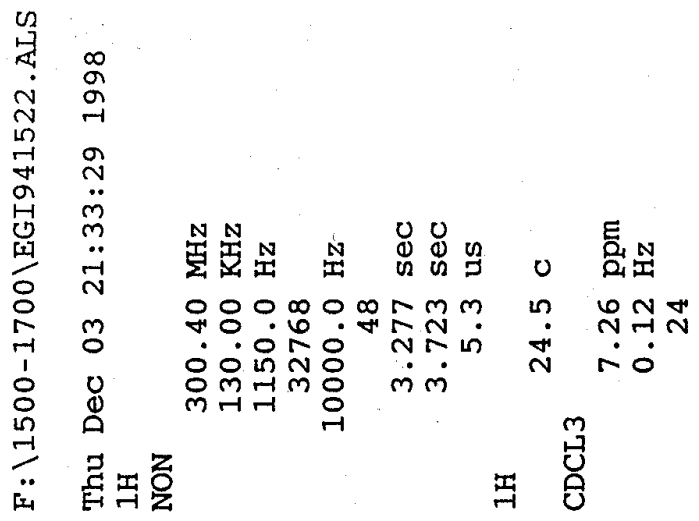

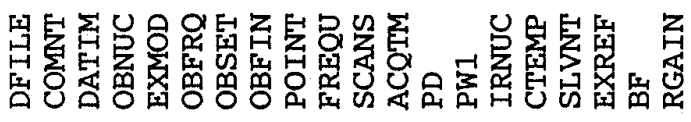
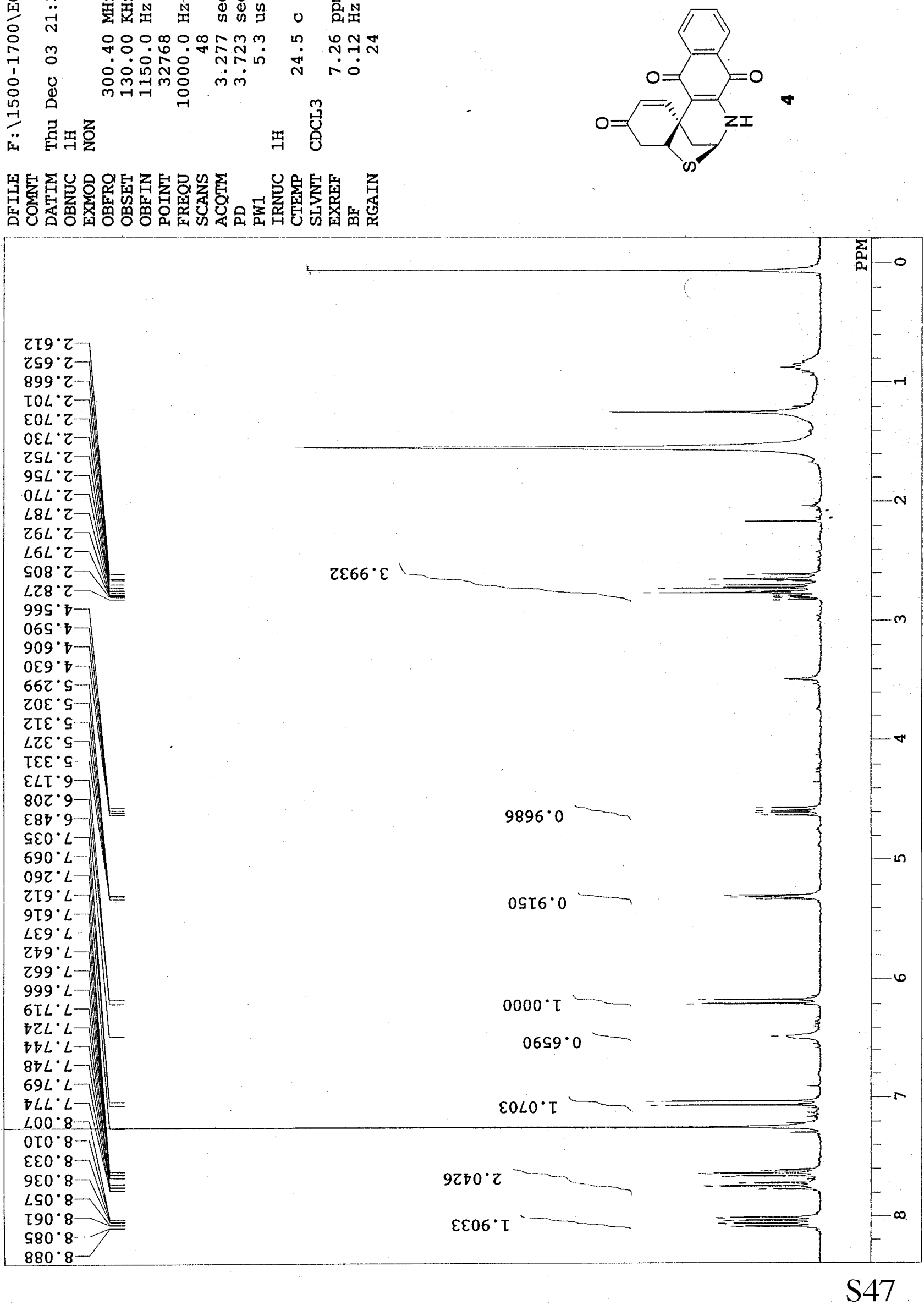


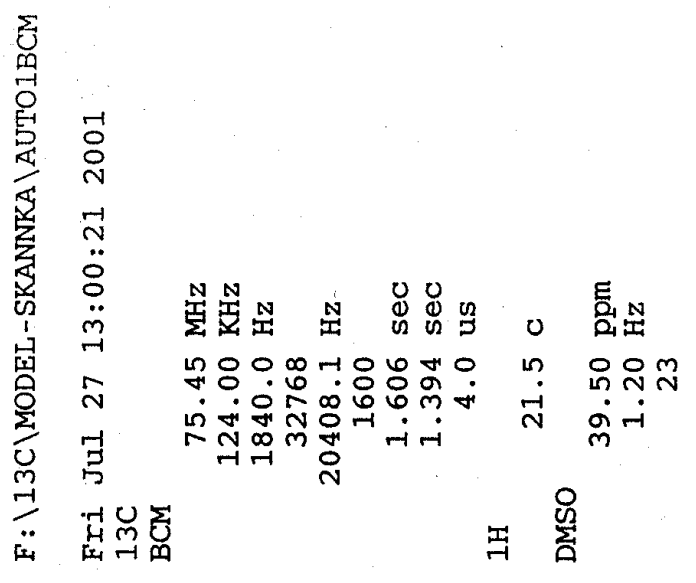

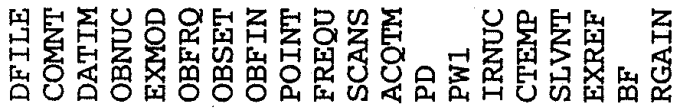
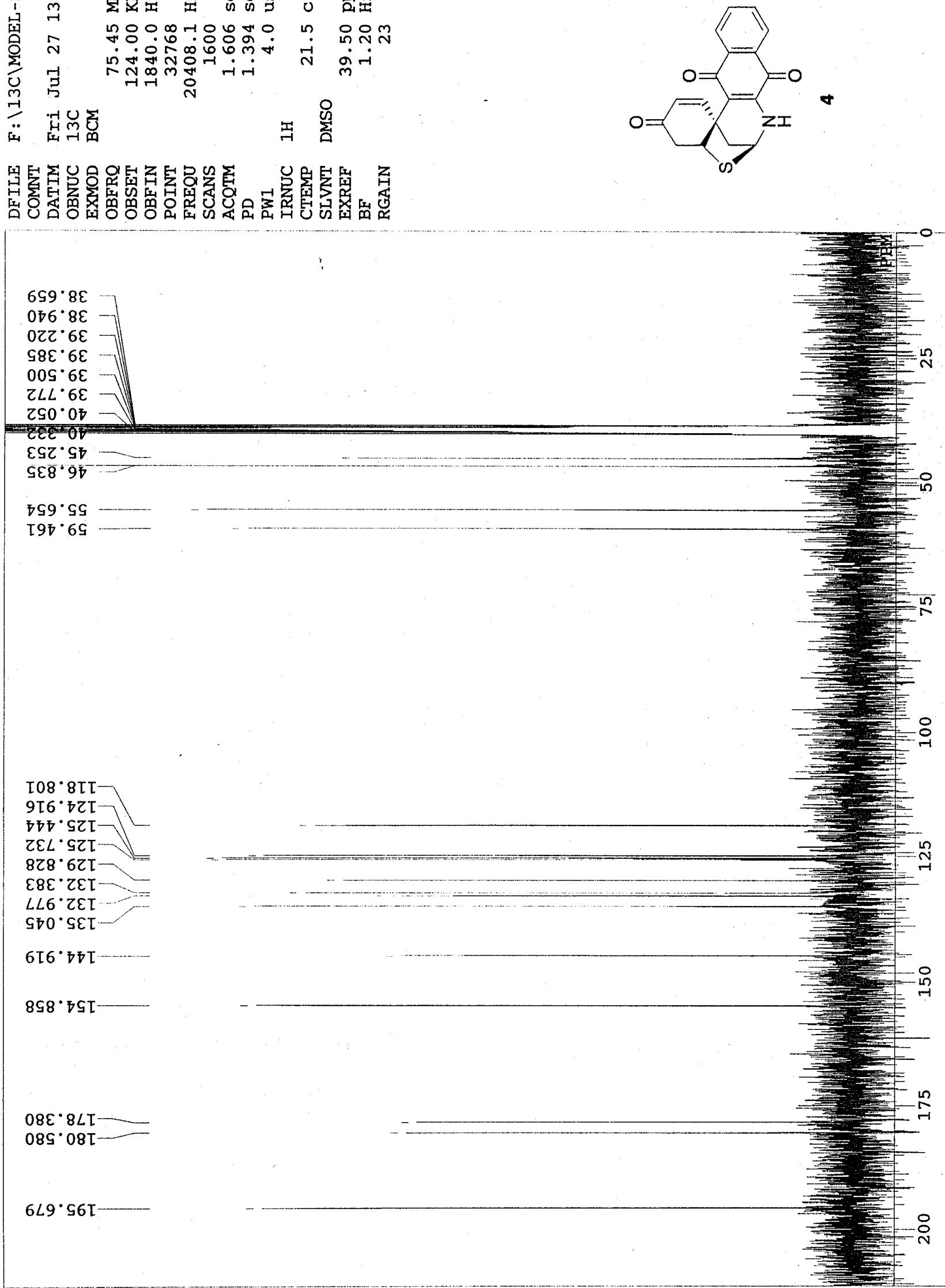


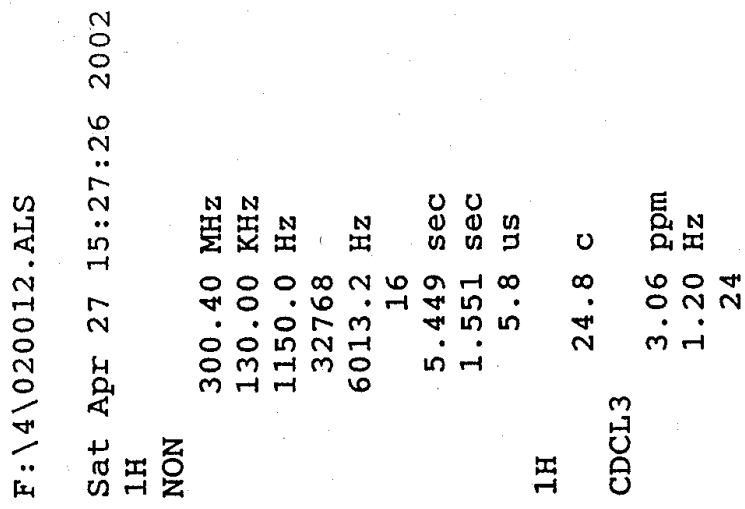

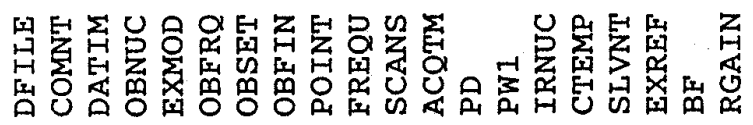
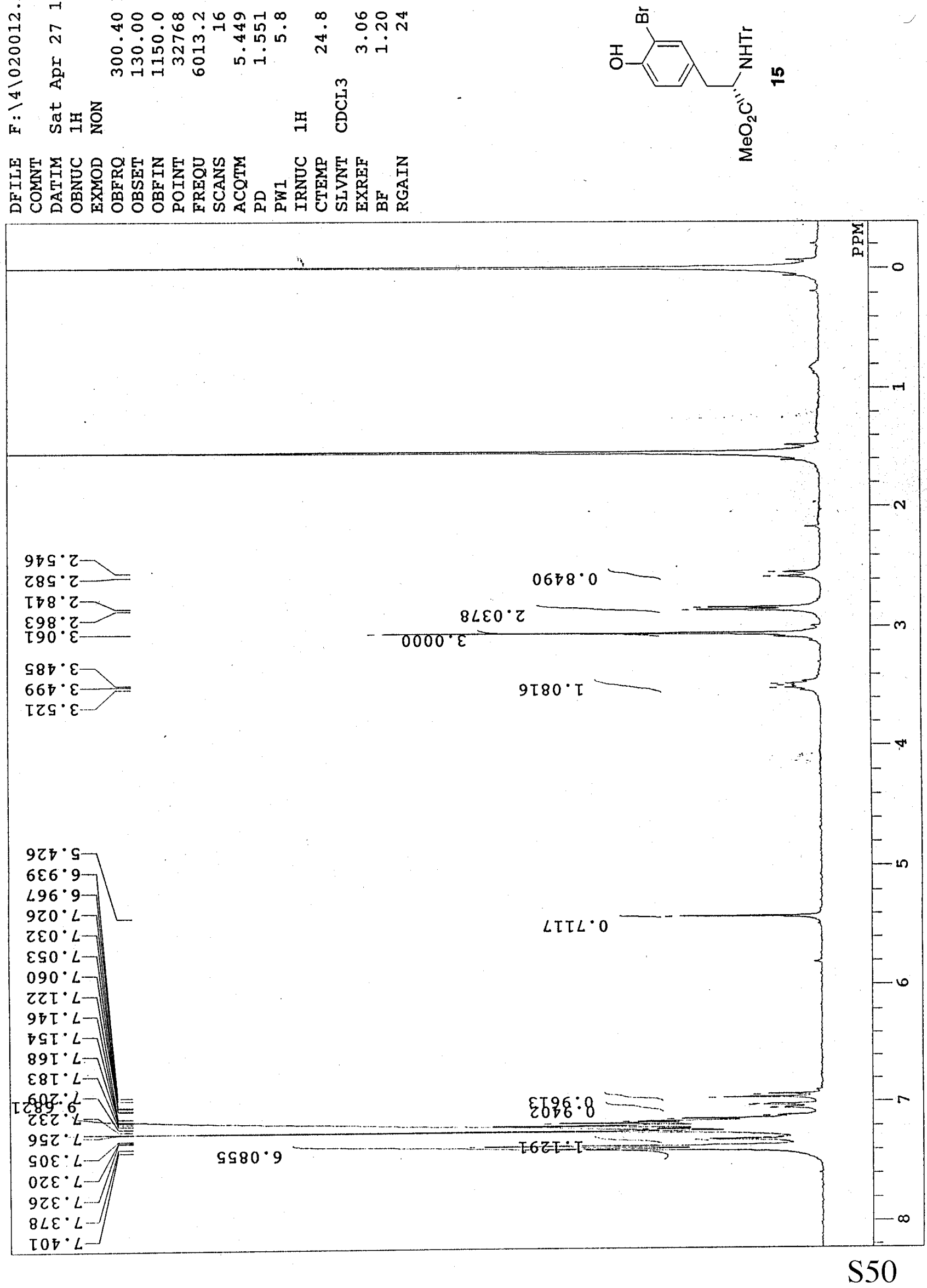


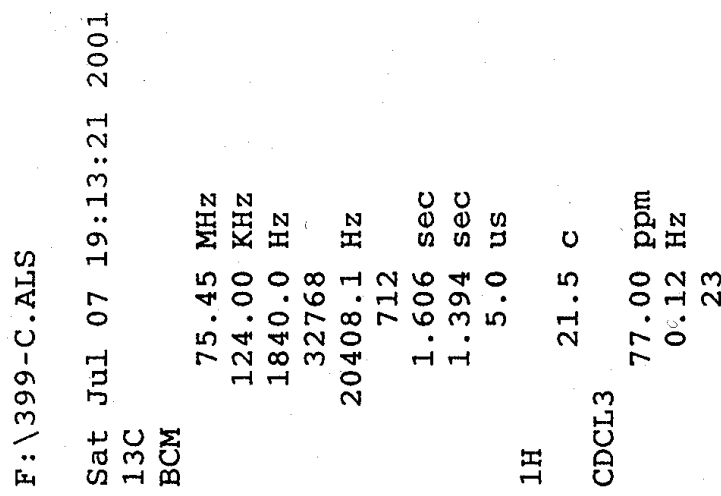

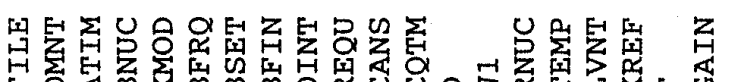

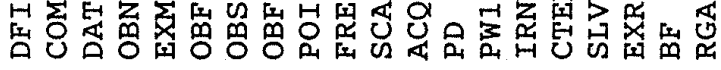
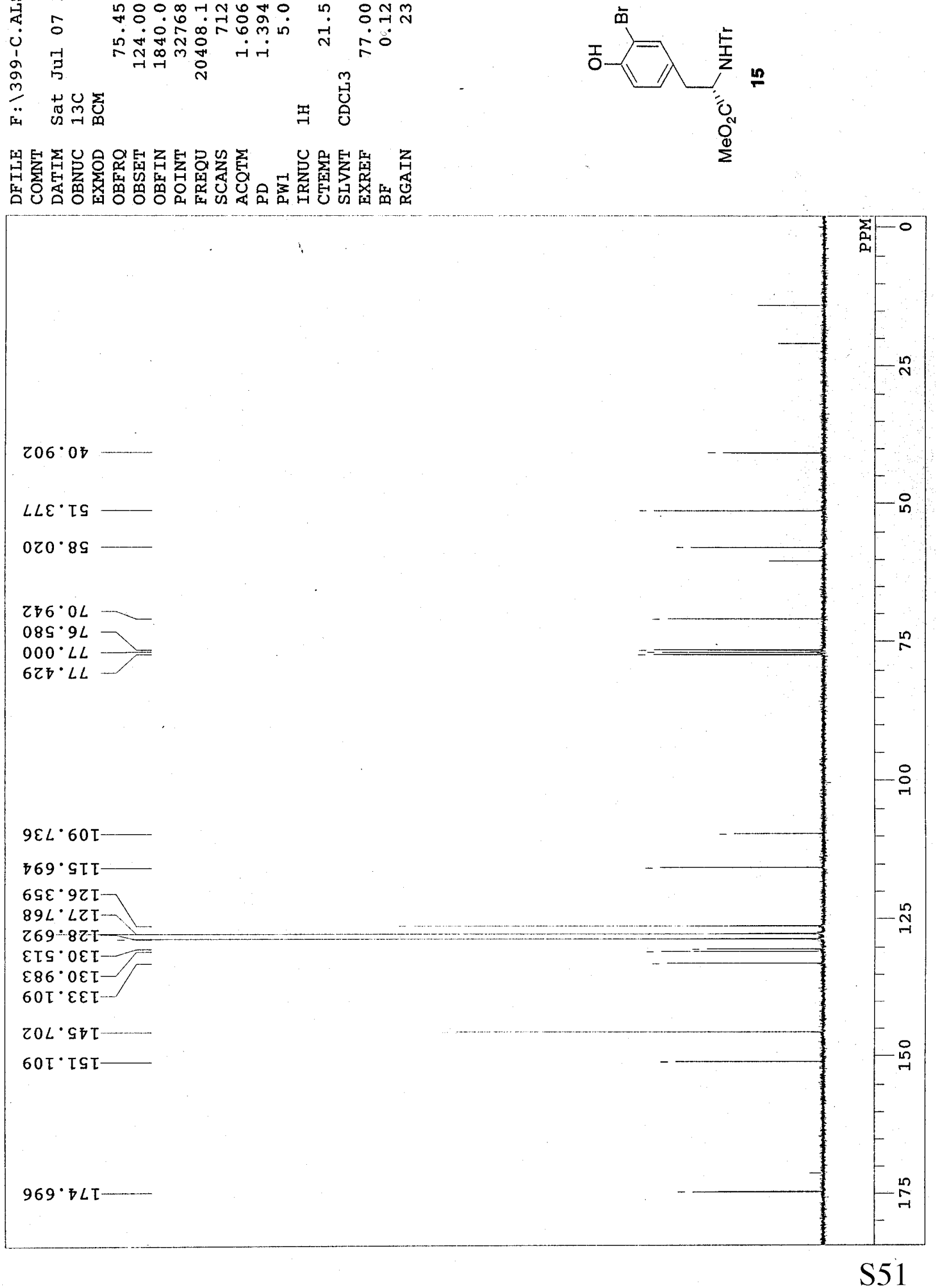


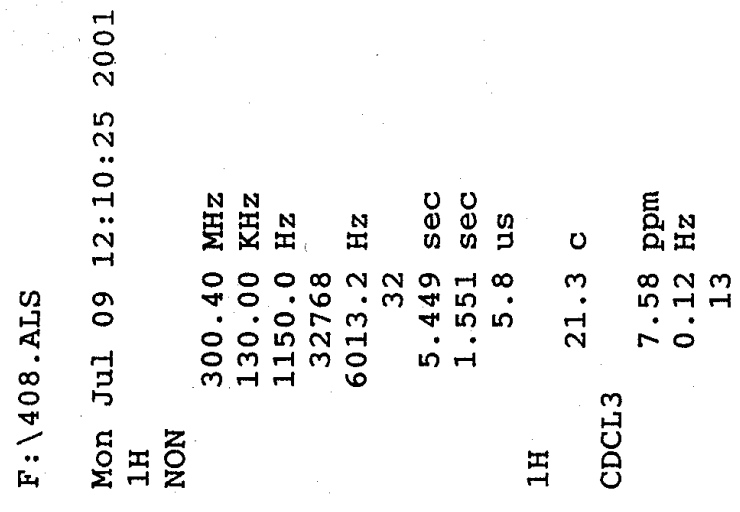

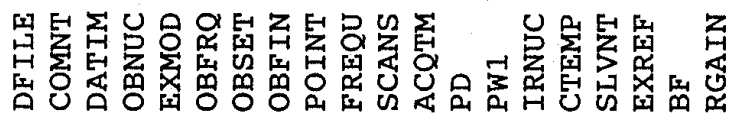

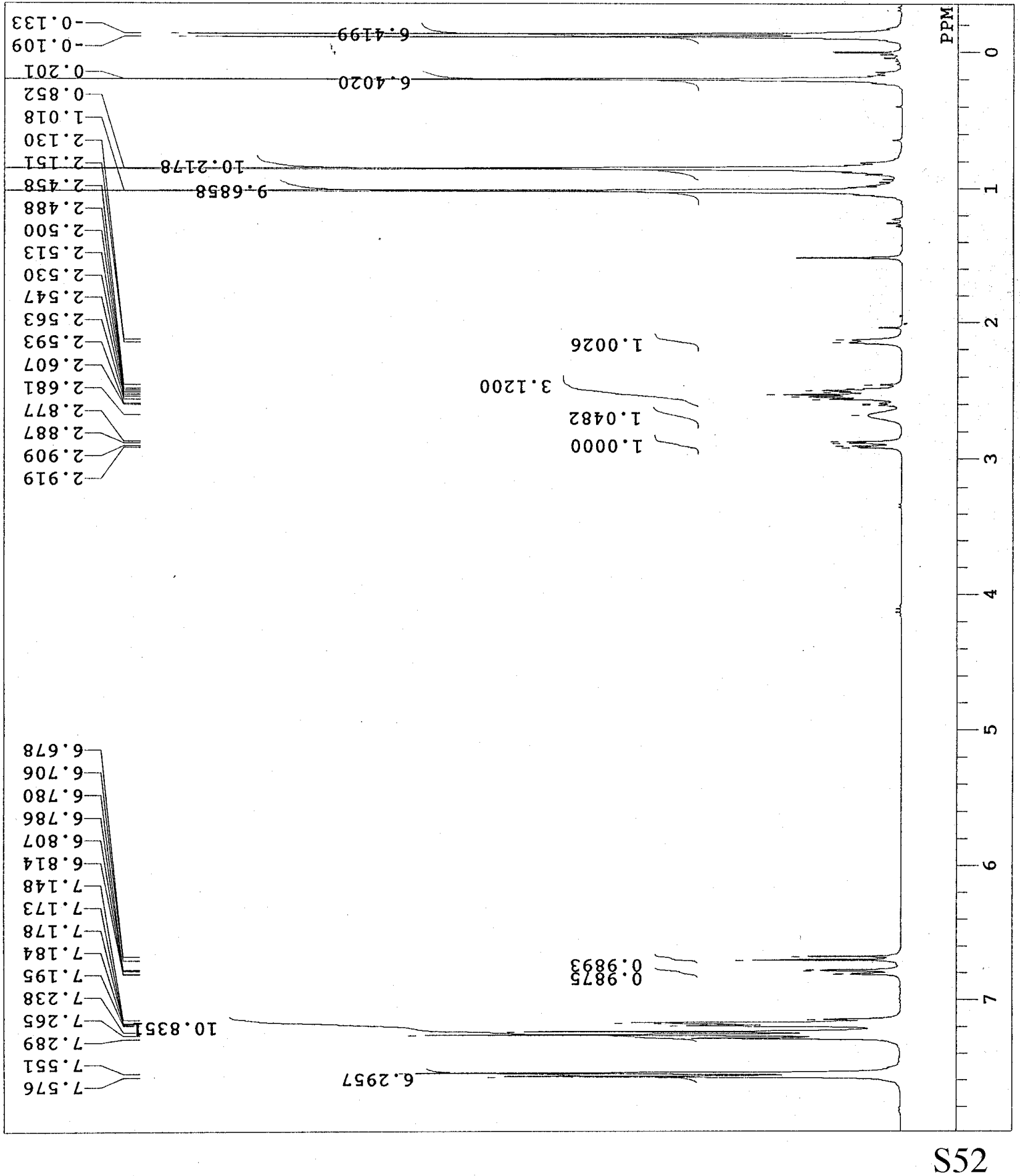




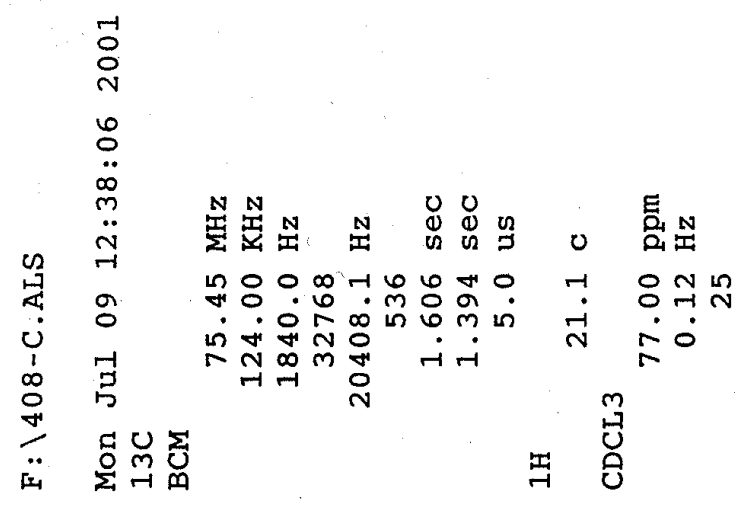

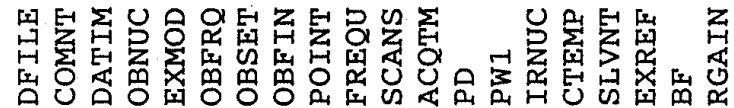
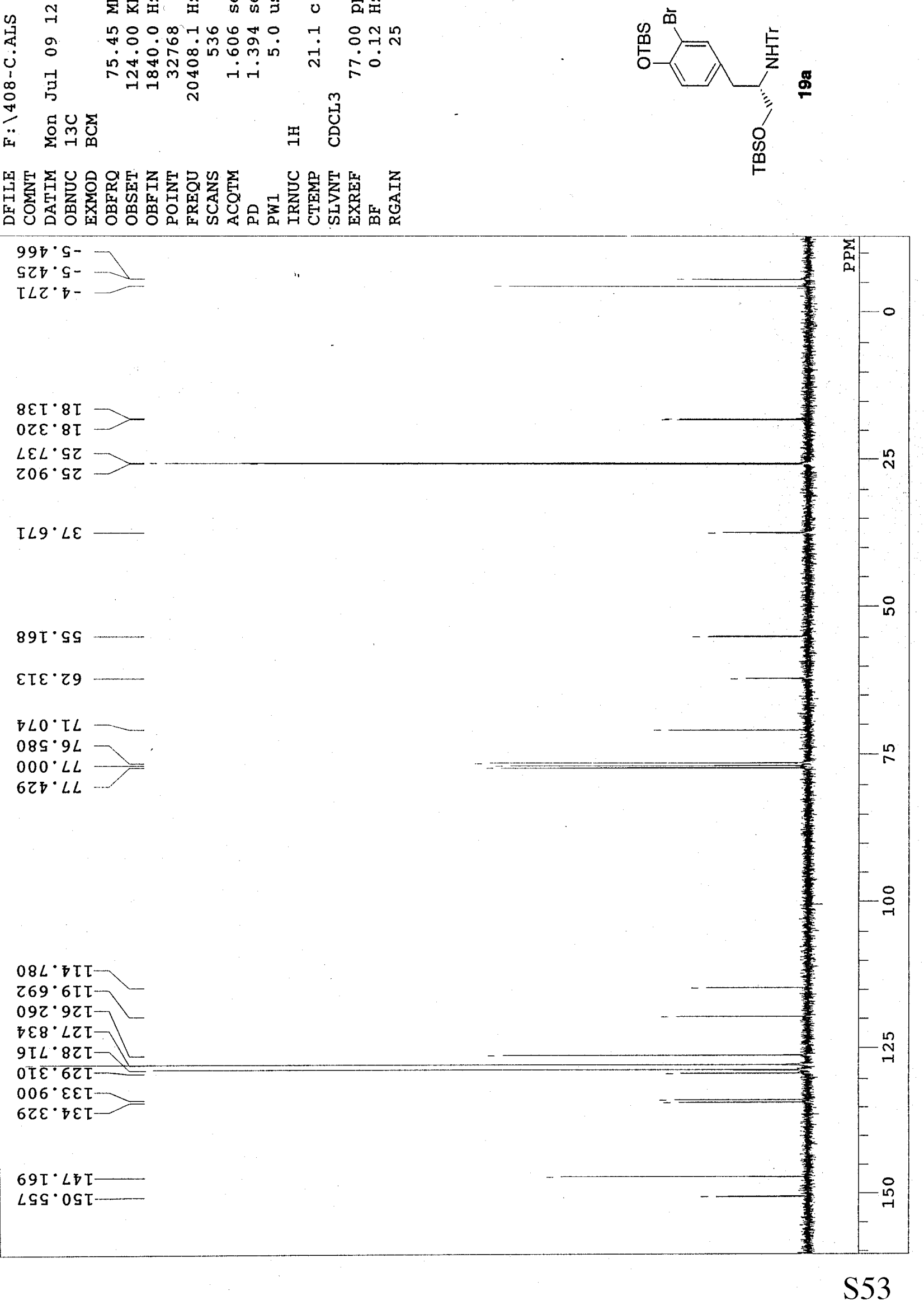


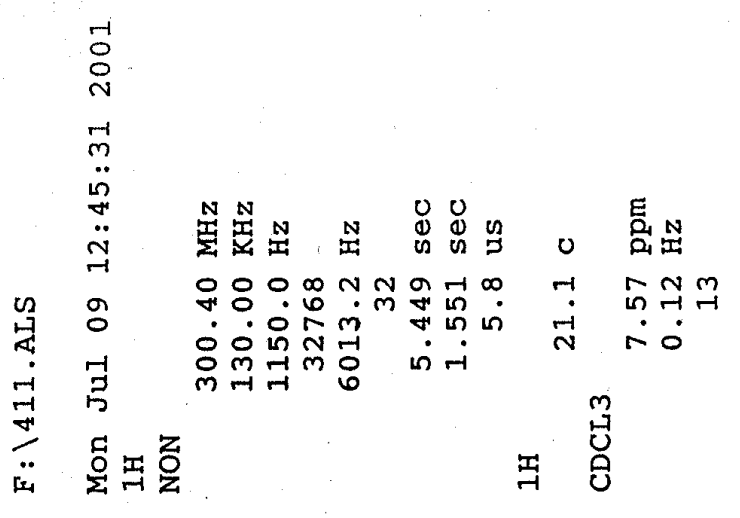

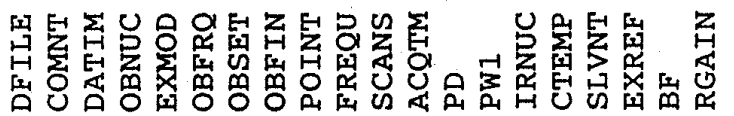
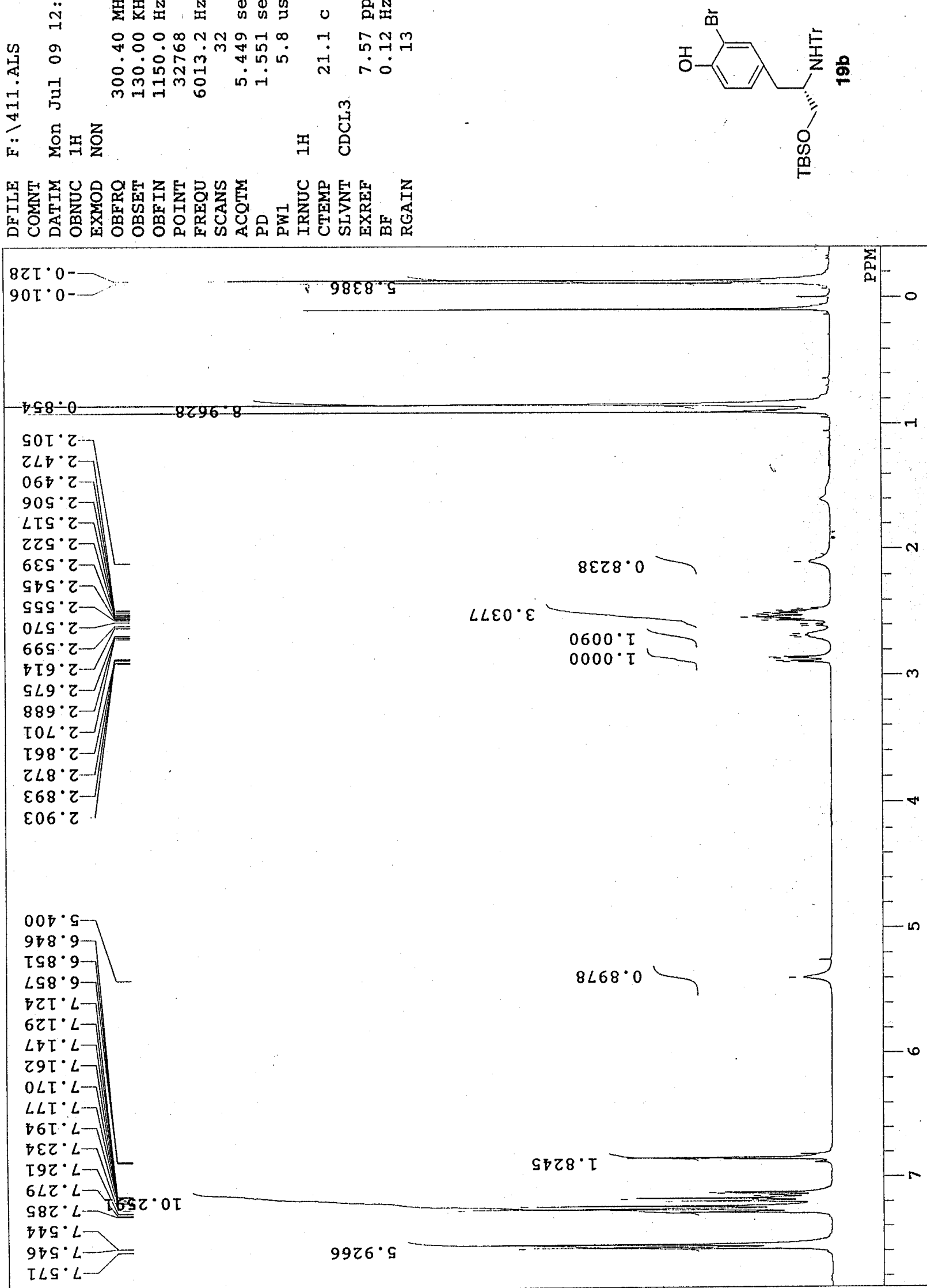

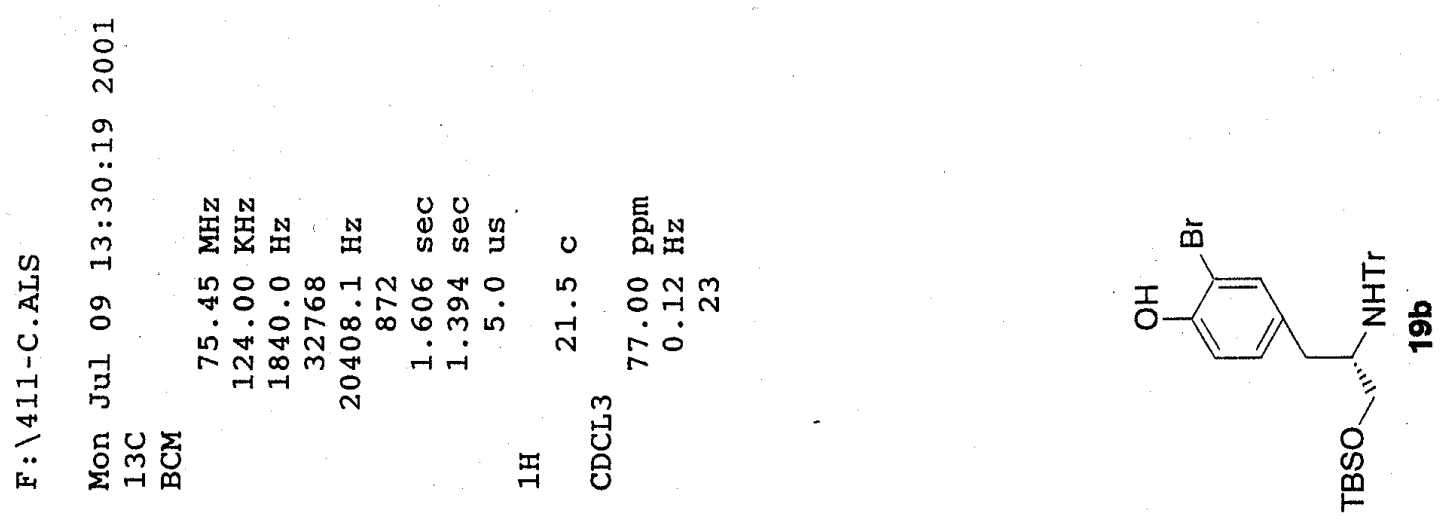

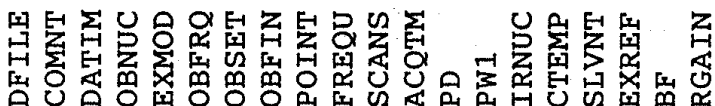

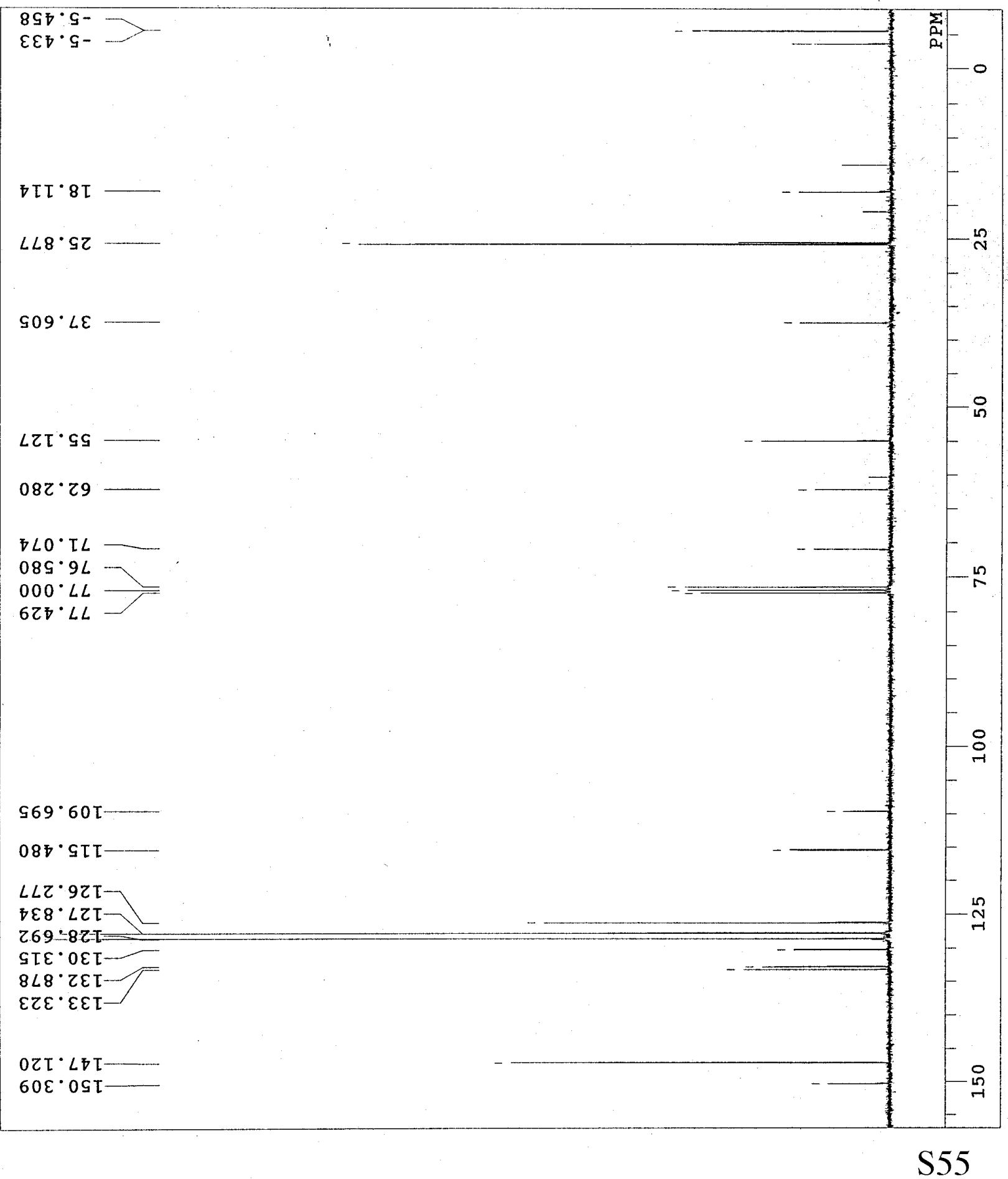




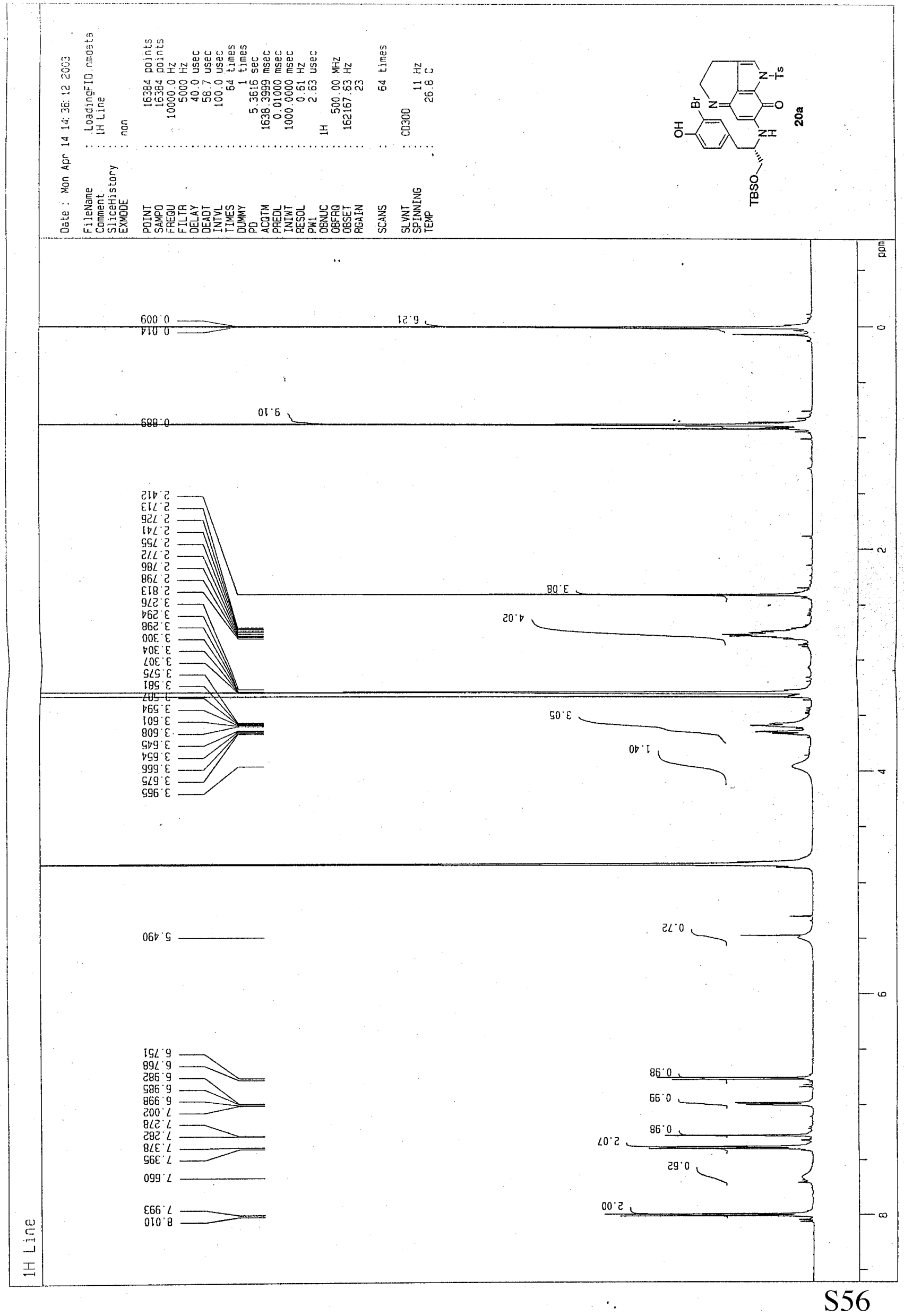




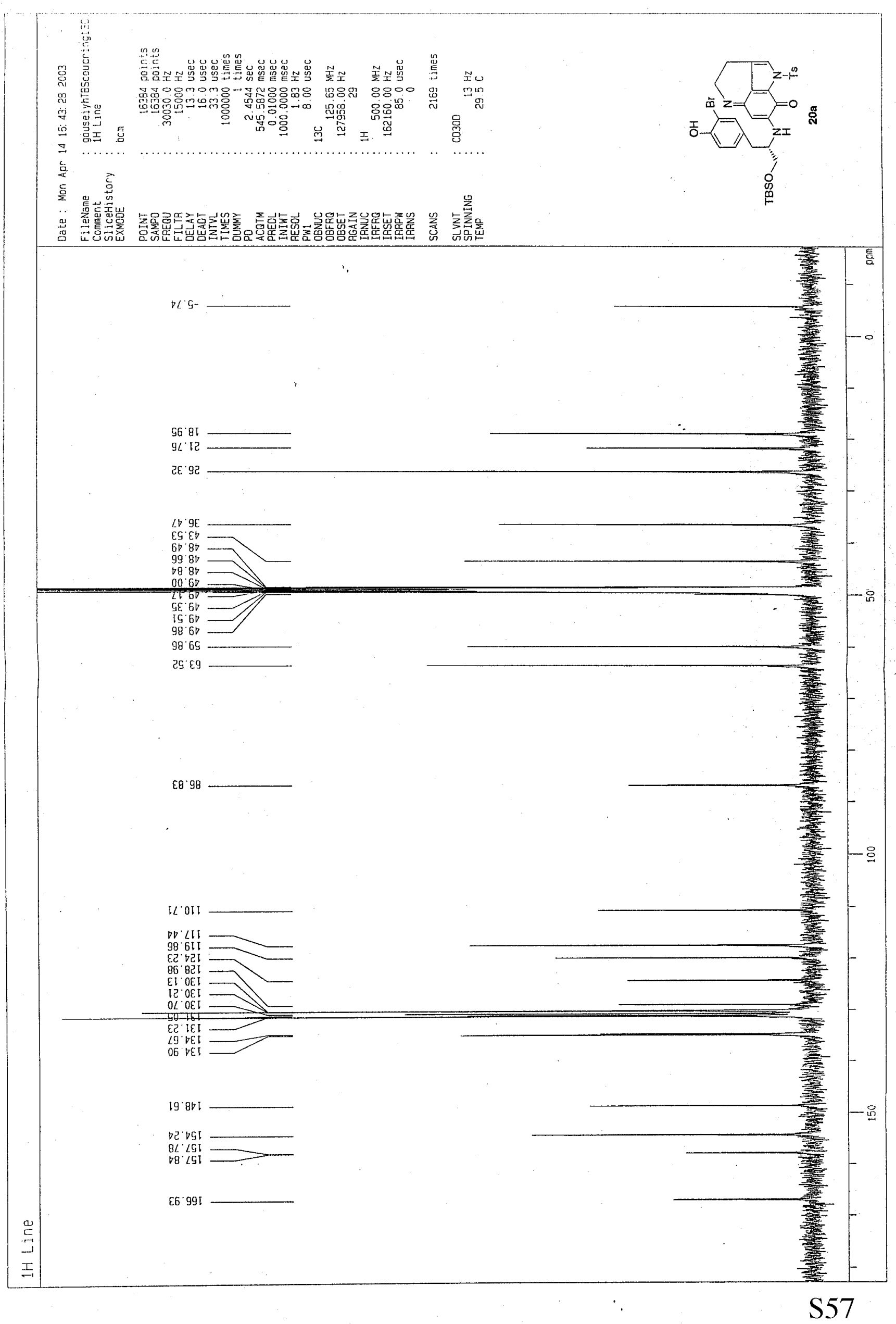




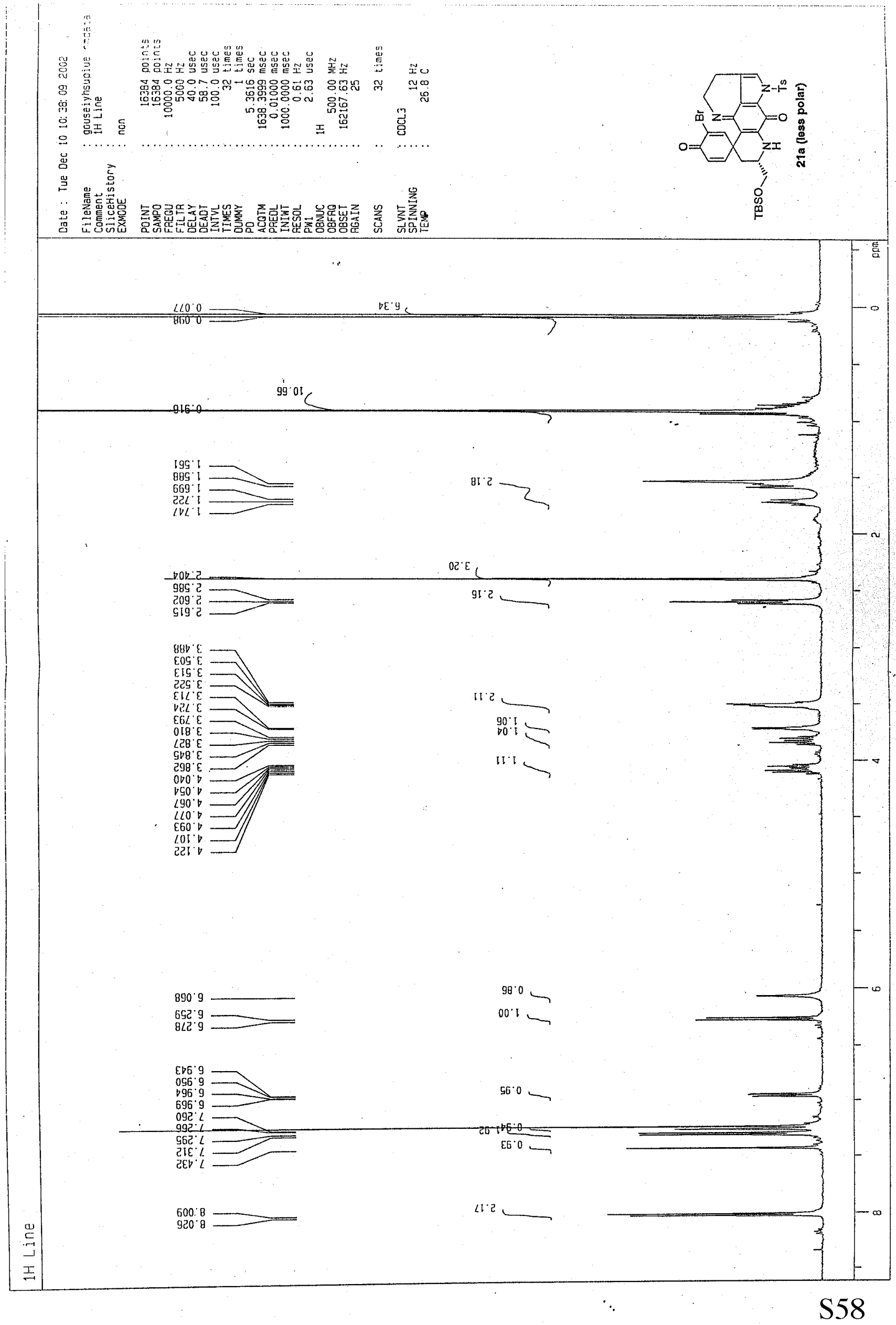



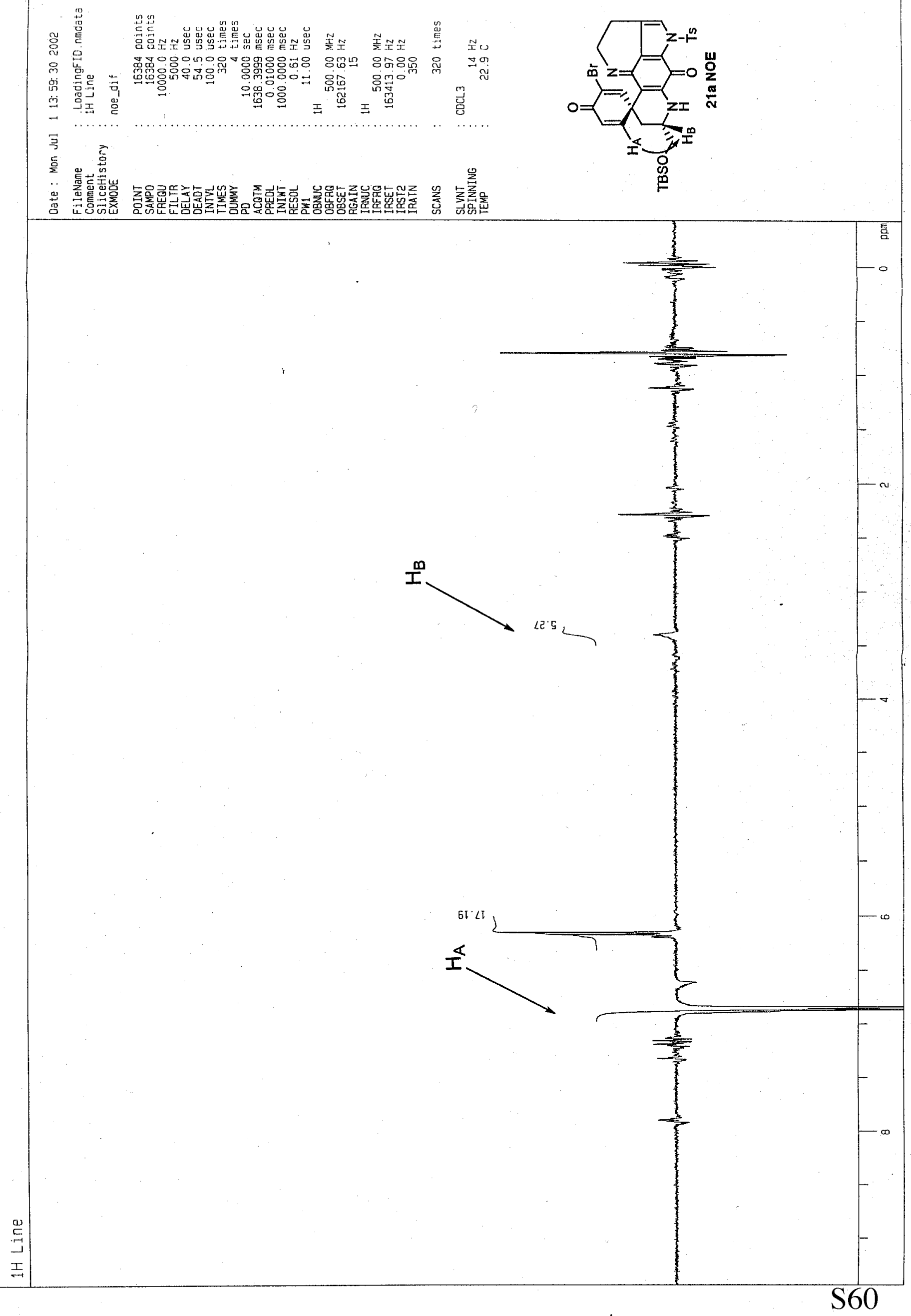


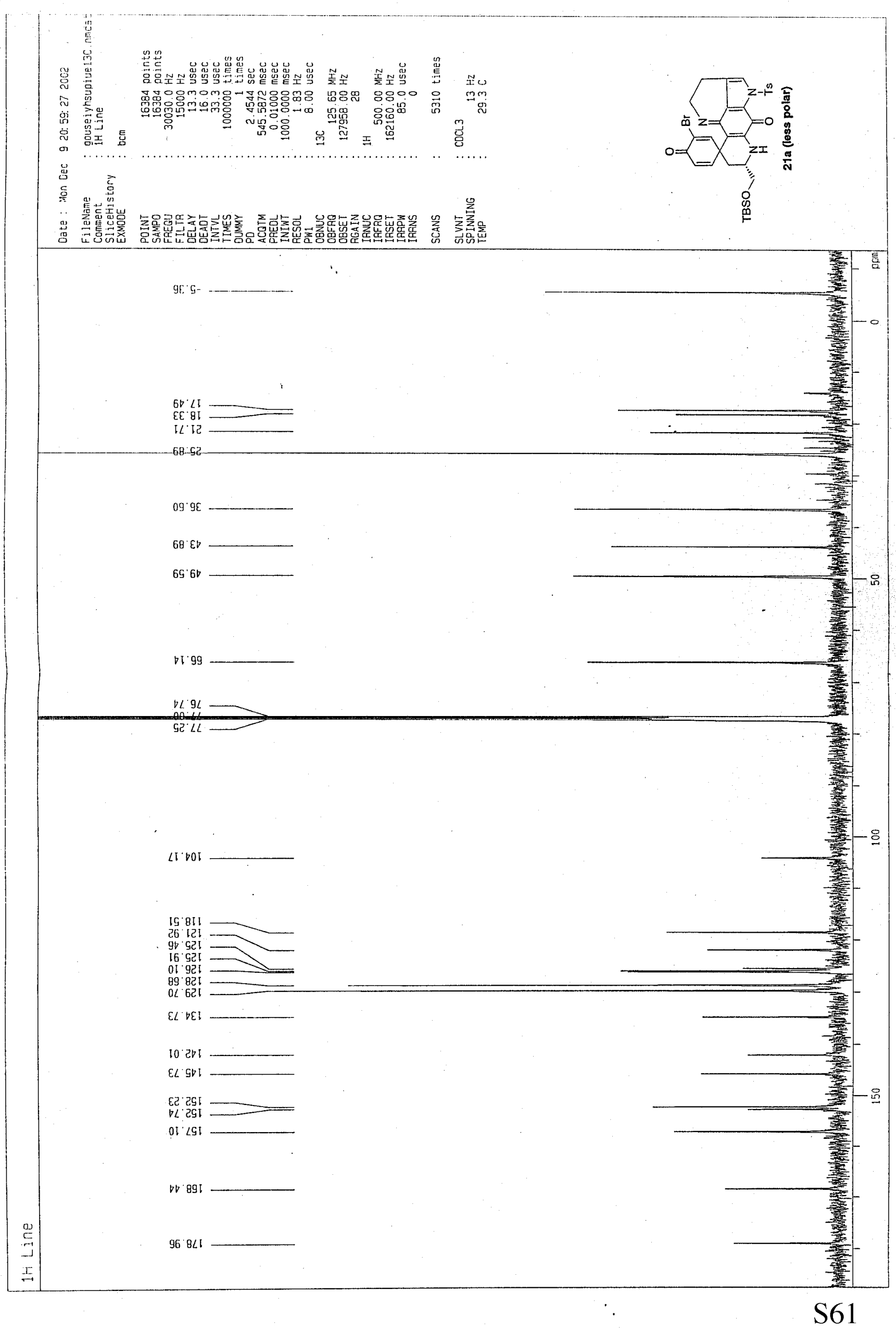




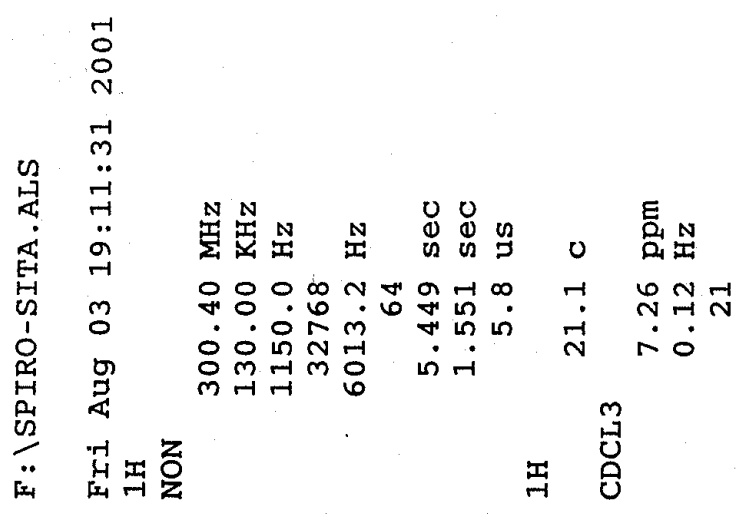

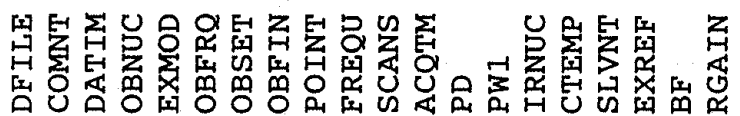
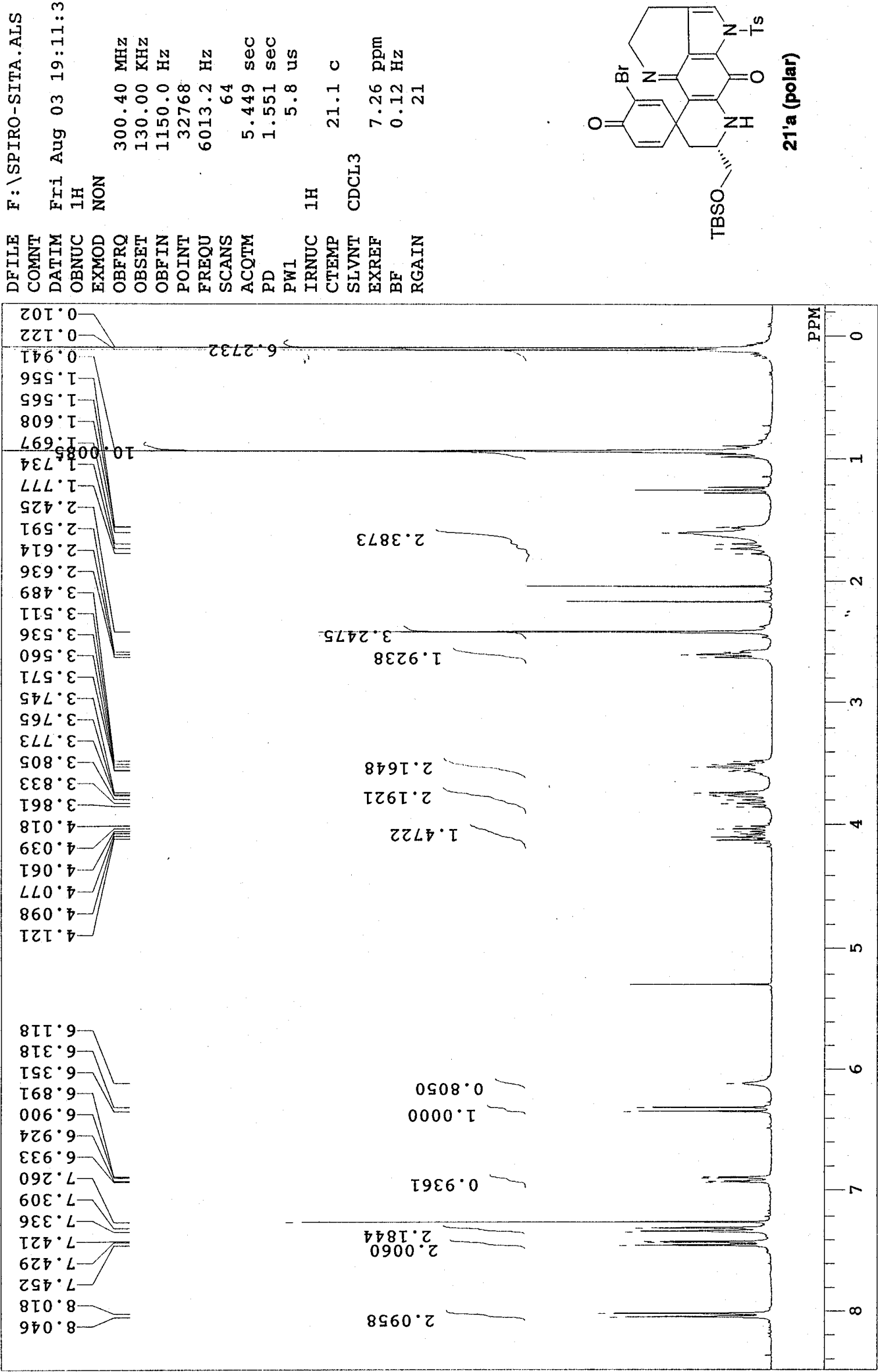


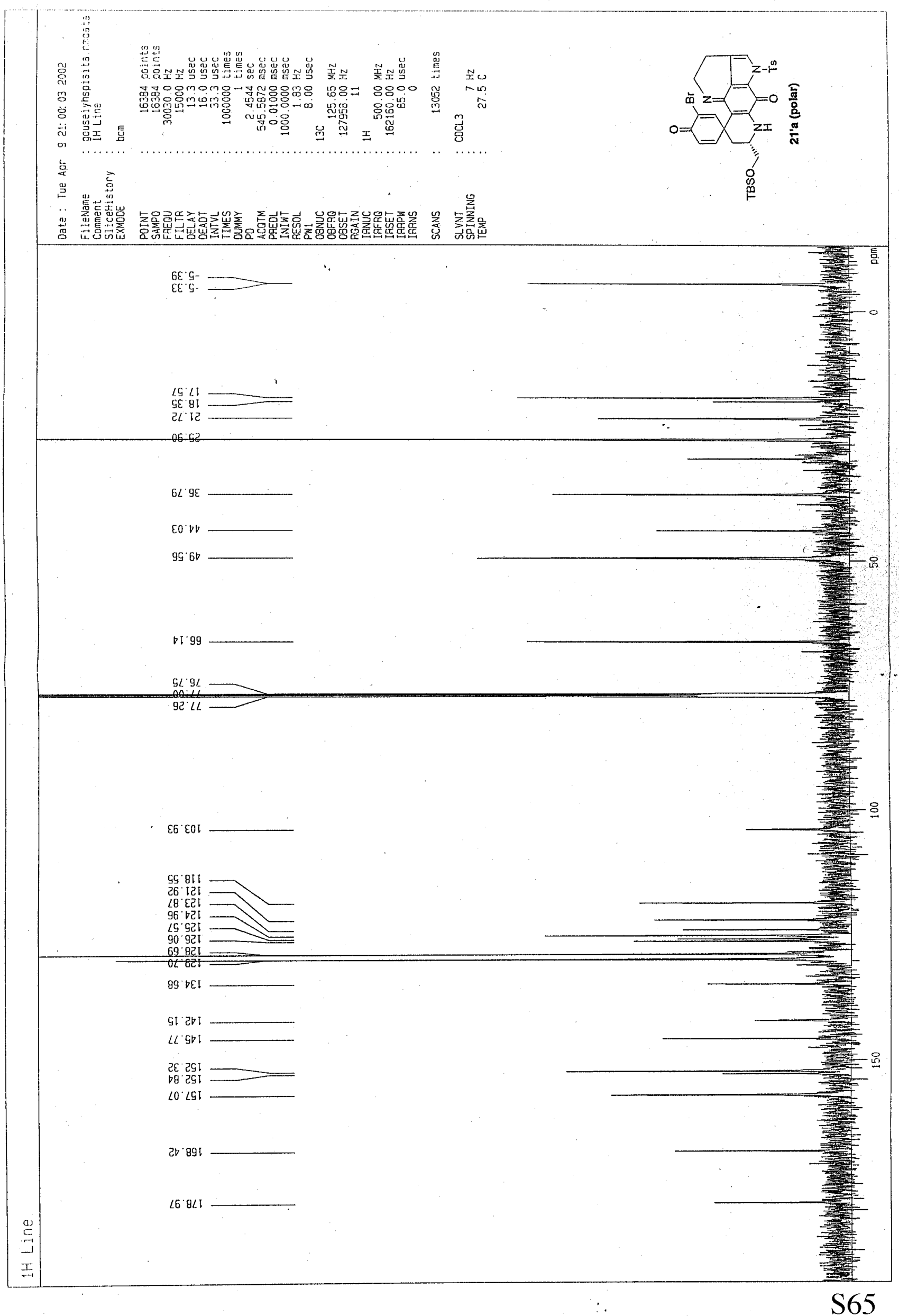




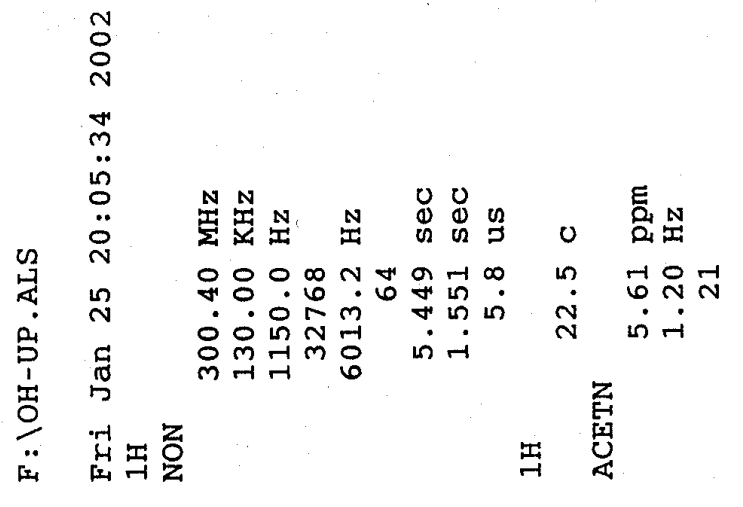

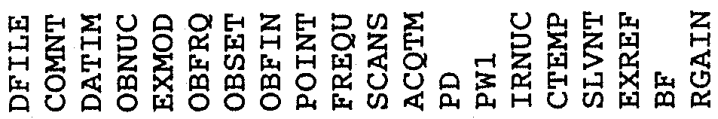
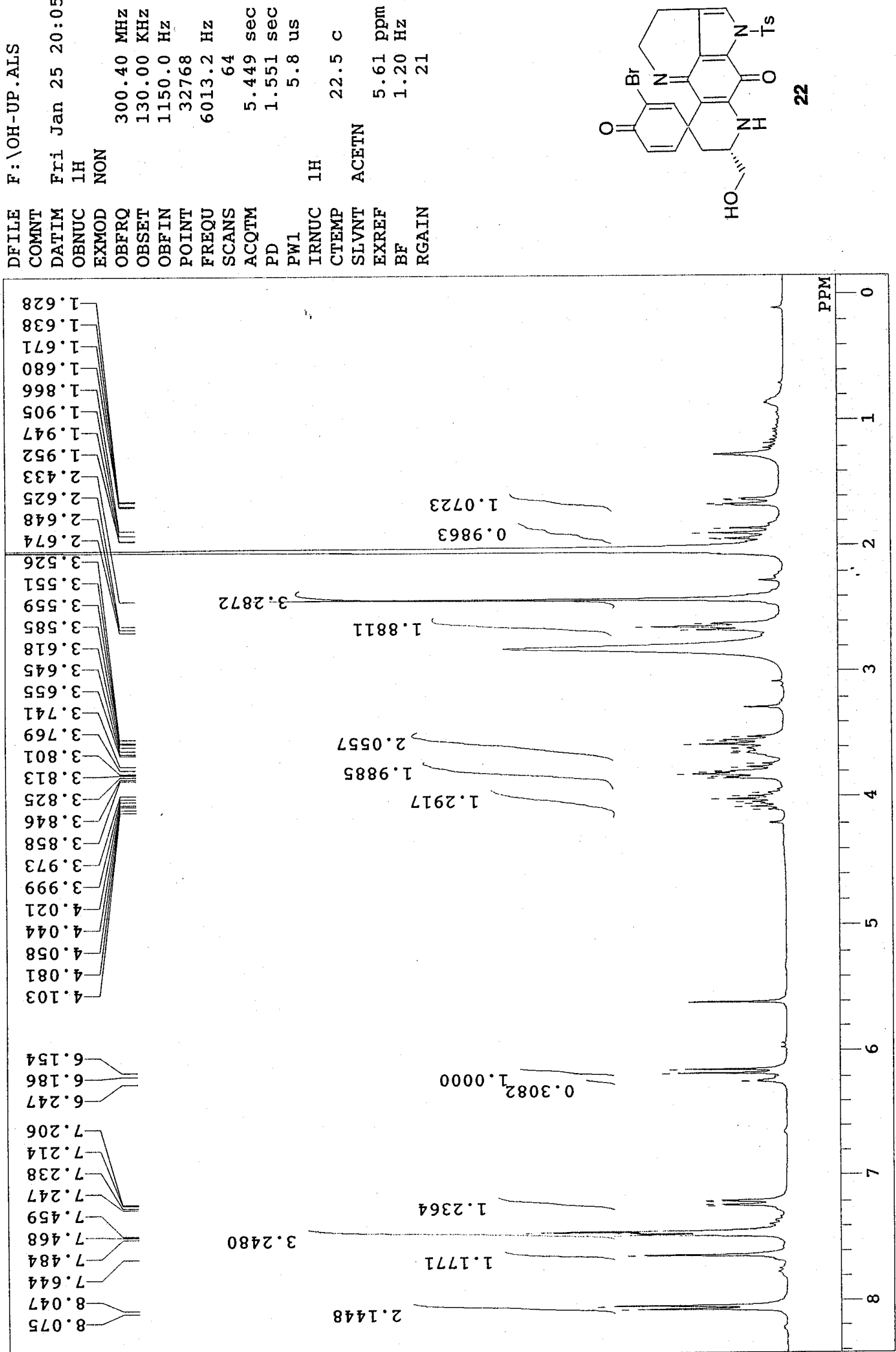


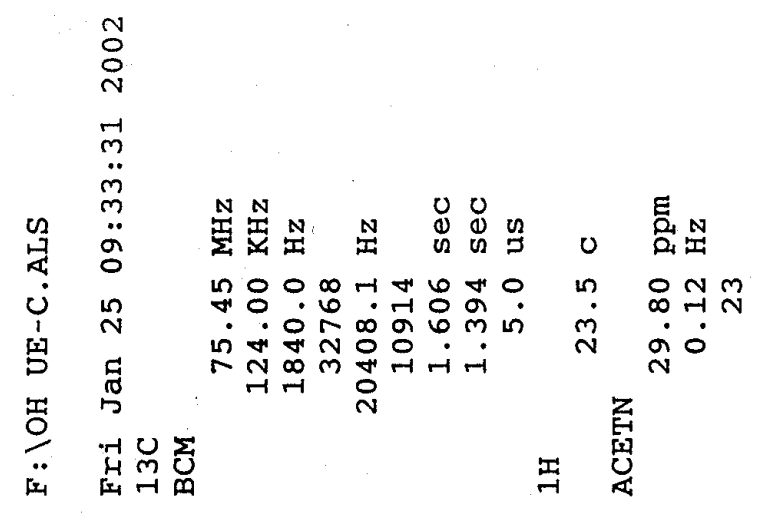

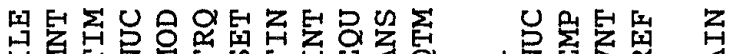

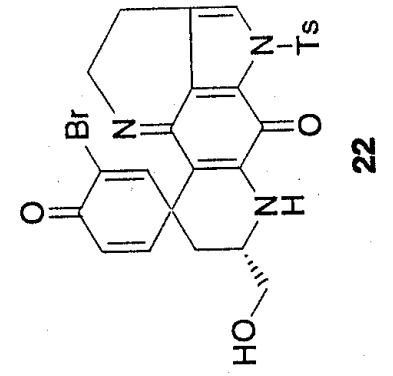

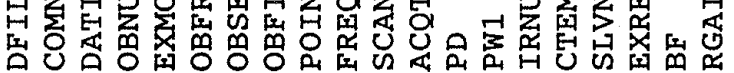

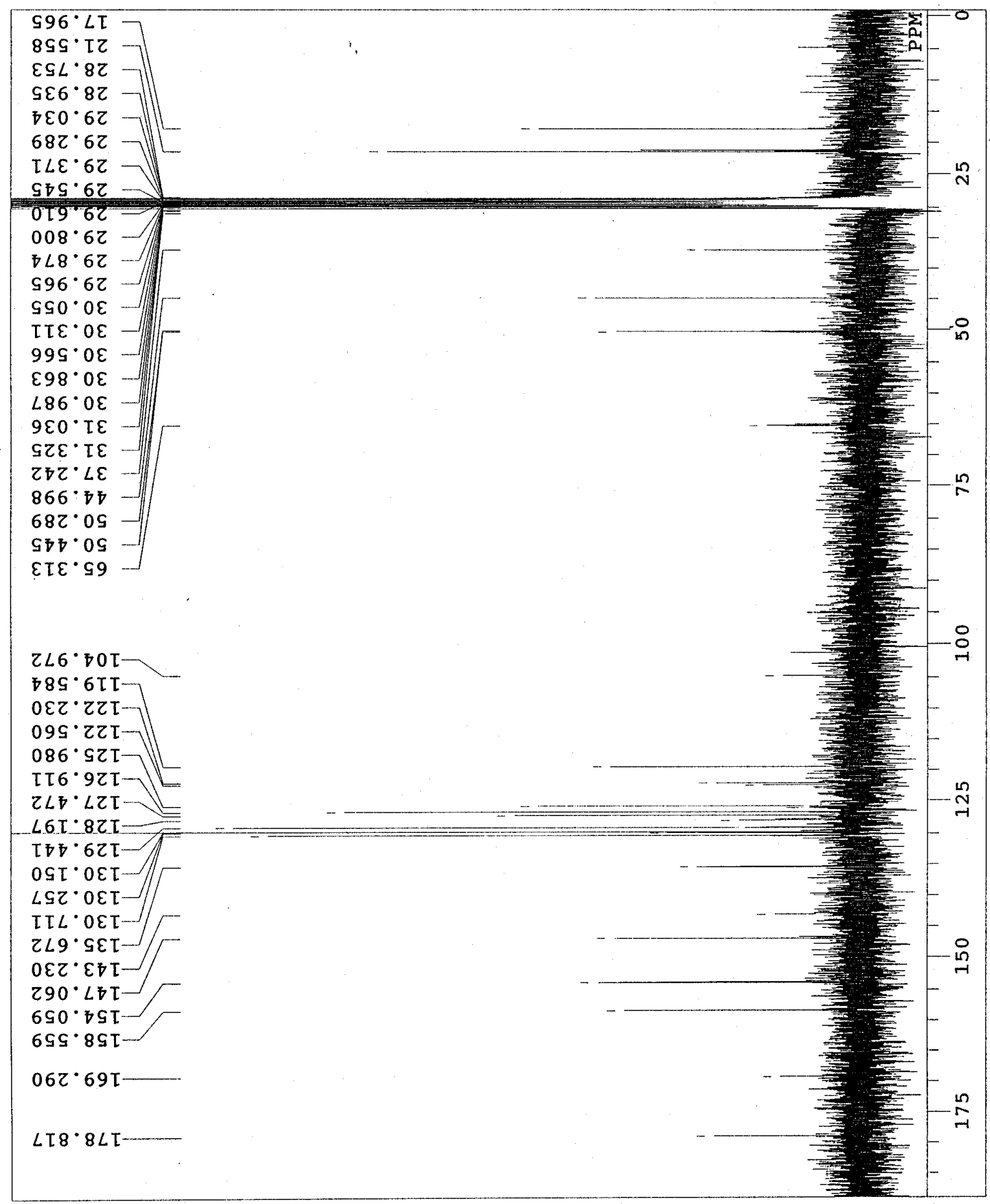




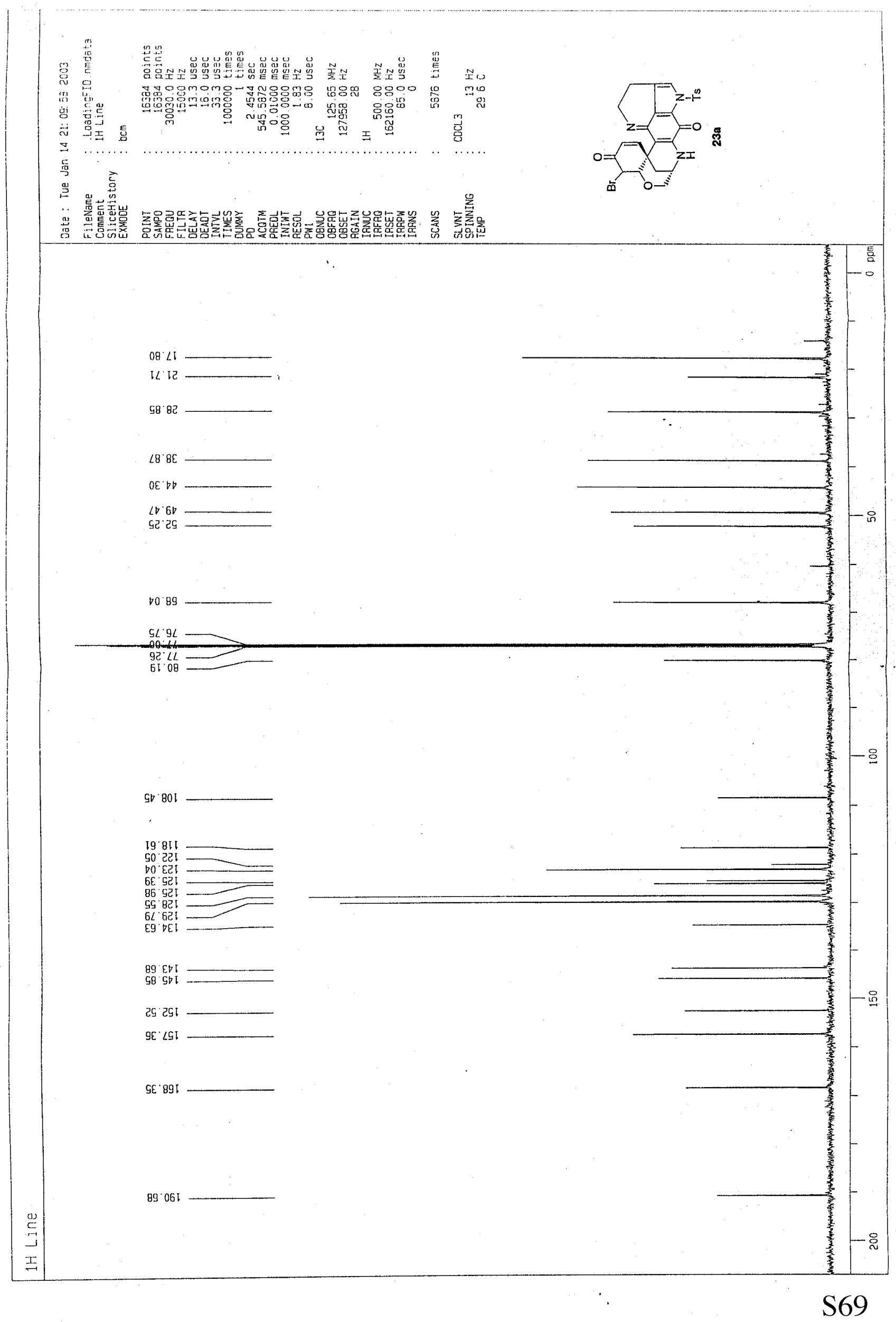




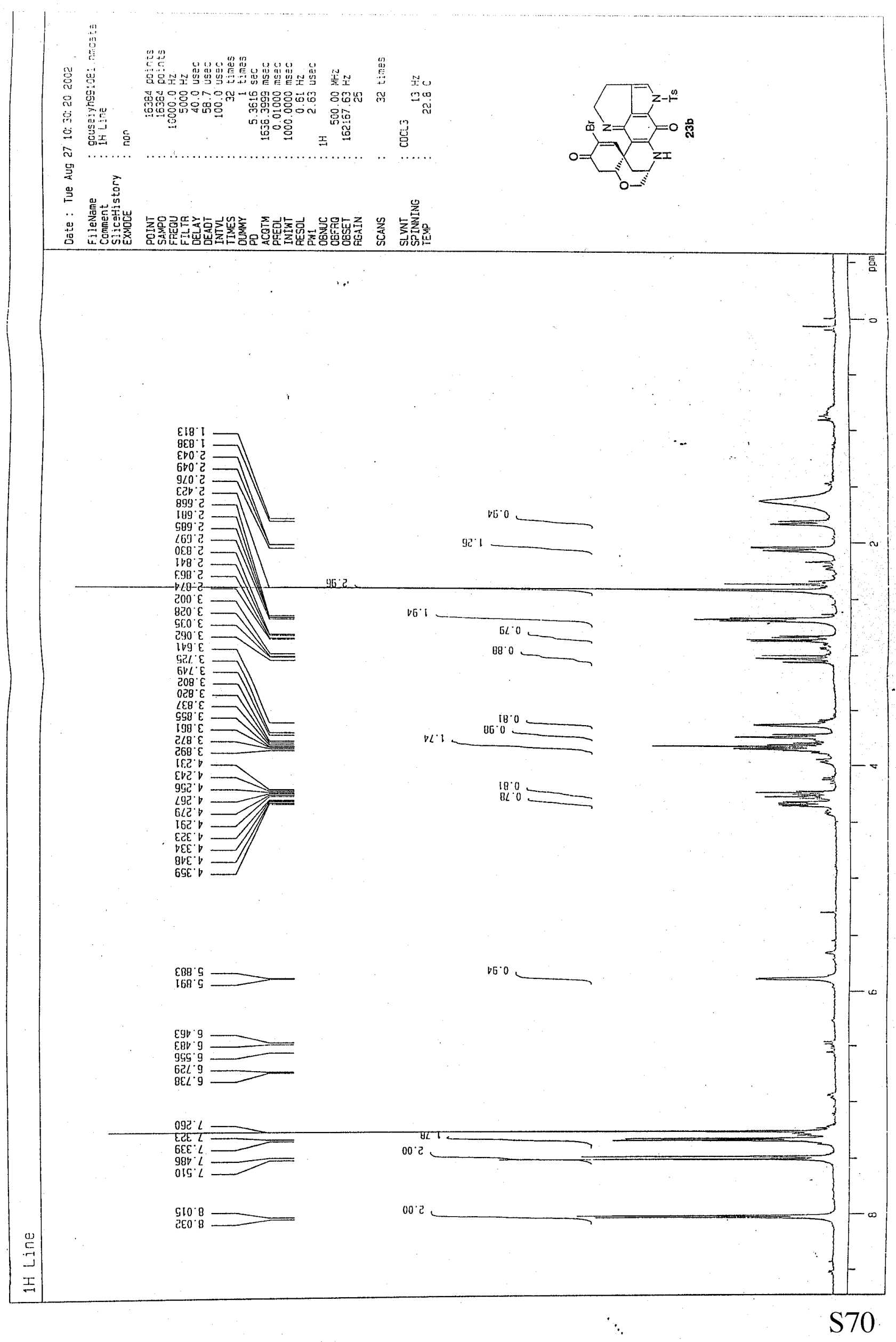




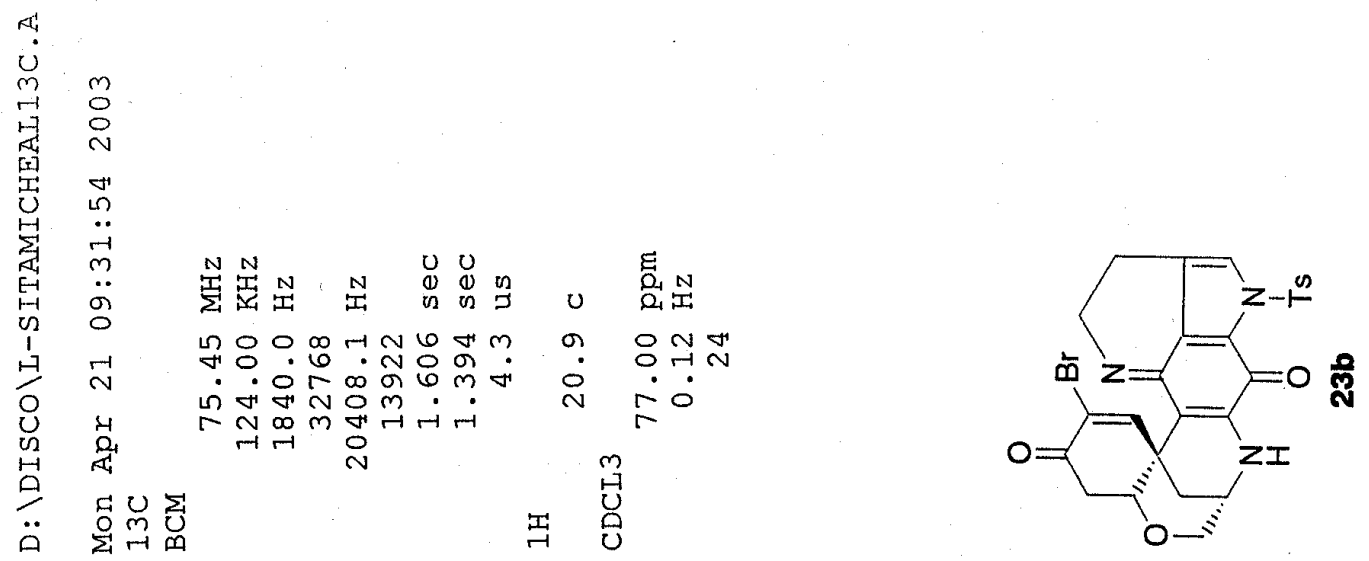

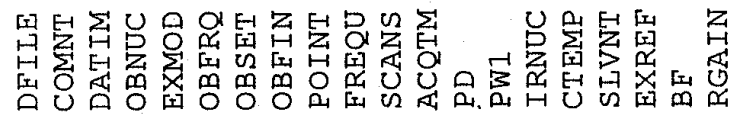

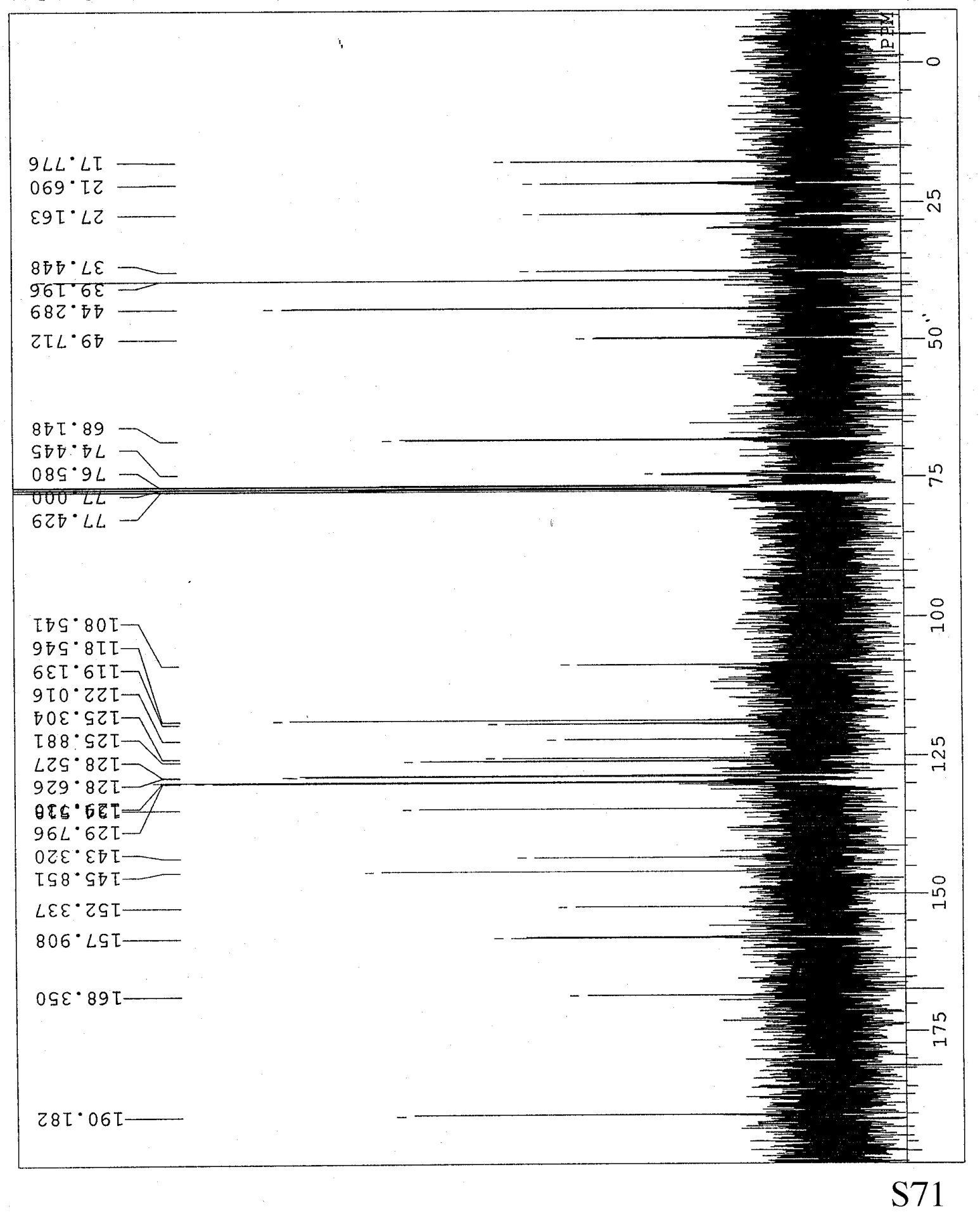




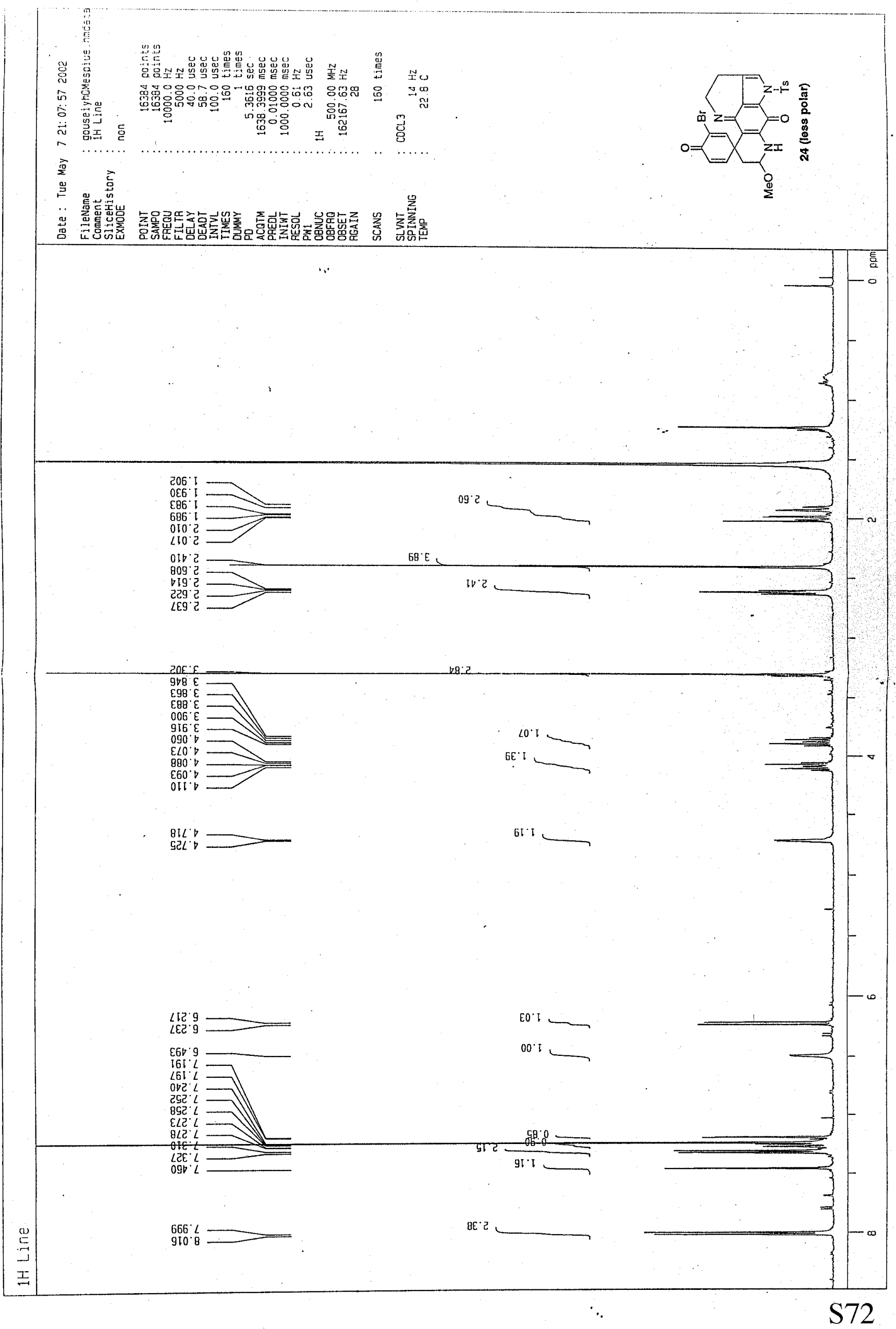




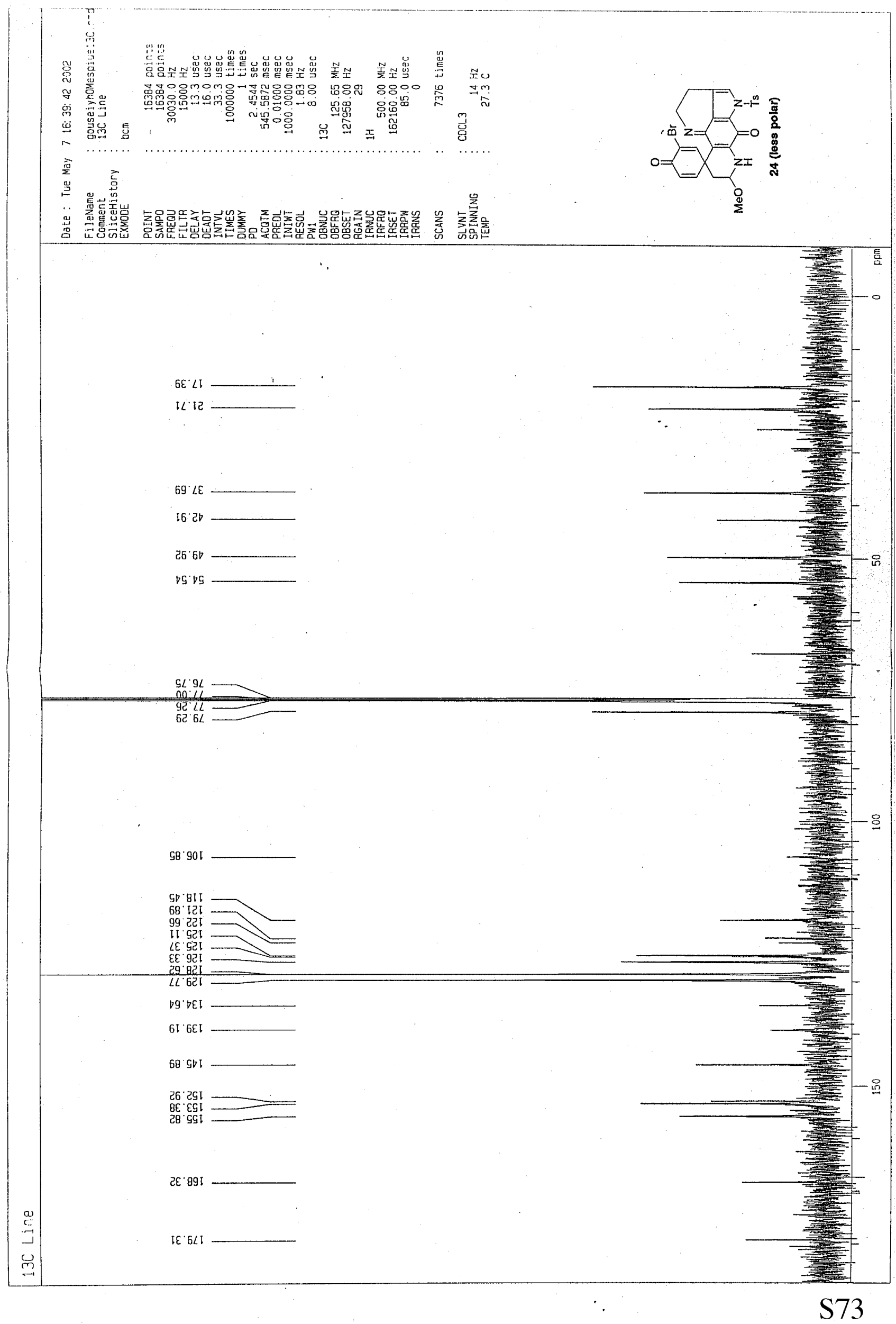




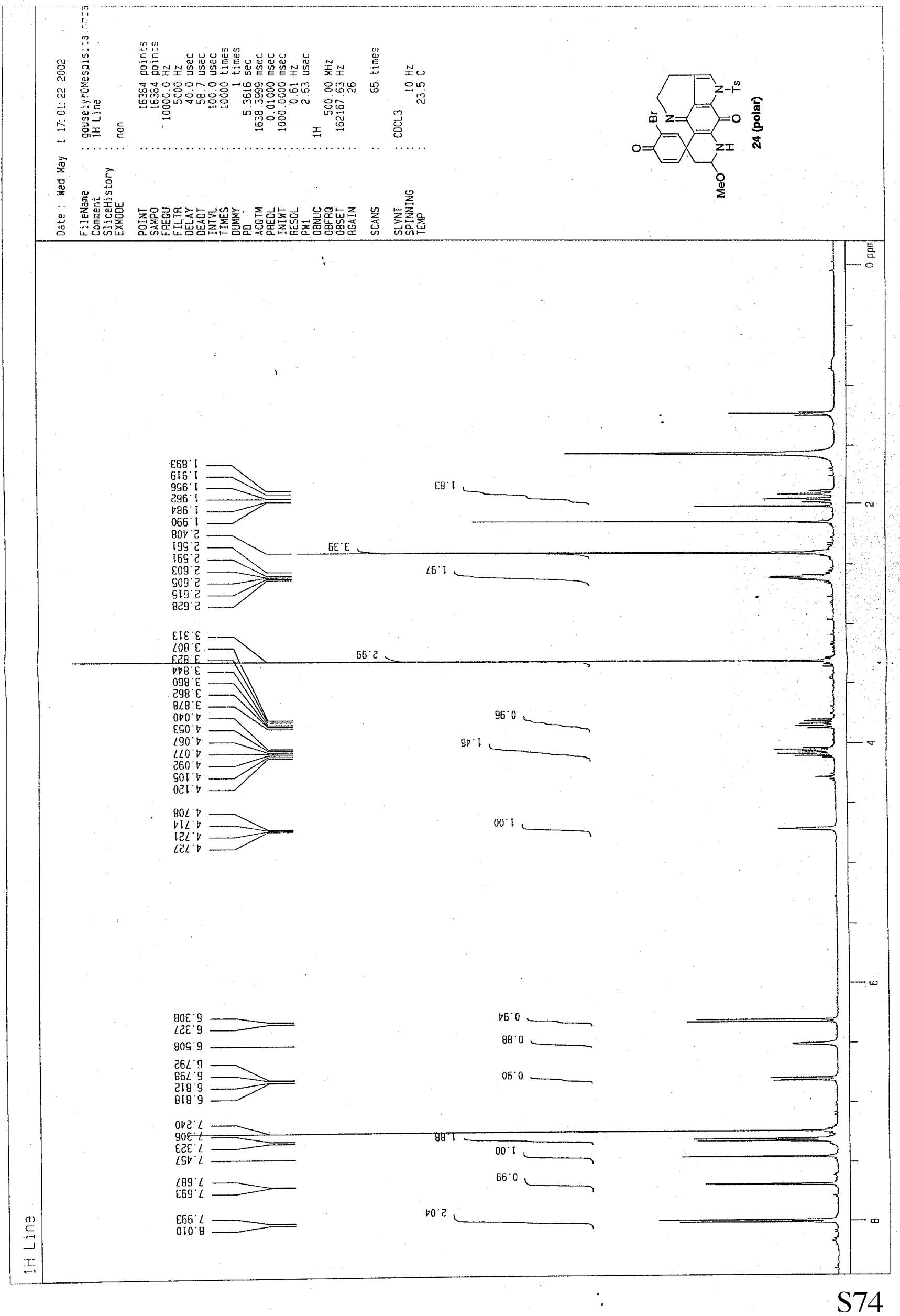




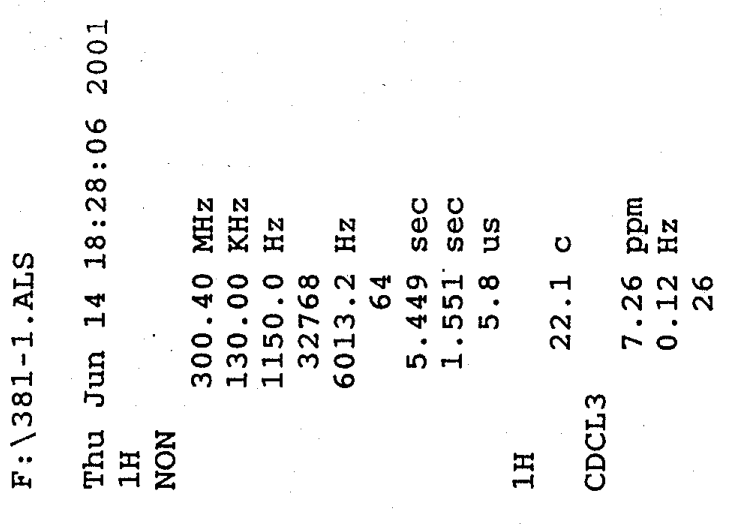

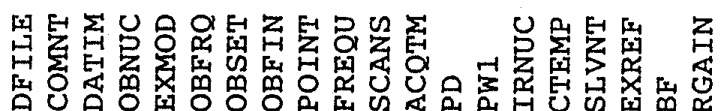
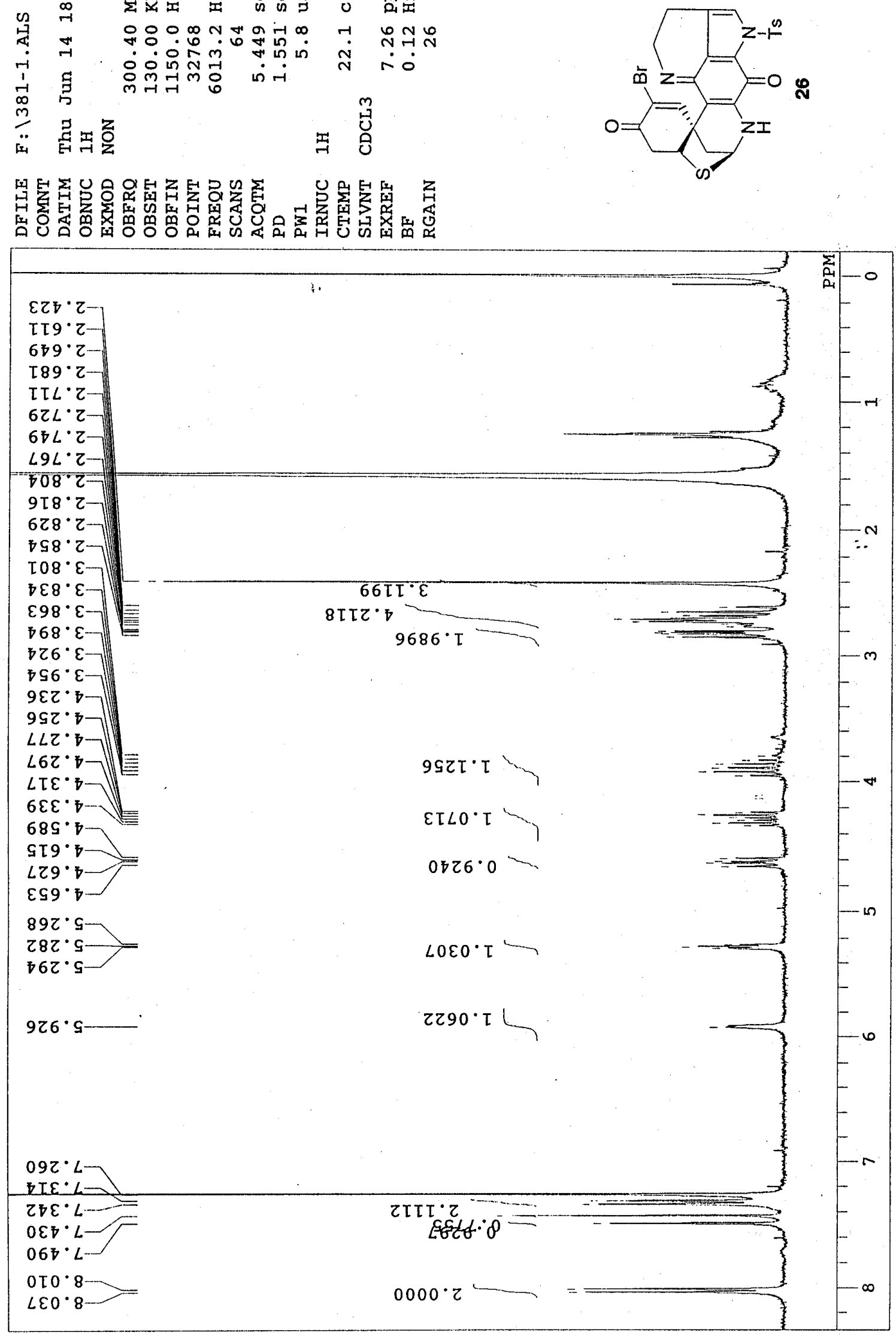

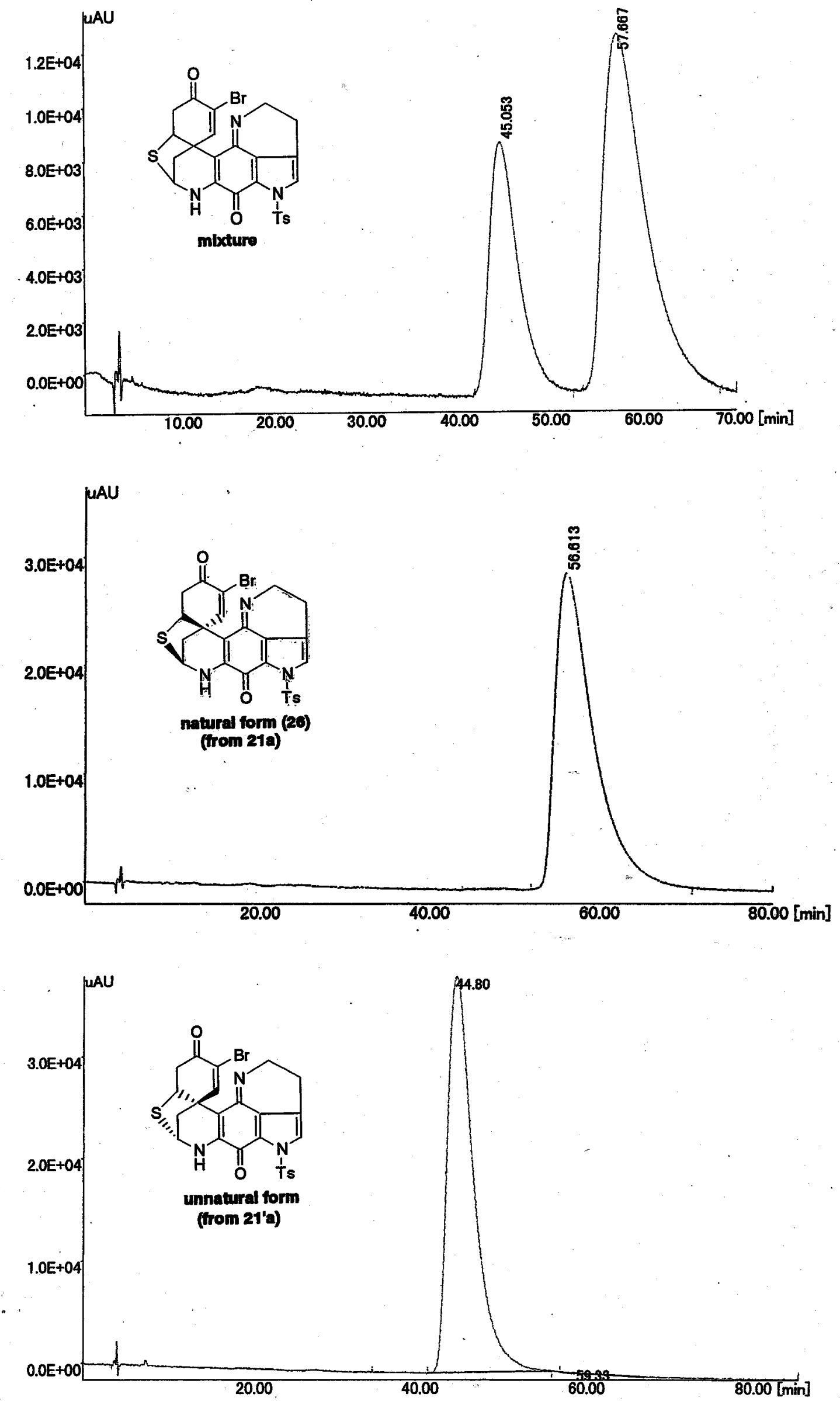

DAICEL CHIRALCEL OD

Hex/PrOH 65/35

$1.0 \mathrm{ml} / \mathrm{min} 20^{\circ} \mathrm{C}$ 
¿ें

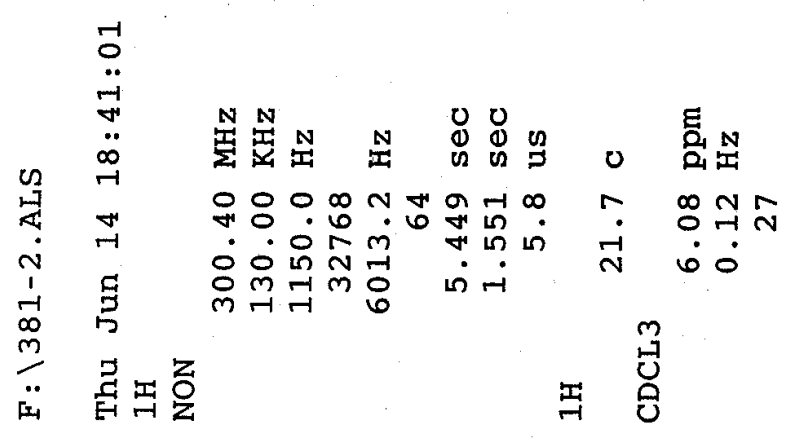

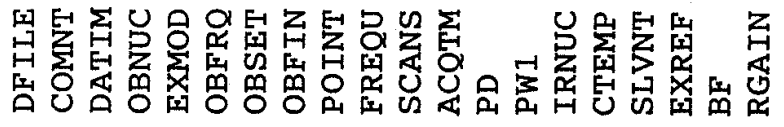

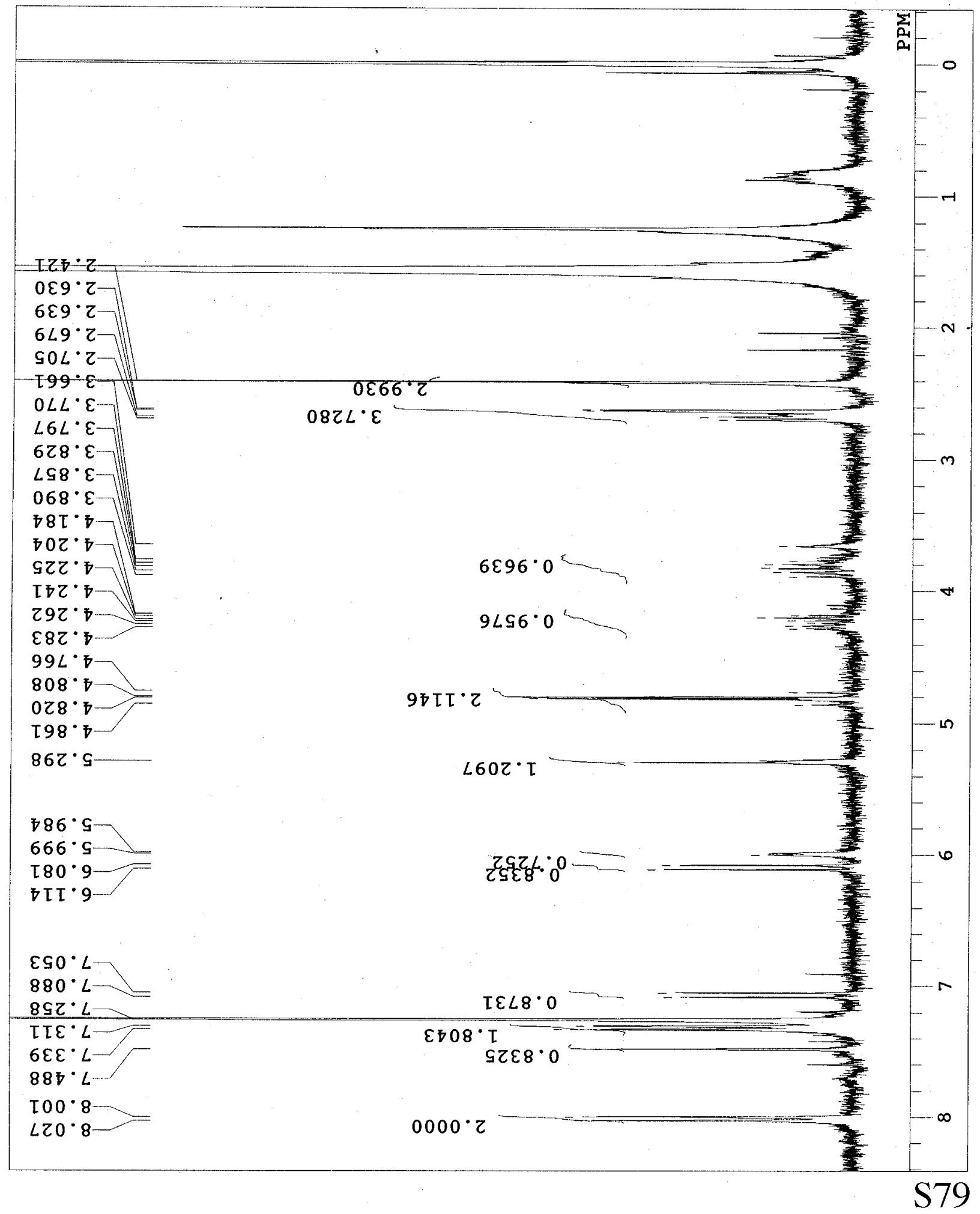




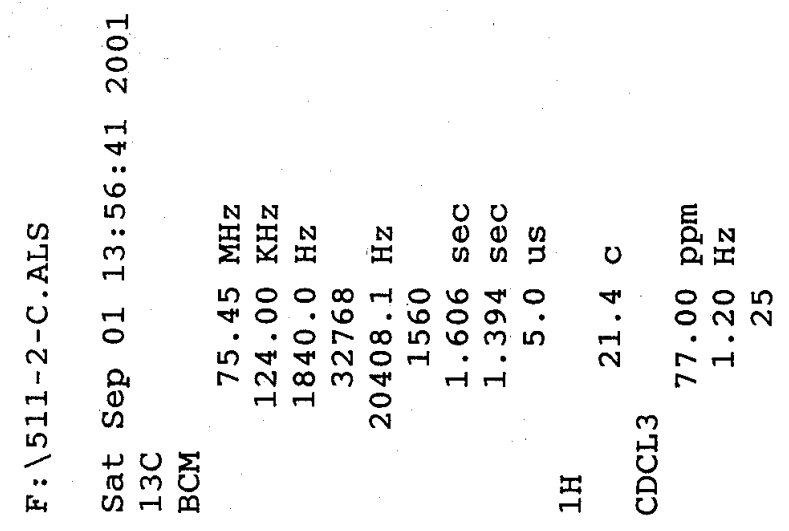

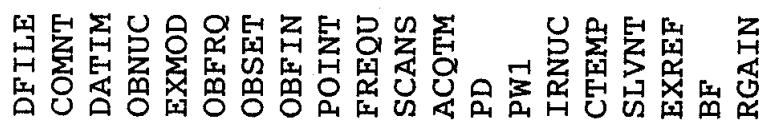
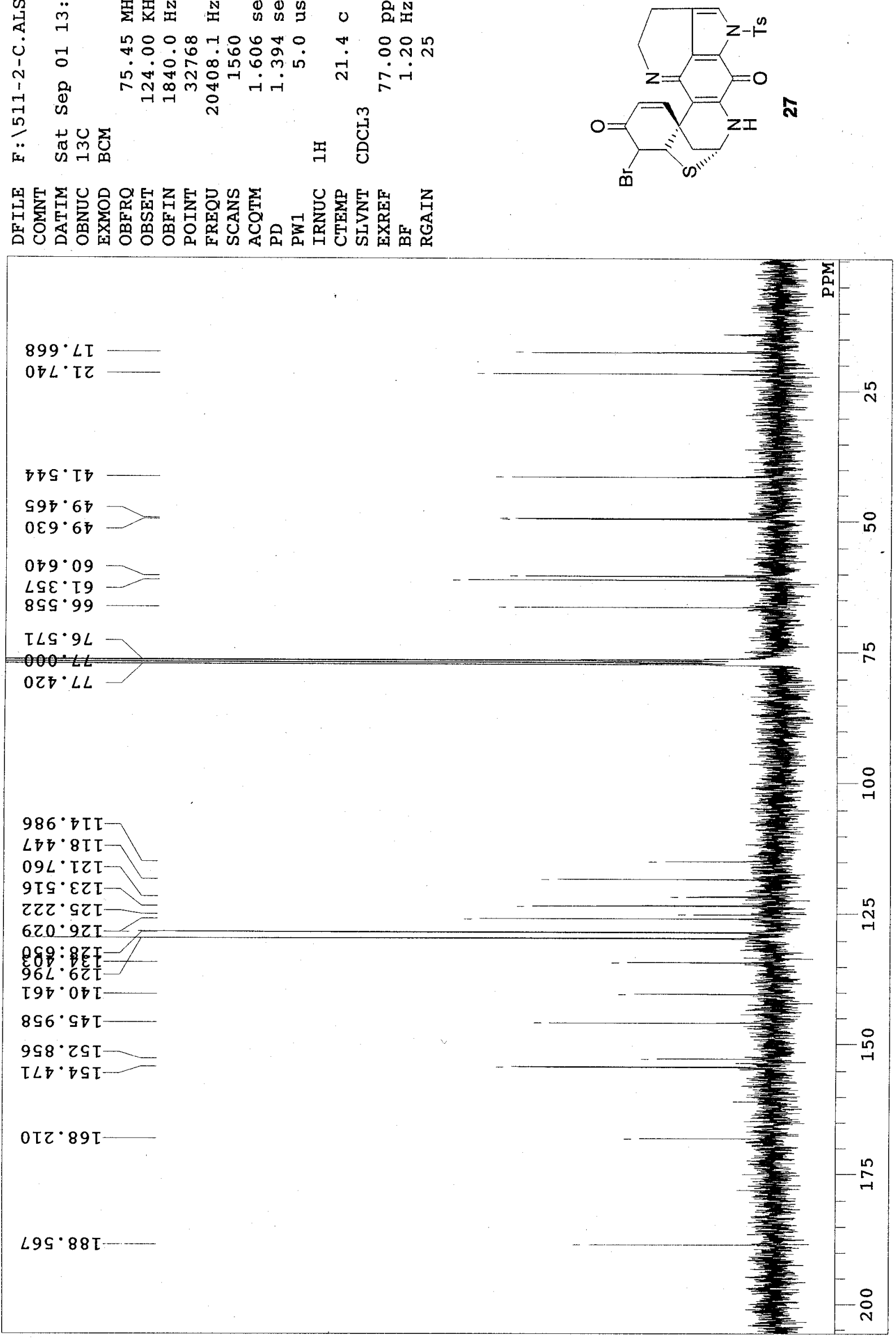


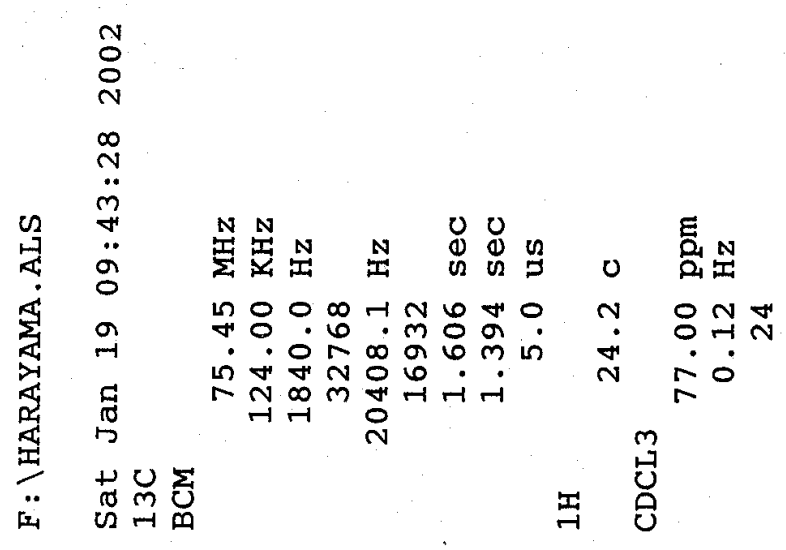

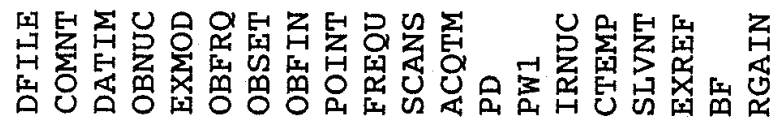
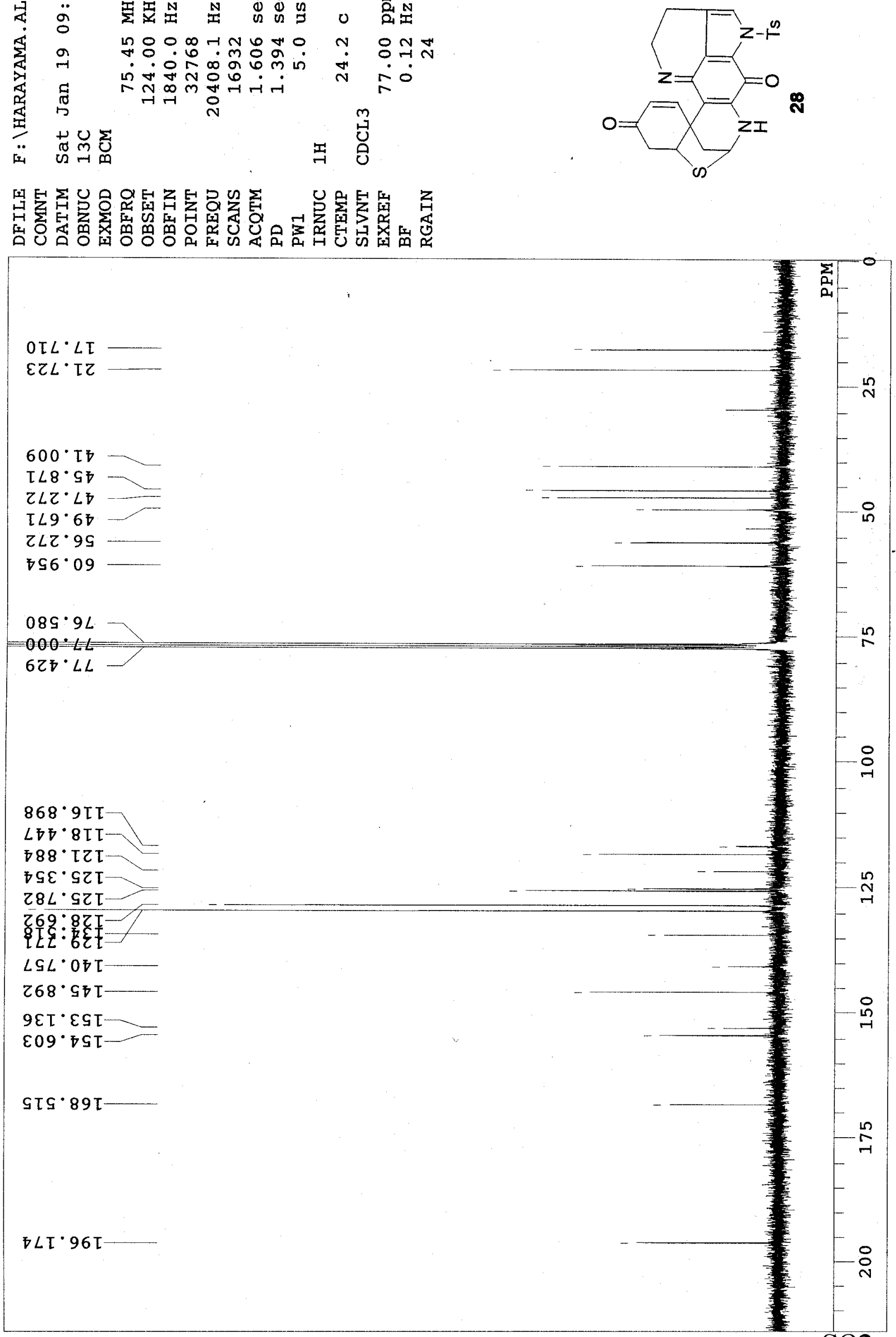

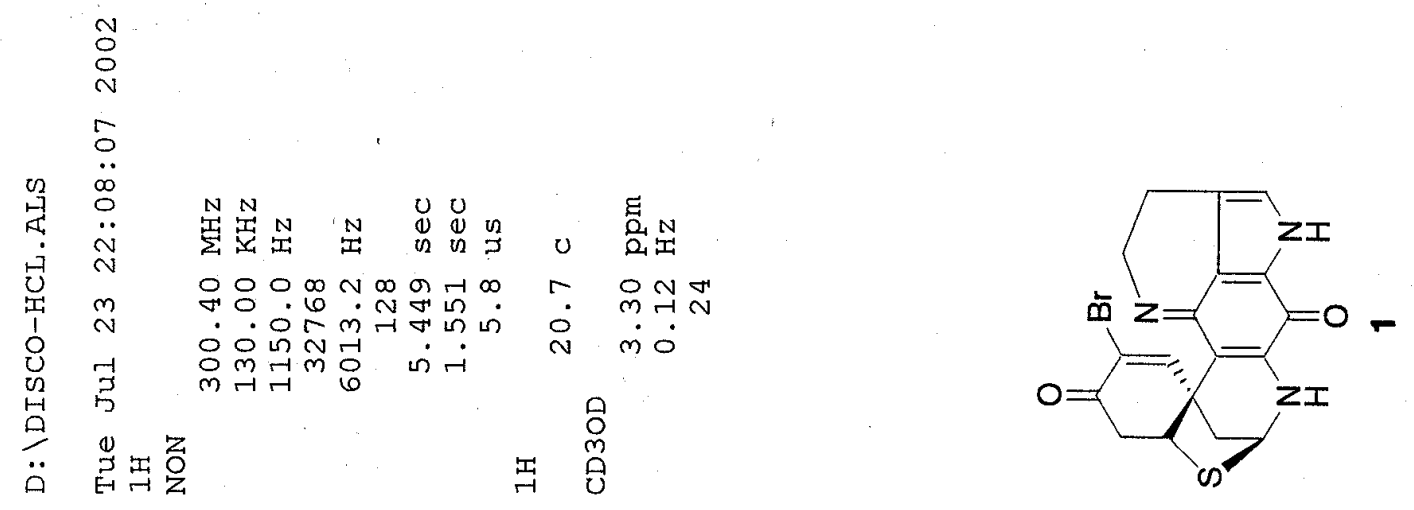

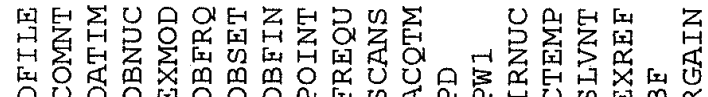

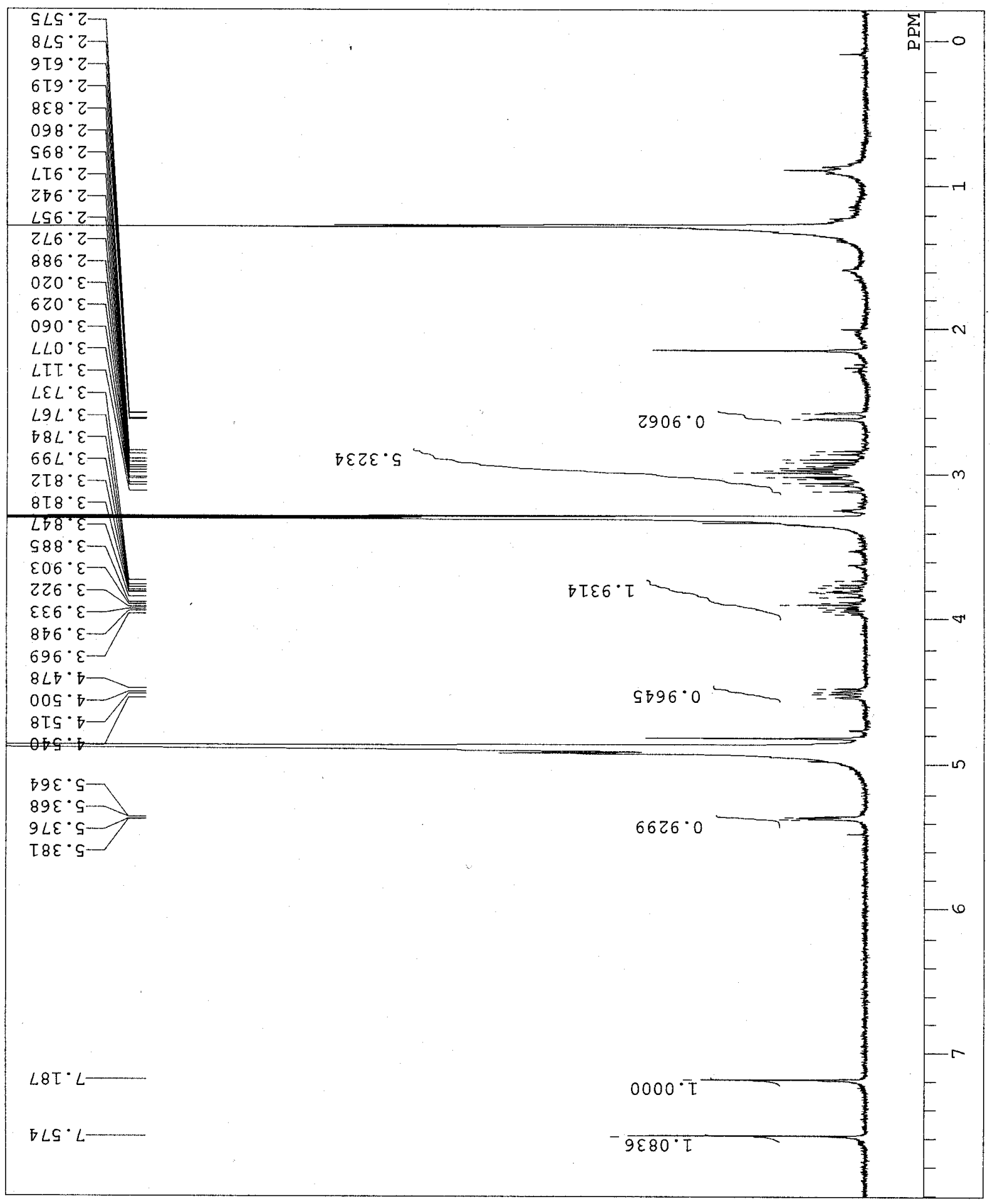




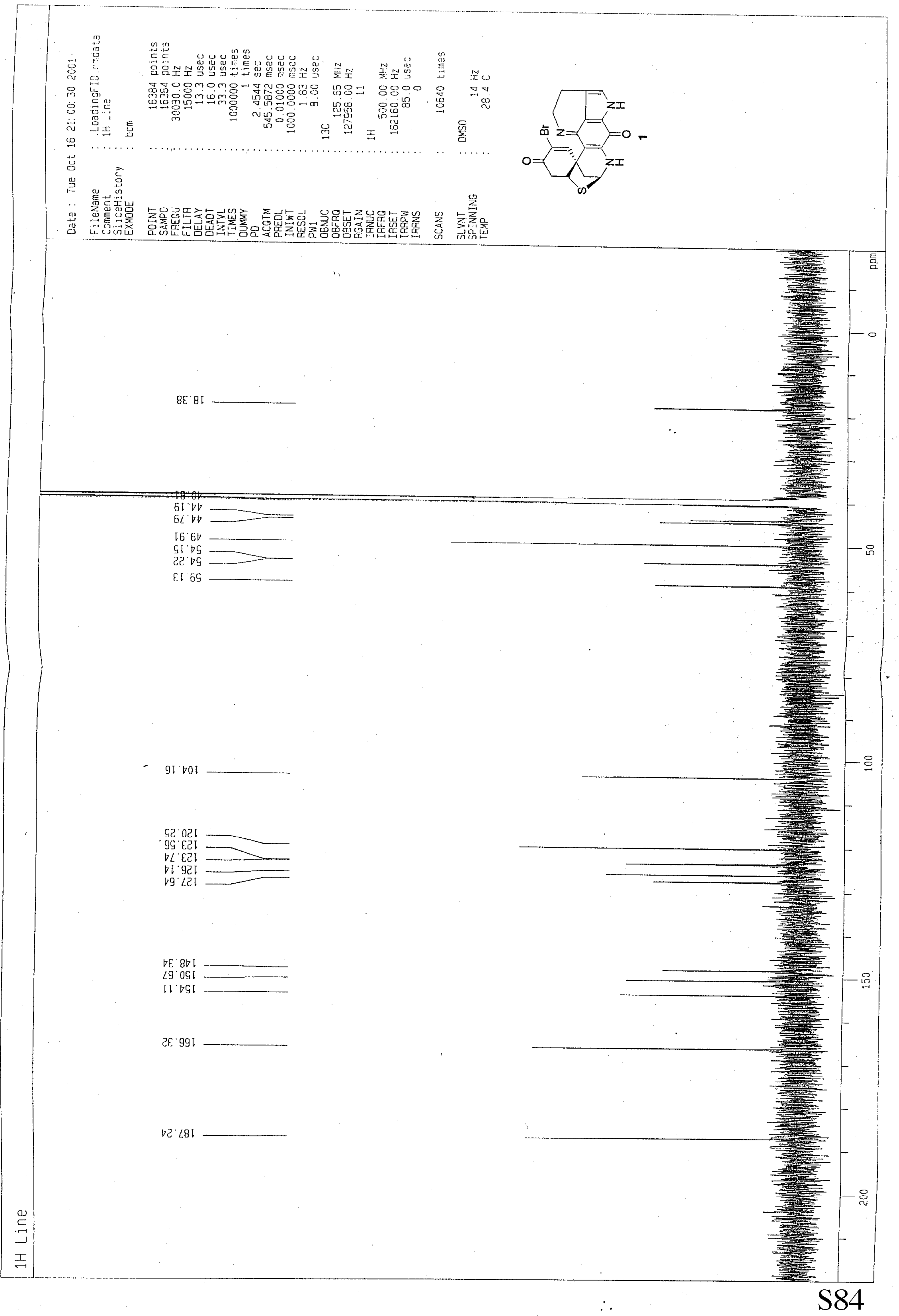




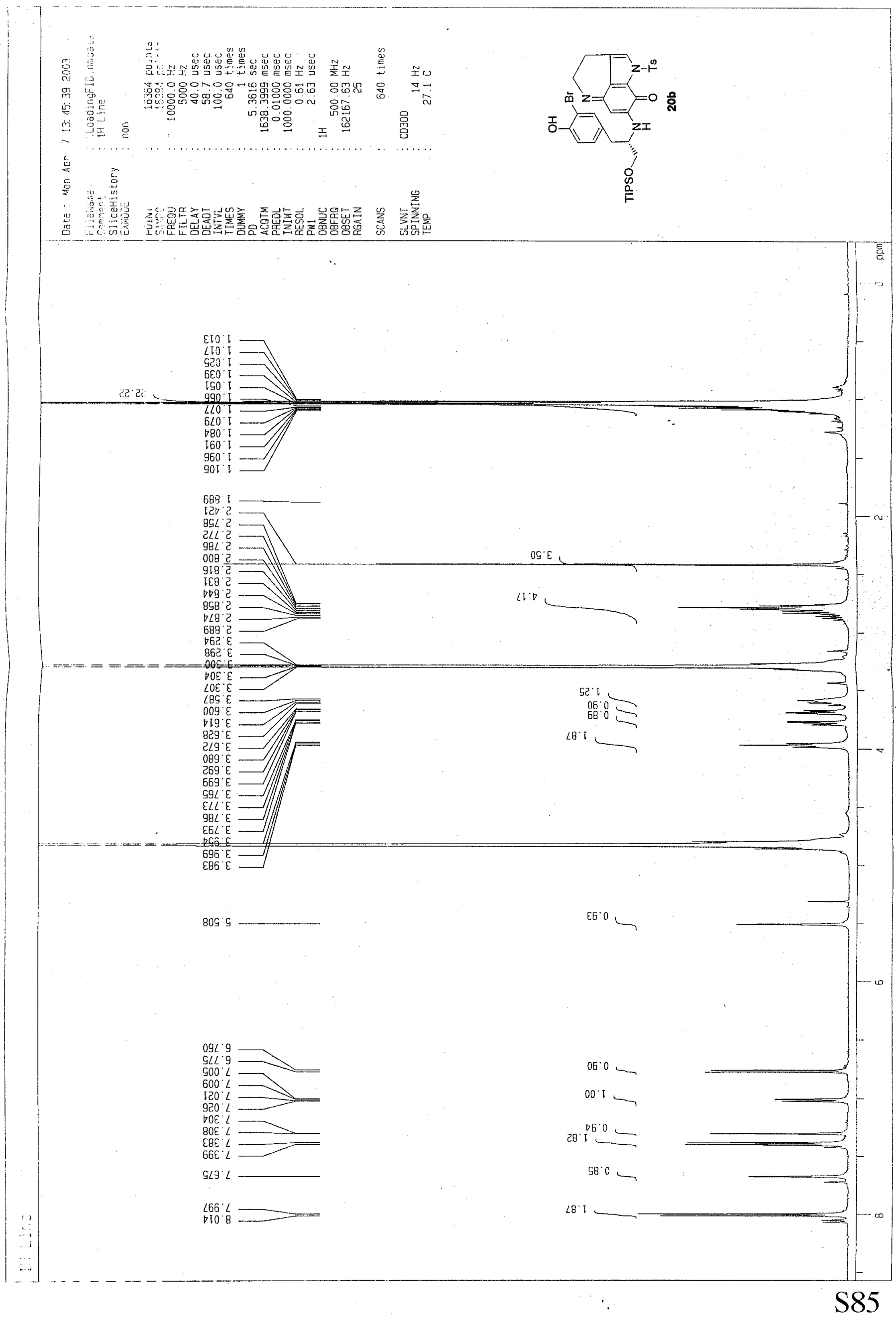




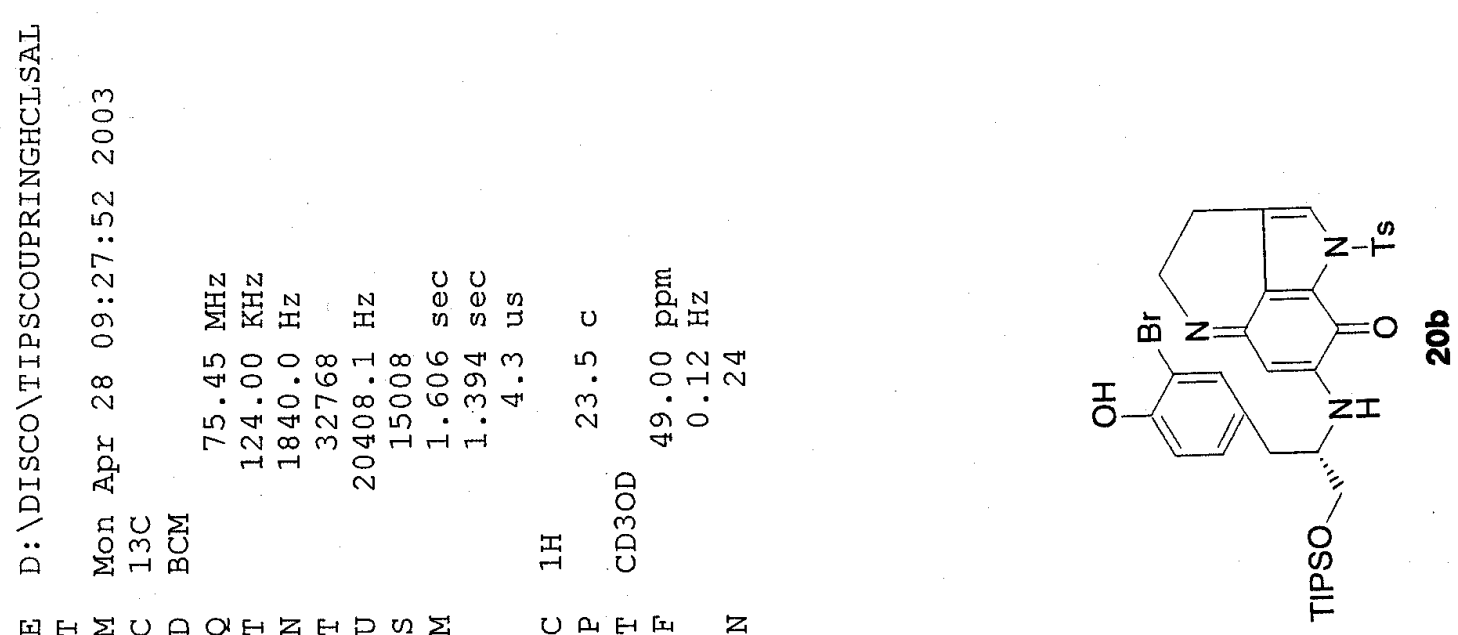

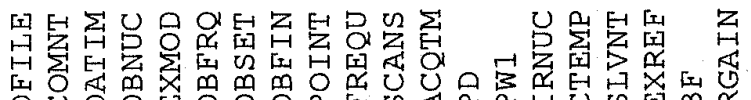

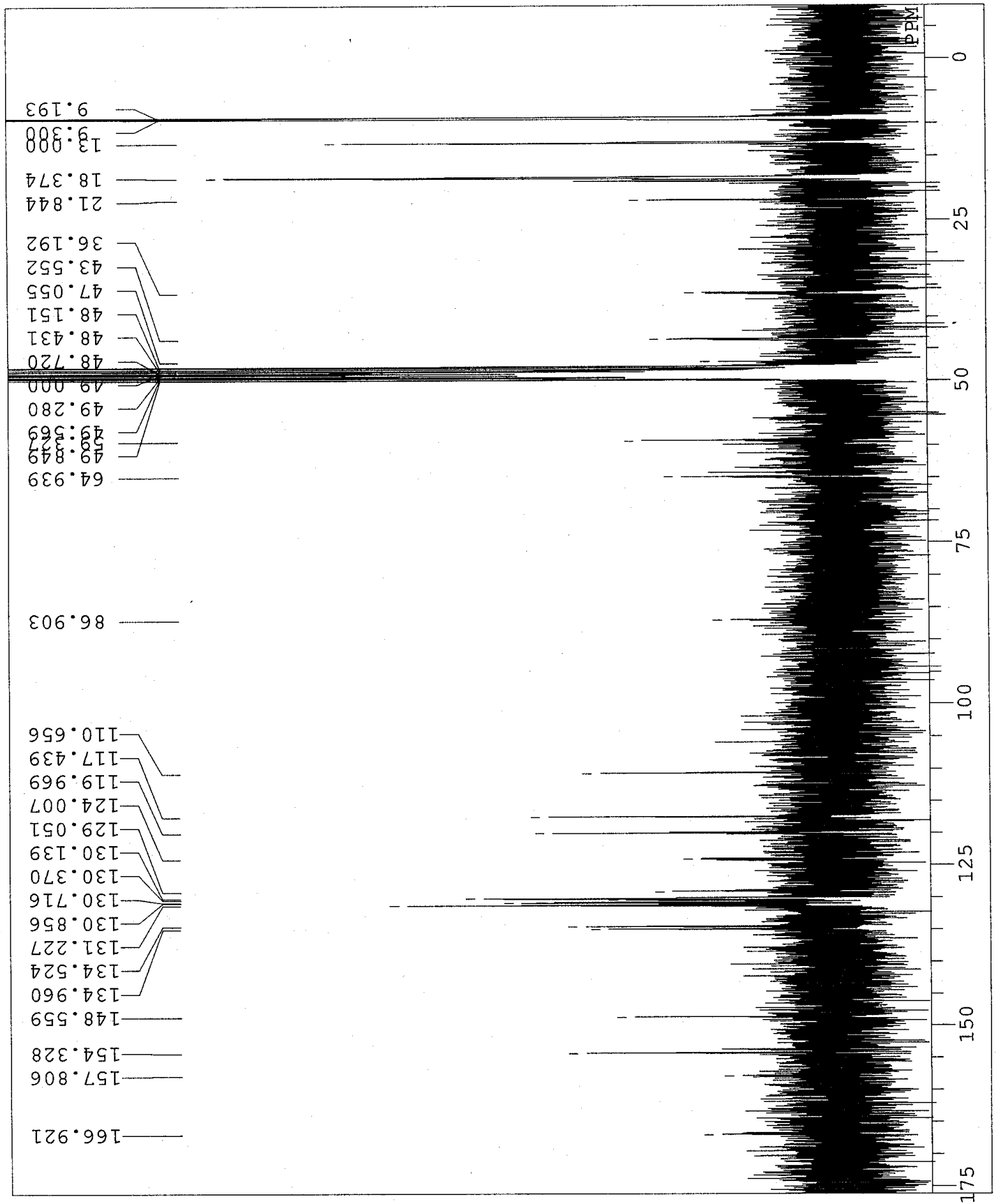




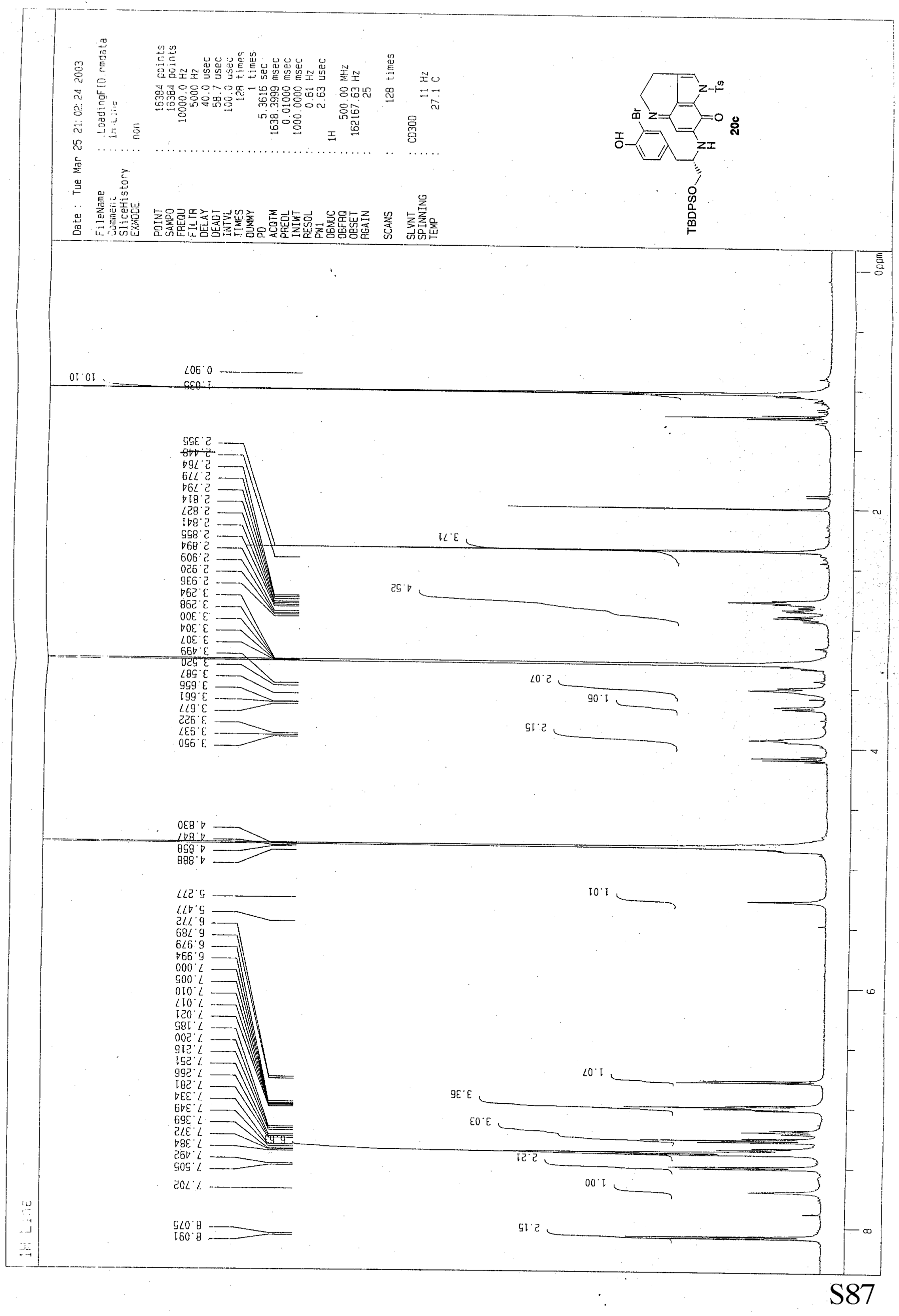




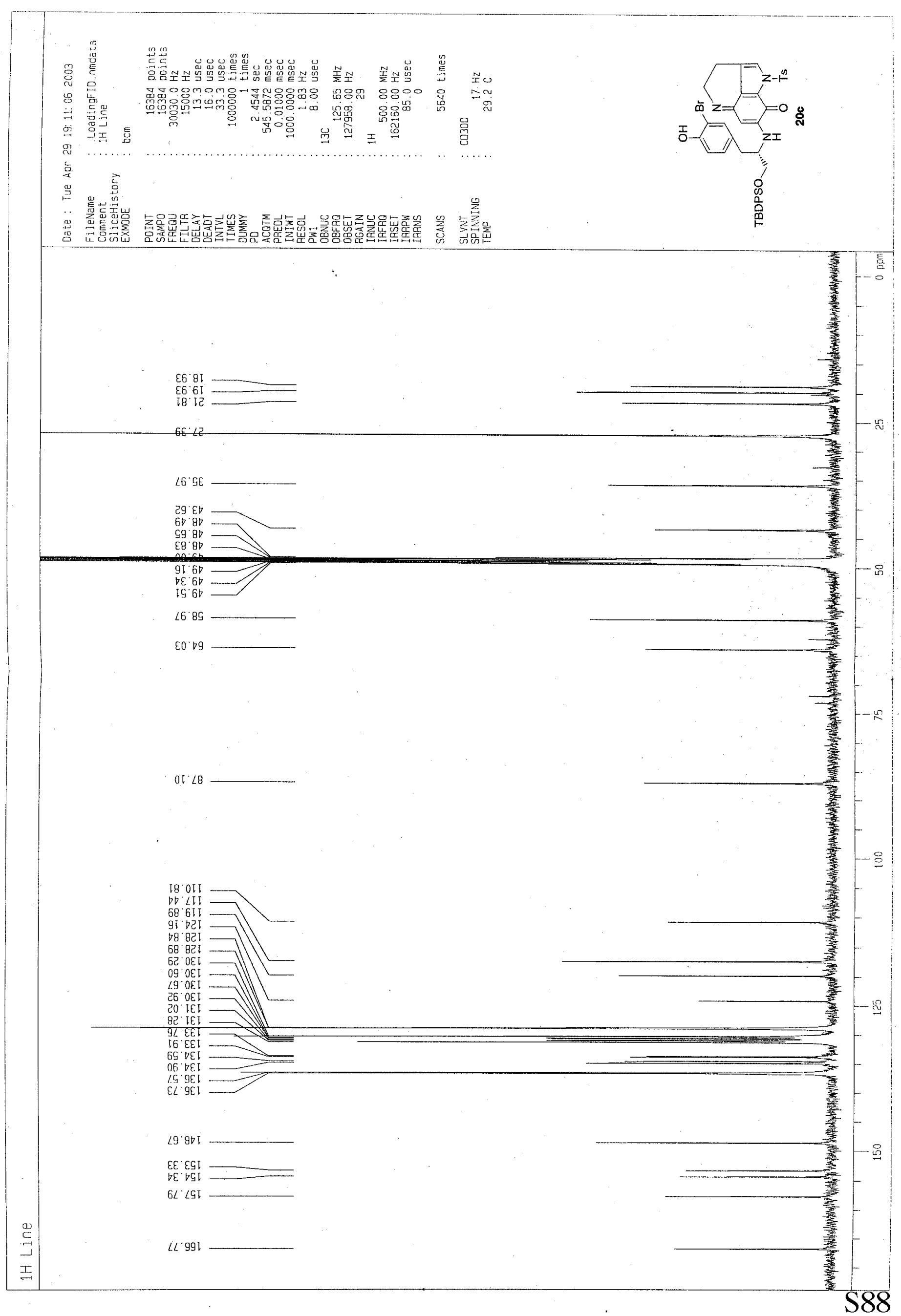




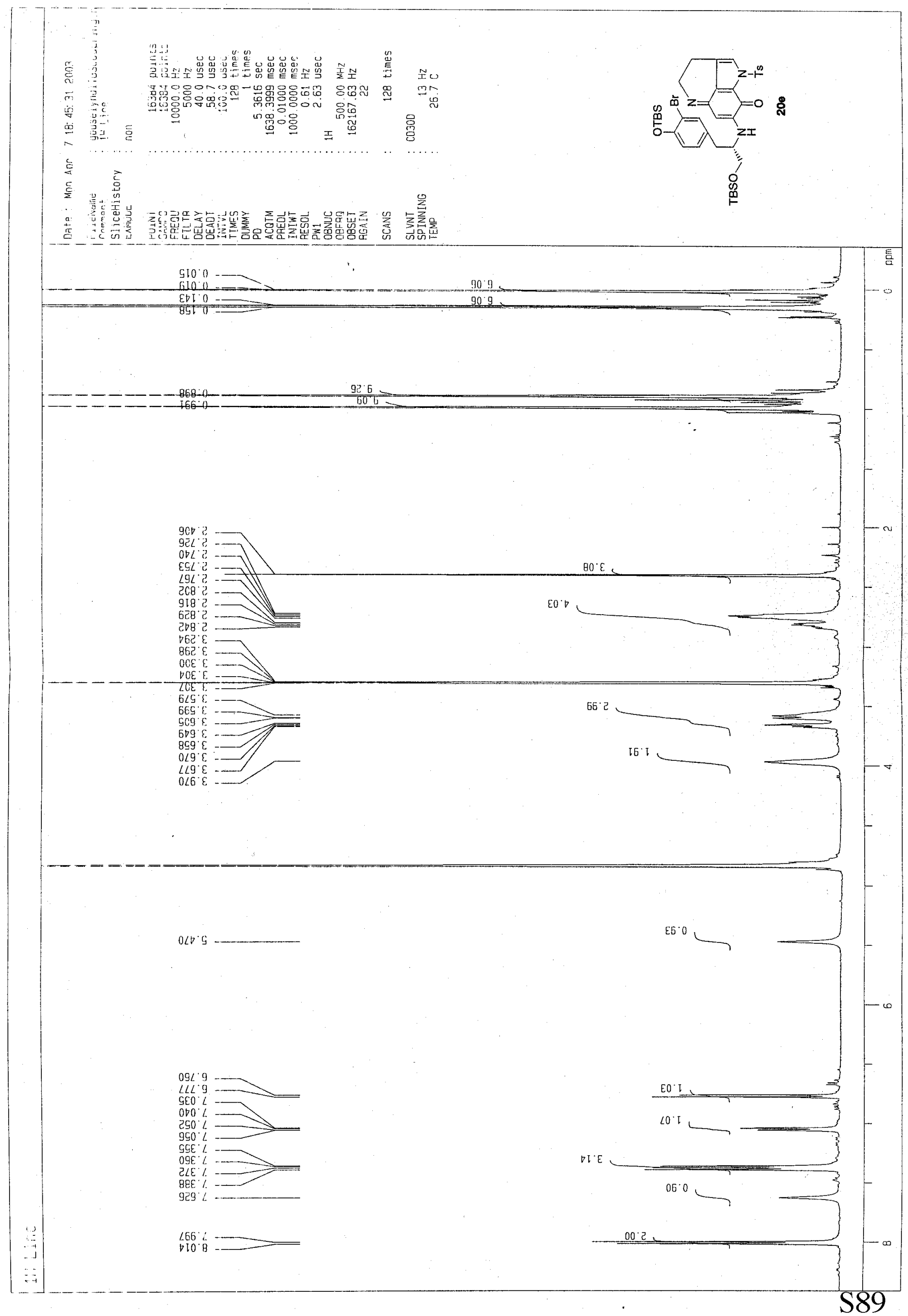




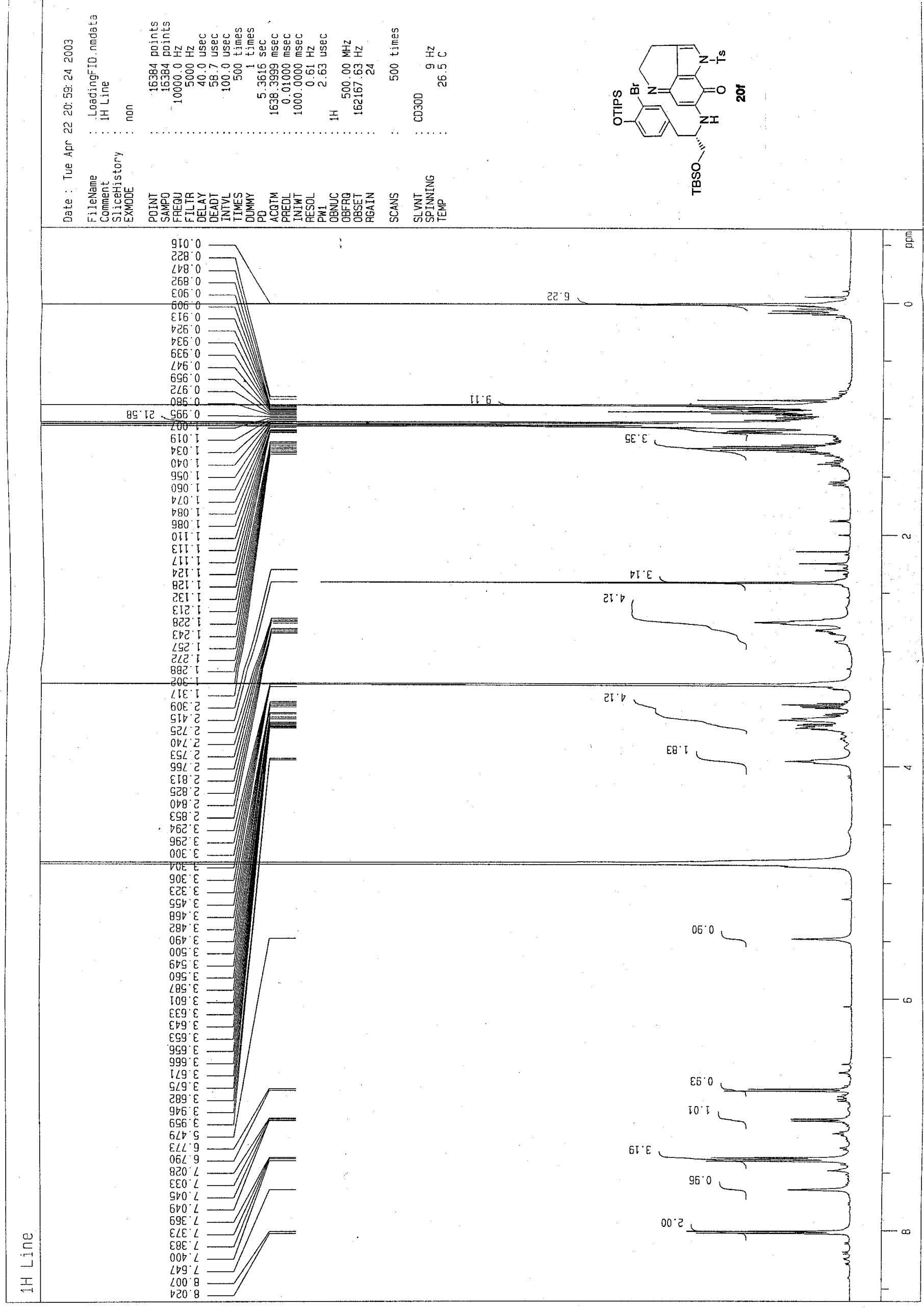




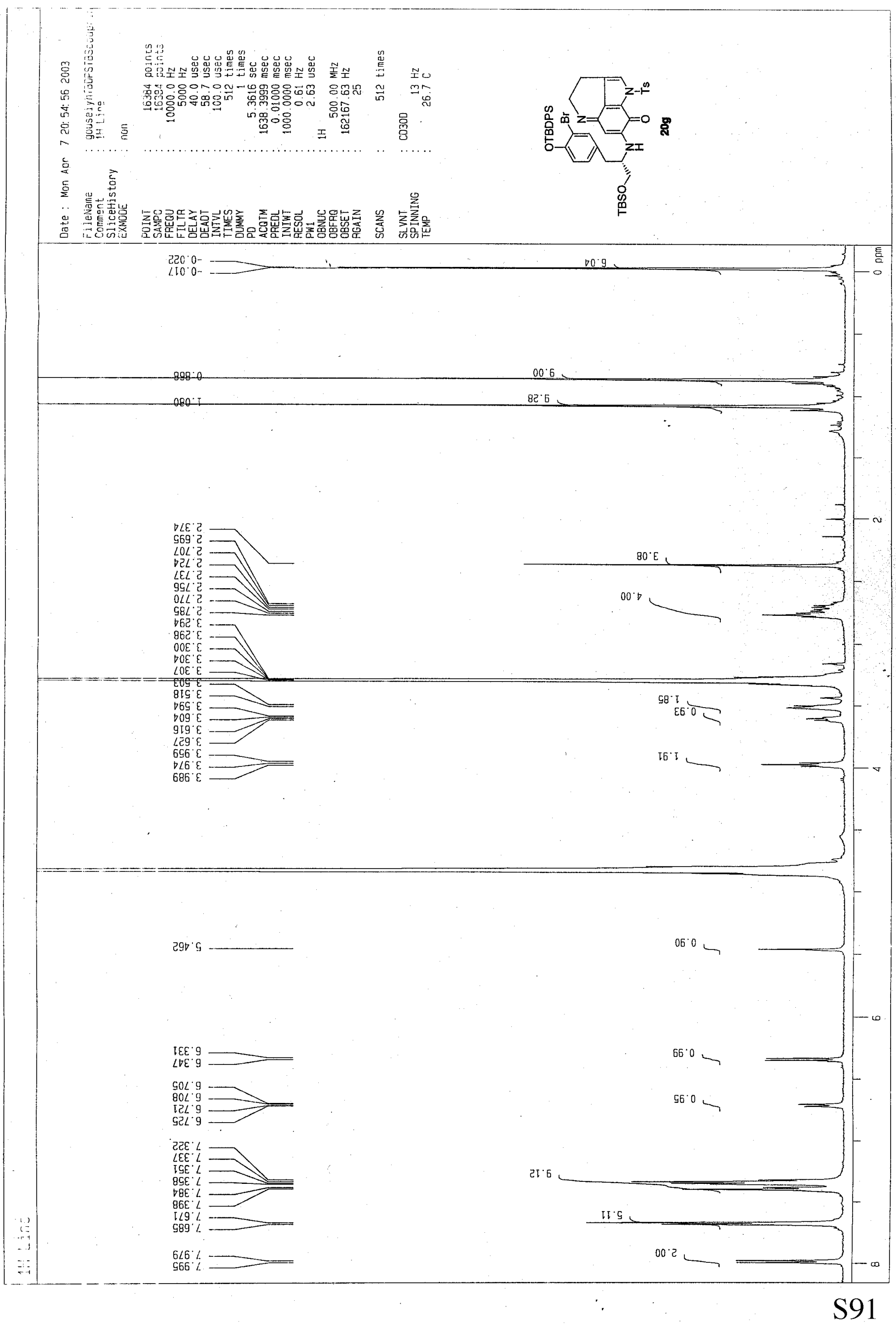




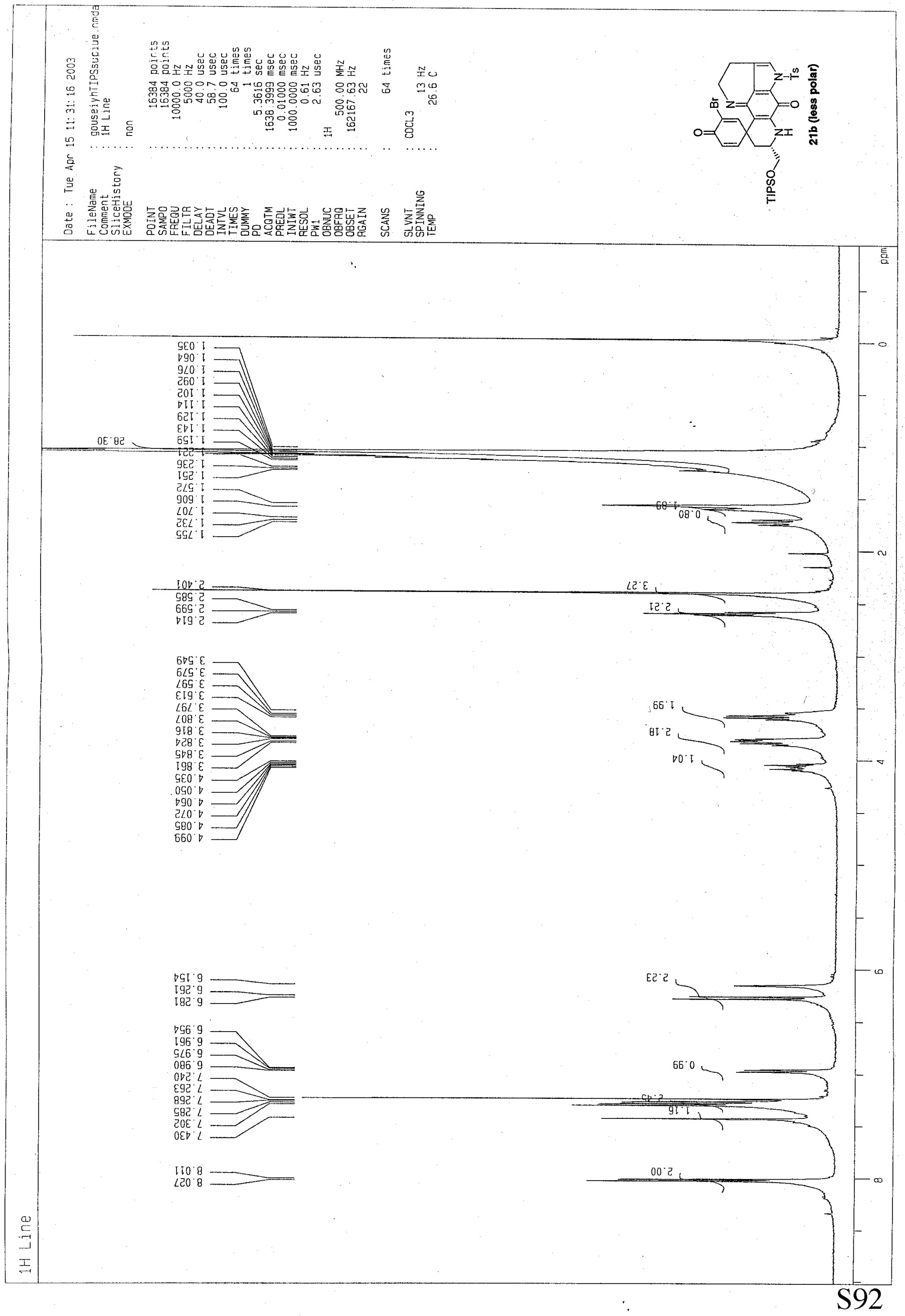




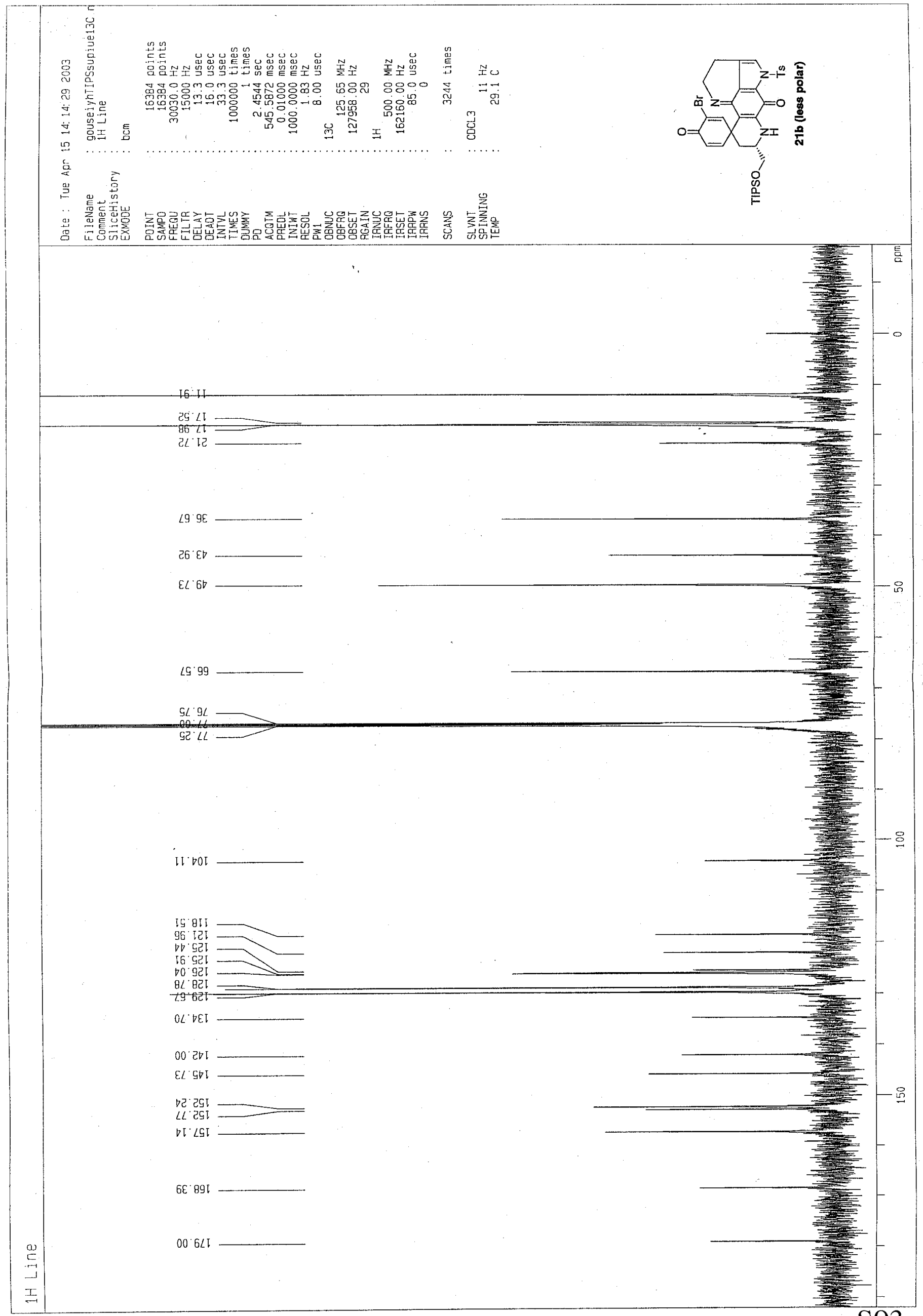




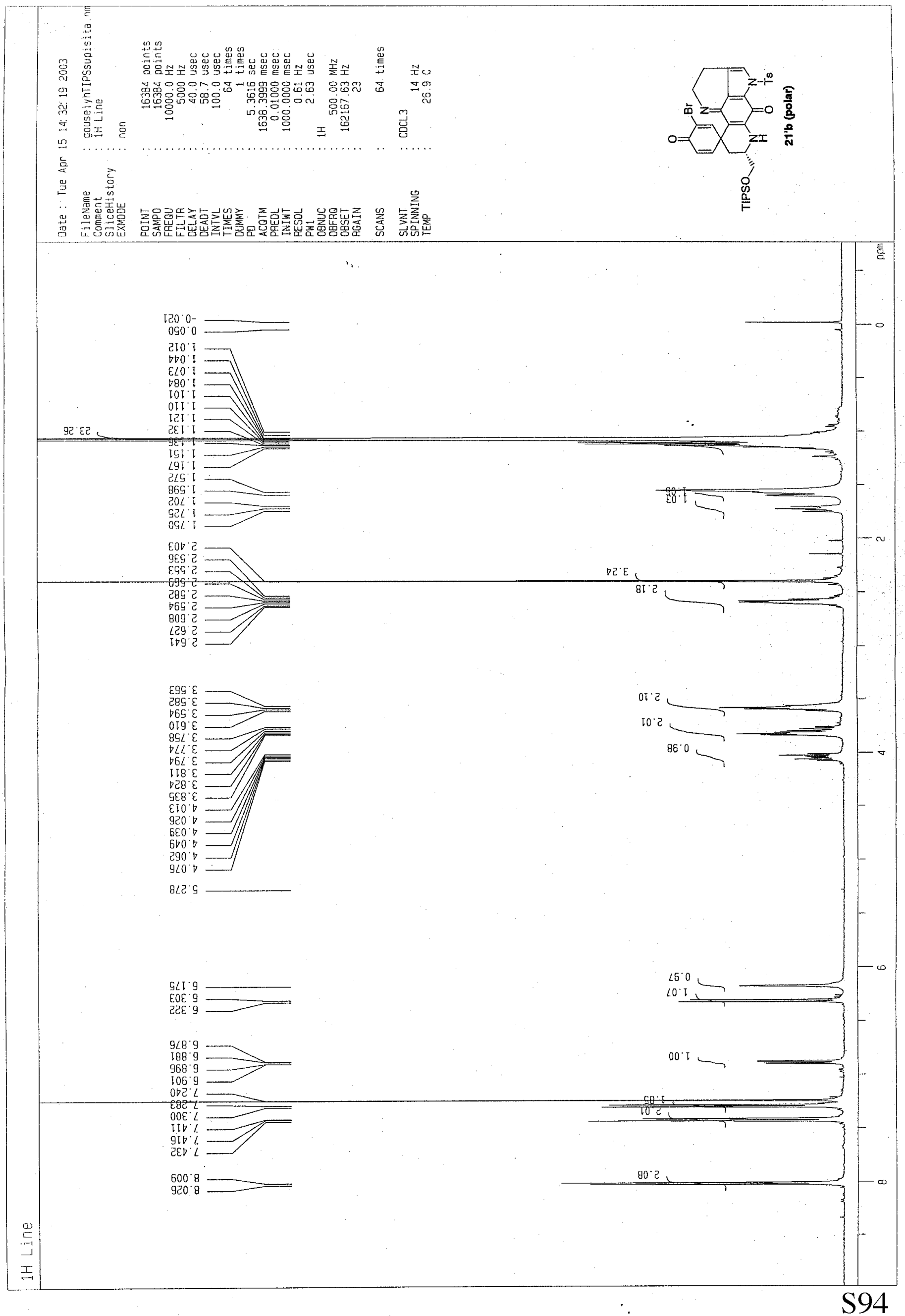




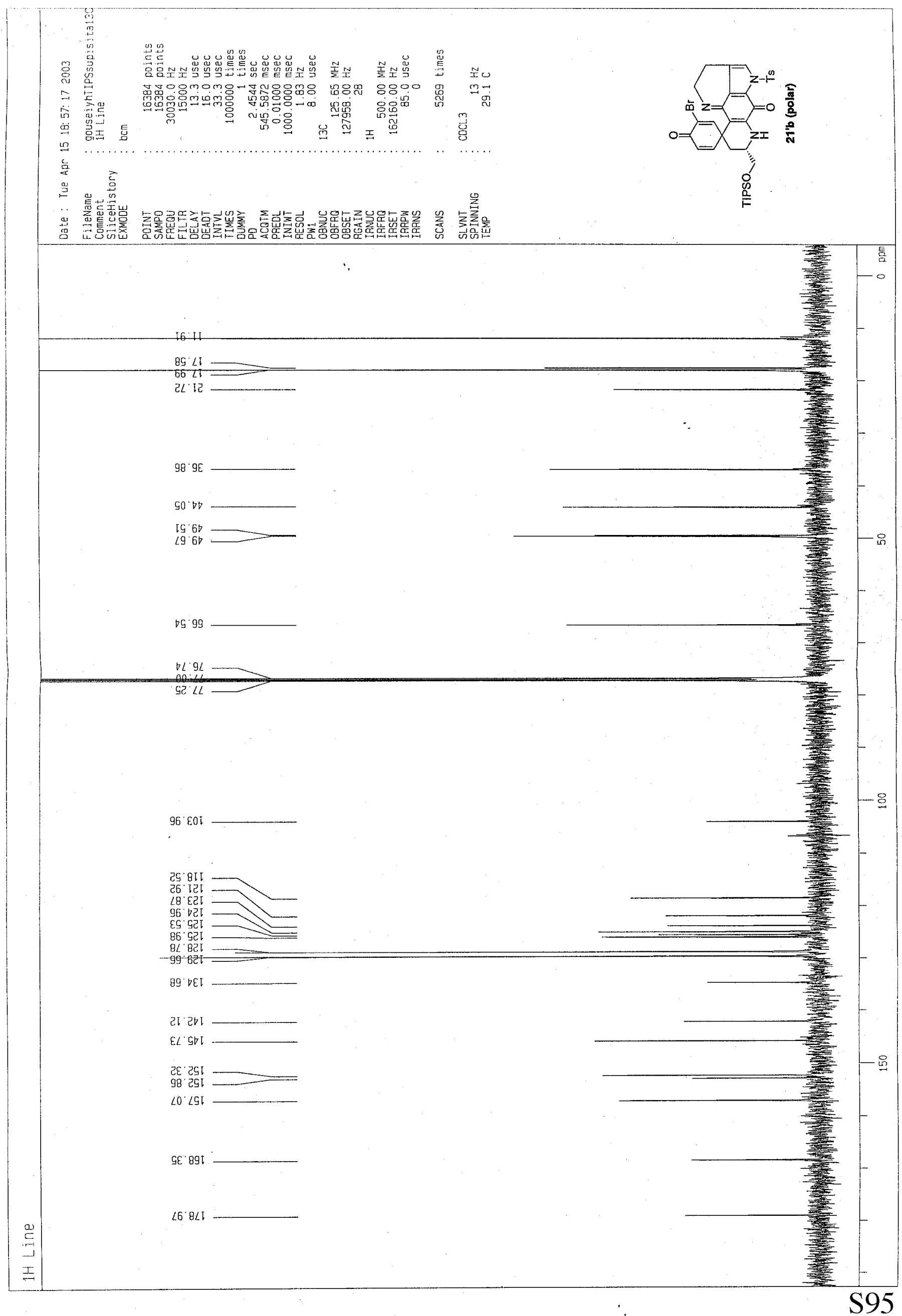




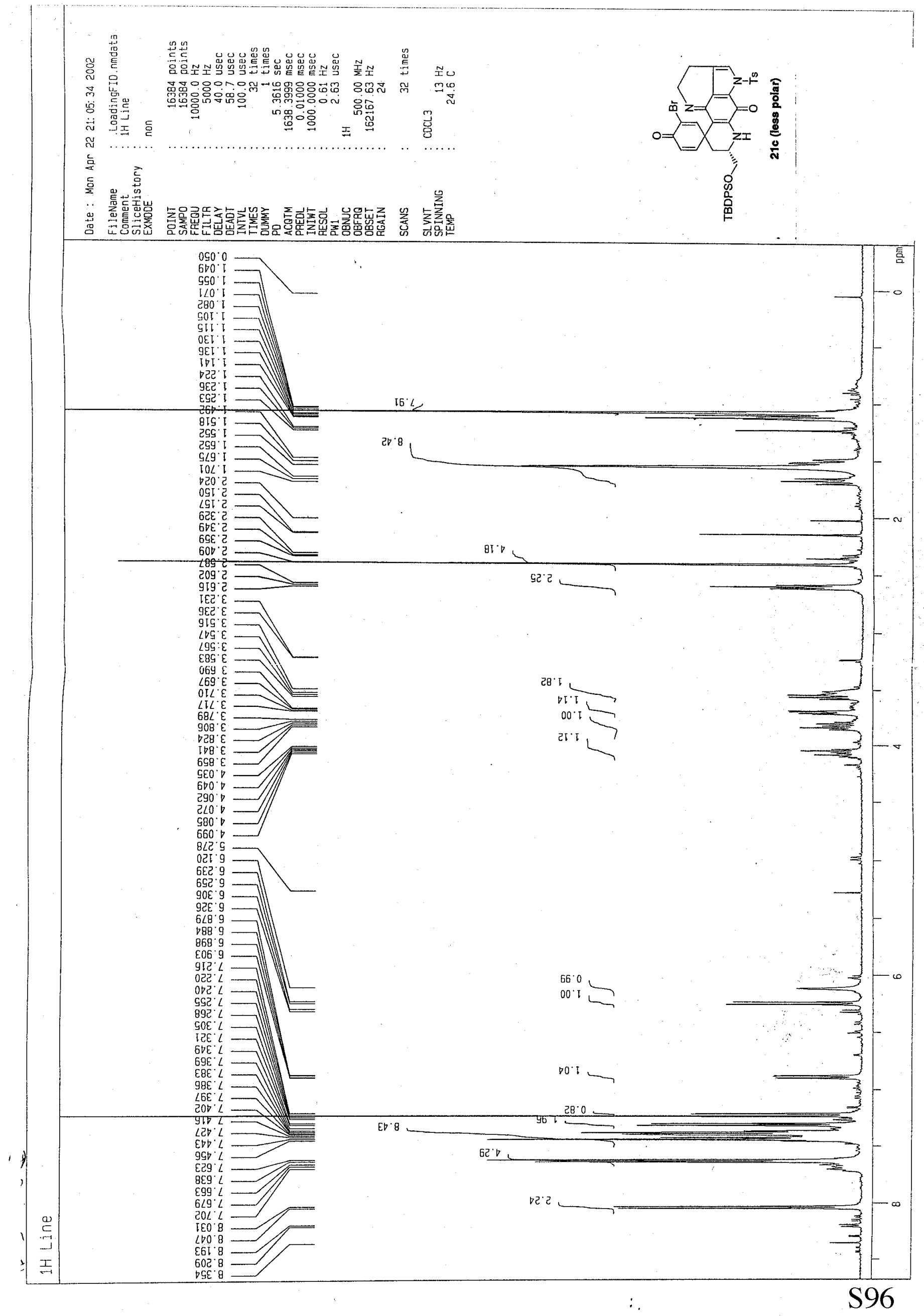




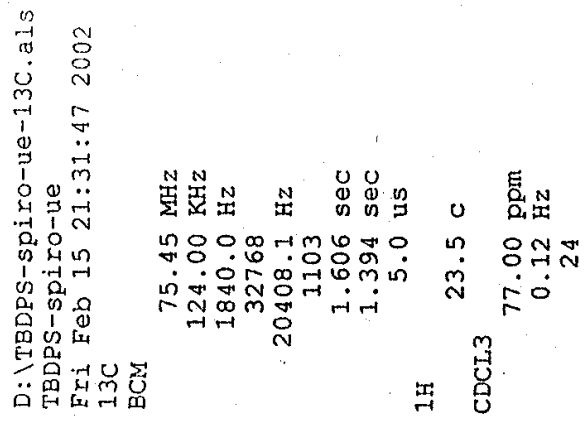

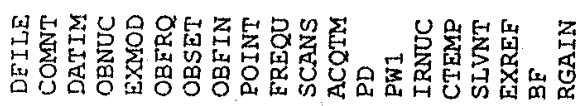

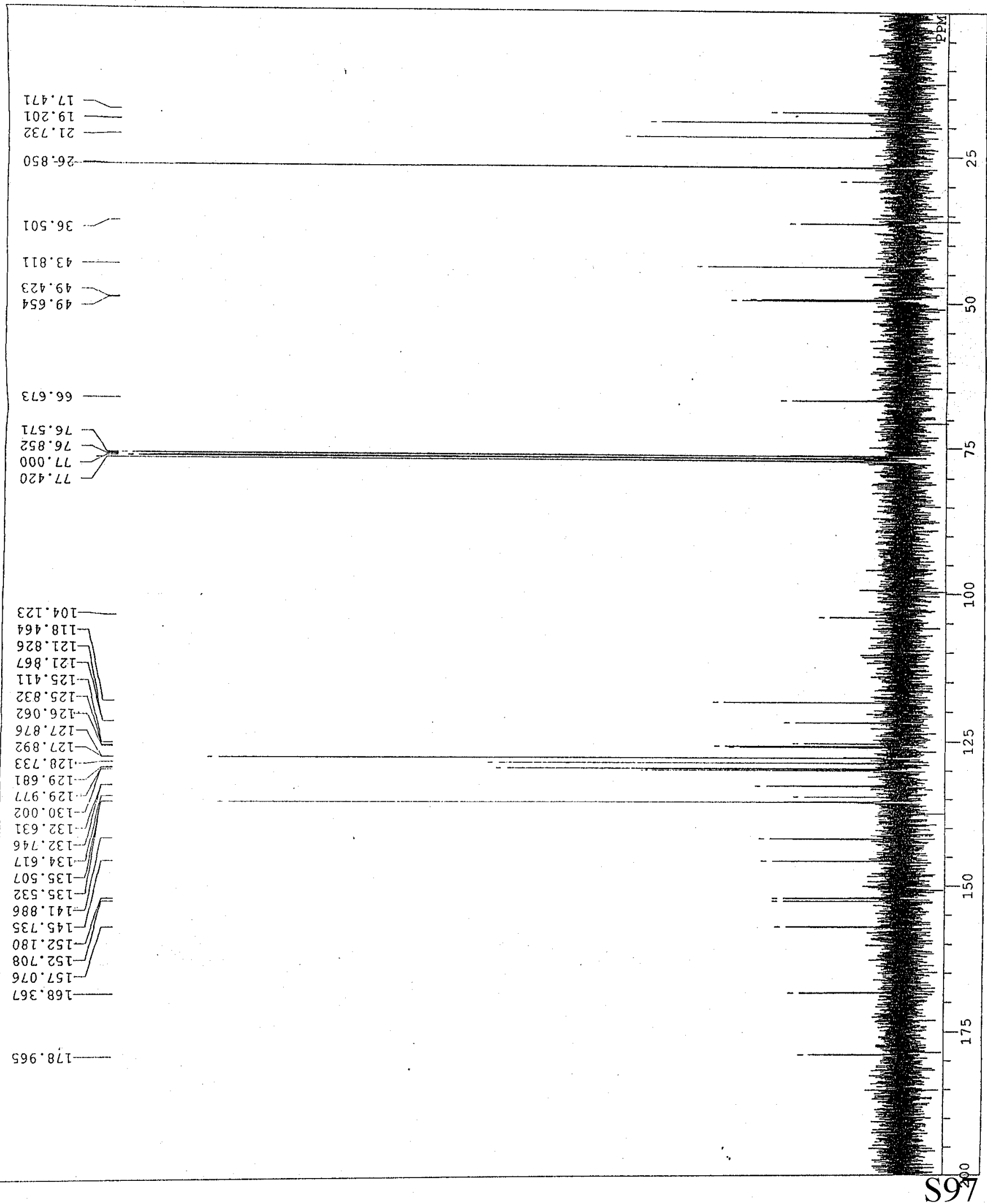




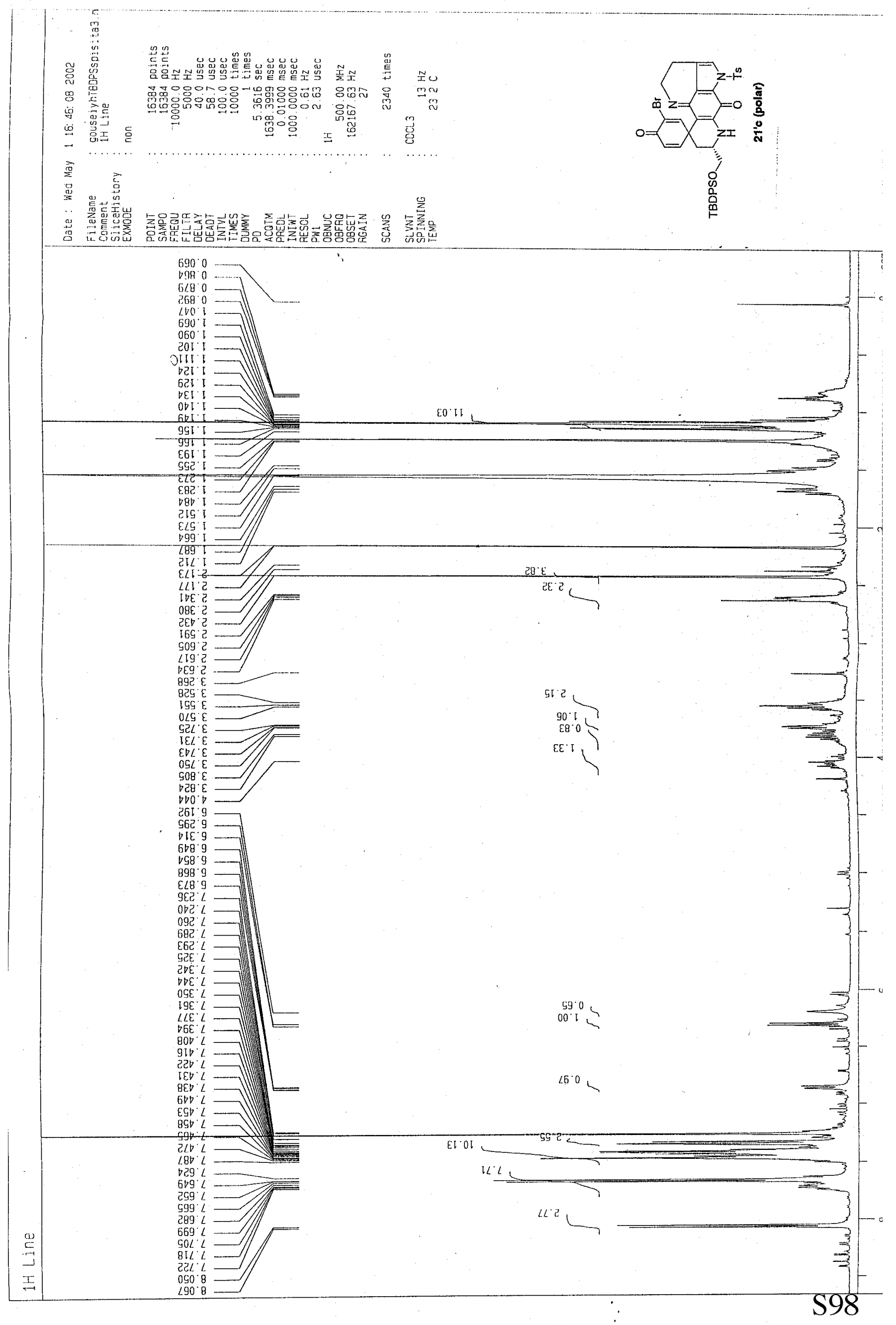




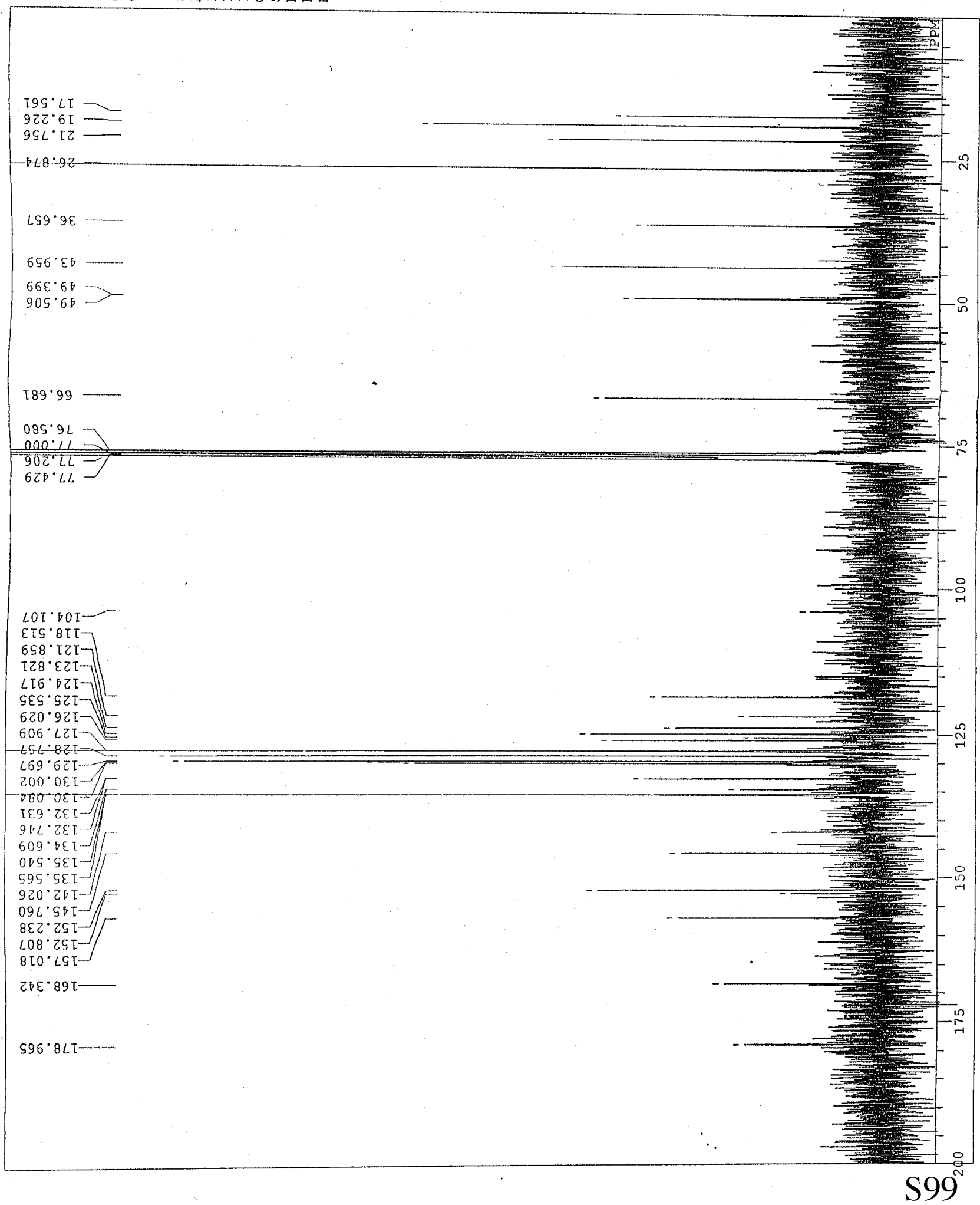

